\title{
Eight salt forms of sulfadiazine
}

\section{Amanda R. Buist, Lynn Dennany, Alan R. Kennedy, Craig Manzie, Katherine McPhie and Brandon Walker}

Acta Cryst. (2014). C70, 900-907

Copyright (C) International Union of Crystallography

Author(s) of this paper may load this reprint on their own web site or institutional repository provided that this cover page is retained. Republication of this article or its storage in electronic databases other than as specified above is not permitted without prior permission in writing from the IUCr.

For further information see http://journals.iucr.org/services/authorrights.html

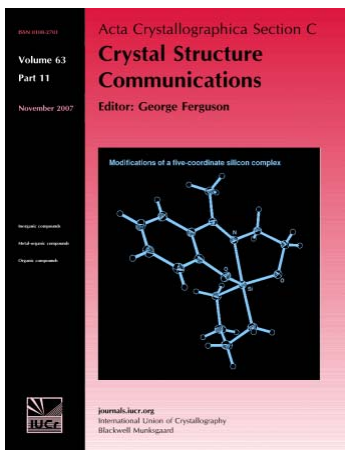

Acta Crystallographica Section C: Structural Chemistry specializes in the rapid dissemination of high-quality detailed studies of novel and challenging crystal and molecular structures of interest in the fields of chemistry, biochemistry, mineralogy, pharmacology, physics and materials science. The unique checking, editing and publishing facilities of the journal ensure the highest standards of structural reliability and presentation, while providing for reports on studies involving special techniques or difficult crystalline materials. Papers go beyond reporting the principal numerical and geometrical data, and may include the discussion of multiple related structures, a detailed description of non-routine structure determinations, placing the structure in an interesting scientific, physical or chemical context, or the discussion of interesting physical properties or modes of association. Reports of difficult or challenging structures, such as cases of twinning, severe disorder, or diffuse solvent regions are welcomed, provided the presented structures are correct and the difficulties and strategies used to treat them are scientifically discussed and properly documented. Section $C$ readers have access to an extensive back archive of high-quality structural data.

\section{Crystallography Journals Online is available from journals.iucr.org}


Acta Crystallographica Section C

\section{Structural Chemistry}

ISSN 2053-2296

\section{Eight salt forms of sulfadiazine}

\section{Amanda R. Buist,* Lynn Dennany, Alan R. Kennedy,* Craig Manzie, Katherine McPhie and Brandon Walker}

Westchem, Department of Pure \& Applied Chemistry, University of Strathclyde, 295

Cathedral Street, Glasgow G1 1XL, Scotland

Correspondence e-mail: amanda.buist.2013@uni.strath.ac.uk,

a.r.kennedy@strath.ac.uk

Received 4 August 2014

Accepted 18 August 2014

Proton transfer to the sulfa drug sulfadiazine [systematic name: 4-amino- $N$-(pyrimidin-2-yl)benzenesulfonamide] gave eight salt forms. These are the monohydrate and methanol hemisolvate forms of the chloride (2-\{[(4-azaniumylphenyl)sulfonyl]azanidyl $\}$ pyrimidin-1-ium chloride monohydrate, $\mathrm{C}_{10} \mathrm{H}_{11} \mathrm{~N}_{4} \mathrm{O}_{2} \mathrm{~S}^{+} \cdot \mathrm{Cl}^{-} \cdot \mathrm{H}_{2} \mathrm{O}$, (I), and 2-\{[(4-azaniumylphenyl)sulfonyl]azanidyl pyrimidin-1-ium chloride methanol hemisolvate, $\mathrm{C}_{10} \mathrm{H}_{11} \mathrm{~N}_{4} \mathrm{O}_{2} \mathrm{~S}^{+} \cdot \mathrm{Cl}^{-} \cdot 0.5 \mathrm{CH}_{3} \mathrm{OH}$, (II)); a bromide monohydrate (2-\{[(4-azaniumylphenyl)sulfonyl $]$ azanidyl $\}$ pyrimidin1-ium bromide monohydrate, $\mathrm{C}_{10} \mathrm{H}_{11} \mathrm{~N}_{4} \mathrm{O}_{2} \mathrm{~S}^{+} \cdot \mathrm{Br}^{-} \cdot \mathrm{H}_{2} \mathrm{O}$, (III)), which has a disordered water channel; a species containing the unusual tetraiodide dianion [bis(2-\{[(4-azaniumylphenyl)sulfonyl]azanidyl\}pyrimidin-1-ium) tetraiodide, $2 \mathrm{C}_{10} \mathrm{H}_{11} \mathrm{~N}_{4}$ $\mathrm{O}_{2} \mathrm{~S}^{+} \cdot \mathrm{I}_{4}{ }^{2-}$, (IV)], where the $\left[\mathrm{I}_{4}\right]^{2-}$ ion is located at a crystallographic inversion centre; a tetrafluoroborate monohydrate (2-\{[(4-azaniumylphenyl)sulfonyl $]$ azanidyl $\}$ pyrimidin1-ium tetrafluoroborate monohydrate, $\mathrm{C}_{10} \mathrm{H}_{11} \mathrm{~N}_{4} \mathrm{O}_{2} \mathrm{~S}^{+} \cdot \mathrm{BF}_{4}{ }^{-}$.$\left.\mathrm{H}_{2} \mathrm{O},(\mathrm{V})\right)$; a nitrate (2-\{[(4-azaniumylphenyl)sulfonyl $]$ azanidyl)pyrimidin-1-ium nitrate, $\mathrm{C}_{10} \mathrm{H}_{11} \mathrm{~N}_{4} \mathrm{O}_{2} \mathrm{~S}^{+} \cdot \mathrm{NO}_{3}{ }^{-}$, (VI)); an ethanesulfonate $\{4-[$ (pyrimidin-2-yl)sulfamoyl] anilinium ethanesulfonate, $\mathrm{C}_{10} \mathrm{H}_{11} \mathrm{~N}_{4} \mathrm{O}_{2} \mathrm{~S}^{+} \cdot \mathrm{C}_{2} \mathrm{H}_{5} \mathrm{SO}_{3}{ }^{-}$, (VII) \}; and a dihydrate of the 4-hydroxybenzenesulfonate $\{4-[$ (pyrimidin-2-yl)sulfamoyl]anilinium 4-hydroxybenzenesulfonate dihydrate, $\mathrm{C}_{10} \mathrm{H}_{11}$ $\mathrm{N}_{4} \mathrm{O}_{2} \mathrm{~S}^{+} \cdot \mathrm{HOC}_{6} \mathrm{H}_{4} \mathrm{SO}_{3}{ }^{-} \cdot 2 \mathrm{H}_{2} \mathrm{O}$, (VIII) $\}$. All these structures feature alternate layers of cations and of anions where any solvent is associated with the anion layers. The two sulfonate salts are protonated at the aniline $\mathrm{N}$ atom and the amide $\mathrm{N}$ atom of sulfadiazine, a tautomeric form of the sulfadiazine cation that has not been crystallographically described before. All the other salt forms are instead protonated at the aniline group and on one $\mathrm{N}$ atom of the pyrimidine ring. Whilst all eight species are based upon hydrogen-bonded centrosymetric dimers with graph set $R_{2}^{2}(8)$, the two sulfonate structures also differ in that these dimers do not link into one-dimensional chains of cations through $\mathrm{NH}_{3}$-to- $\mathrm{SO}_{2}$ hydrogen-bonding interactions, whilst the other six species do. The chloride methanol hemisolvate and the tetraiodide are isostructural and a packing analysis of the cation positions shows that the chloride monohydrate structure is also closely related to these.

Keywords: crystal structure; sulfa drugs; sulfadiazine; salt forms; tautomerism; pharmaceutical compounds; tetraiodide; active pharmaceutical ingredients (APIs).

\section{Introduction}

Sulfadiazine [systematic name: 4-amino- $N$-(pyrimidin-2-yl)benzenesulfonamide] is a sulfonamide antibiotic which is often used in the form of an $\mathrm{Ag}^{\mathrm{I}}$ complex within a topical cream (Fisher et al., 2003). Although crystal structures of both sulfadiazine and its $\mathrm{Ag}^{\mathrm{I}}$ complex have been known for some time (Kokila et al., 1995; Cook \& Turner, 1975), and despite the general interest in modifying physicochemical properties of Active Pharmaceutical Ingredients (APIs) by salt formation (Stahl \& Wermuth, 2008), it is only recently that Englert and co-workers described the first structures of salt forms containing protonated sulfadiazine cations (Pan et al., 2013). That study on two solvated forms of sulfadiazine hydrochloride used charge-density measurements to probe the

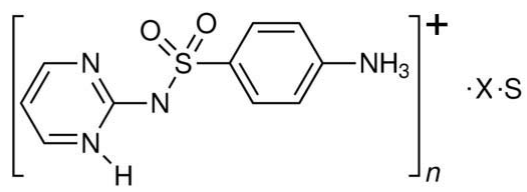

(I) $\mathrm{X}=\mathrm{Cl}^{-}, n=1, \mathrm{~S}=\mathrm{H}_{2} \mathrm{O}$

(II) $\mathrm{X}=\mathrm{Cl}^{-}, n=1, \mathrm{~S}=0.5 \mathrm{MeOH}$

(III) $\mathrm{X}=\mathrm{Br}^{-}, n=1, \mathrm{~S}=\mathrm{H}_{2} \mathrm{O}$

(IV) $\mathrm{X}=\mathrm{I}_{4}^{2-}, n=2$

(V) $\mathrm{X}=\mathrm{BF}_{4}^{-}, n=1, \mathrm{~S}=\mathrm{H}_{2} \mathrm{O}$

(VI) $\mathrm{X}=\mathrm{NO}_{3}^{-}, n=1$

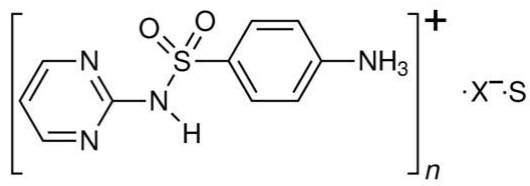

(VII) $\mathrm{X}=\mathrm{EtSO}_{3}, n=1$

(VIII) $\mathrm{X}=\mathrm{HOC}_{6} \mathrm{H}_{4} \mathrm{SO}_{3}, n=1, \mathrm{~S}=2 \mathrm{H}_{2} \mathrm{O}$

unusual protonation behaviour of sulfadiazine - which was shown to form cations by protonating two $\mathrm{N}$ atoms of the aniline group and the pyrimidine ring whilst leaving the amide $\mathrm{N}$ atom unprotonated and formally carrying a negative charge. This is contrary to what may be expected based on $\mathrm{p} K_{a}$ values and to what has been observed for cations of the related sulfonamide drug sulfamethazine (Smith \& Wermuth, 2013a,b; Lu et al., 2008), where the weakly basic aniline group remains neutral as an $\mathrm{NH}_{2}$ species. In order to further examine this feature, we extend the number of sulfadiazine salt structues known by presenting eight new structures, viz. compounds (I)(VIII) (see Scheme). 
Table 1

Experimental details.

For all refinements, $\mathrm{H}$ atoms were treated by a mixture of independent and constrained refinement.

\begin{tabular}{|c|c|c|c|c|}
\hline & (I) & (II) & (III) & (IV) \\
\hline \multicolumn{5}{|l|}{ Crystal data } \\
\hline Chemical formula & $\mathrm{C}_{10} \mathrm{H}_{11} \mathrm{~N}_{4} \mathrm{O}_{2} \mathrm{~S}^{+} \cdot \mathrm{Cl}^{-} \cdot \mathrm{H}_{2} \mathrm{O}$ & $\mathrm{C}_{10} \mathrm{H}_{11} \mathrm{~N}_{4} \mathrm{O}_{2} \mathrm{~S}^{+} \cdot \mathrm{Cl}^{-} \cdot 0.5 \mathrm{CH}_{4} \mathrm{O}$ & $\mathrm{C}_{10} \mathrm{H}_{11} \mathrm{~N}_{4} \mathrm{O}_{2} \mathrm{~S}^{+} \cdot \mathrm{Br}^{-} \cdot \mathrm{H}_{2} \mathrm{O}$ & $2 \mathrm{C}_{10} \mathrm{H}_{11} \mathrm{~N}_{4} \mathrm{O}_{2} \mathrm{~S}^{+} \cdot \mathrm{I}_{4}{ }^{2-}$ \\
\hline$M_{\mathrm{r}}$ & 304.75 & 302.76 & 349.21 & 1010.18 \\
\hline Crystal system, space group & Triclinic, $P \overline{1}$ & Triclinic, $P \overline{1}$ & Monoclinic, $P 2_{1} / n$ & Triclinic, $P \overline{1}$ \\
\hline Temperature $(\mathrm{K})$ & 100 & 100 & 123 & 123 \\
\hline$a, b, c(\AA)$ & $\begin{array}{l}5.4118(4), 11.5632(8) \\
\quad 11.7430(8)\end{array}$ & $\begin{array}{l}5.6468(2), 11.2749(4) \\
\quad 11.8363(8)\end{array}$ & $\begin{array}{l}11.9420(3), 5.6295(2) \\
21.0659(6)\end{array}$ & $\begin{array}{l}5.7792(4), 12.0002(7) \\
\quad 12.5214(9)\end{array}$ \\
\hline$\alpha, \beta, \gamma\left({ }^{\circ}\right)$ & $\begin{array}{l}109.462(7), 94.399(6) \\
102.509(7)\end{array}$ & $\begin{array}{l}64.254(5), 79.361(6) \\
\quad 77.687(6)\end{array}$ & $90,98.380(3), 90$ & $\begin{array}{l}61.964(7), 87.745(5) \\
\quad 76.426(5)\end{array}$ \\
\hline$V\left(\AA^{3}\right)$ & $667.51(8)$ & $659.53(6)$ & $1401.09(7)$ & $742.51(9)$ \\
\hline$Z$ & 2 & 2 & 4 & 1 \\
\hline Radiation type & Mo $K \alpha$ & Mo $K \alpha$ & Mo $K \alpha$ & Mo $K \alpha$ \\
\hline$\mu\left(\mathrm{mm}^{-1}\right)$ & 0.45 & 0.45 & 3.09 & 4.38 \\
\hline Crystal size (mm) & $0.06 \times 0.02 \times 0.01$ & $0.09 \times 0.06 \times 0.05$ & $0.30 \times 0.22 \times 0.04$ & $0.25 \times 0.15 \times 0.02$ \\
\hline \multicolumn{5}{|l|}{ Data collection } \\
\hline Diffractometer & $\begin{array}{l}\text { Rigaku Saturn } 724+(2 \times 2 \\
\text { bin mode) diffractometer }\end{array}$ & $\begin{array}{l}\text { Rigaku Saturn } 724+(2 \times 2 \\
\text { bin mode) diffractometer }\end{array}$ & $\begin{array}{l}\text { Oxford Diffraction } \\
\text { Xcalibur E diffractometer }\end{array}$ & $\begin{array}{l}\text { Oxford Diffraction } \\
\text { Xcalibur E diffractometer }\end{array}$ \\
\hline Absorption correction & $\begin{array}{c}\text { Multi-scan (CrystalClear-SM } \\
\text { Expert; Rigaku, 2013) }\end{array}$ & $\begin{array}{c}\text { Multi-scan (CrystalClear-SM } \\
\text { Expert; Rigaku, 2013) }\end{array}$ & $\begin{array}{l}\text { Multi-scan (CrysAlis PRO; } \\
\text { Oxford Diffraction, 2010) }\end{array}$ & $\begin{array}{l}\text { Analytical [CrysAlis PRO } \\
\text { (Oxford Diffraction, 2010), } \\
\text { based on expressions } \\
\text { derived by Clark \& Reid } \\
(1995)]\end{array}$ \\
\hline$T_{\min }, T_{\max }$ & $0.897,1.000$ & $0.819,1.000$ & $0.616,1.000$ & $0.523,0.905$ \\
\hline $\begin{array}{l}\text { No. of measured, independent and } \\
\text { observed }[I>2 \sigma(I)] \text { reflections }\end{array}$ & $8329,3000,2752$ & $11731,3028,2804$ & $8186,3353,2868$ & $7055,3595,2996$ \\
\hline$R_{\text {int }}$ & 0.025 & 0.029 & 0.024 & 0.033 \\
\hline$(\sin \theta / \lambda)_{\max }\left(\AA^{-1}\right)$ & 0.649 & 0.649 & 0.661 & 0.691 \\
\hline \multicolumn{5}{|l|}{ Refinement } \\
\hline$R\left[F^{2}>2 \sigma\left(F^{2}\right)\right], w R\left(F^{2}\right), S$ & $0.029,0.079,1.05$ & $0.031,0.082,1.04$ & $0.029,0.072,1.06$ & $0.032,0.062,1.04$ \\
\hline No. of reflections & 3000 & 3028 & 3353 & 3595 \\
\hline No. of parameters & 197 & 201 & 203 & 184 \\
\hline No. of restraints & 5 & 1 & 5 & 0 \\
\hline \multirow[t]{2}{*}{$\Delta \rho_{\max }, \Delta \rho_{\min }\left(\mathrm{e} \AA^{-3}\right)$} & $0.51,-0.38$ & $0.40,-0.43$ & $0.46,-0.52$ & $0.66,-0.93$ \\
\hline & $(\mathrm{V})$ & $(\mathrm{VI})$ & (VII) & (VIII) \\
\hline \multicolumn{5}{|l|}{ Crystal data } \\
\hline Chemical formula & $\mathrm{C}_{10} \mathrm{H}_{11} \mathrm{~N}_{4} \mathrm{O}_{2} \mathrm{~S}^{+} \cdot \mathrm{BF}_{4}^{-} \cdot \mathrm{H}_{2} \mathrm{O}$ & $\mathrm{C}_{10} \mathrm{H}_{11} \mathrm{~N}_{4} \mathrm{O}_{2} \mathrm{~S}^{+} \cdot \mathrm{NO}_{3}{ }^{-}$ & $\mathrm{C}_{10} \mathrm{H}_{11} \mathrm{~N}_{4} \mathrm{O}_{2} \mathrm{~S}^{+} \cdot \mathrm{C}_{2} \mathrm{H}_{5} \mathrm{O}_{3} \mathrm{~S}^{-}$ & $\mathrm{C}_{10} \mathrm{H}_{11} \mathrm{~N}_{4} \mathrm{O}_{2} \mathrm{~S}^{+} \cdot \mathrm{C}_{6} \mathrm{H}_{5} \mathrm{O}_{4} \mathrm{~S}^{-} \cdot 2 \mathrm{H}_{2} \mathrm{O}$ \\
\hline$M_{\mathrm{r}}$ & 356.11 & 313.30 & 360.41 & 460.48 \\
\hline Crystal system, space group & Monoclinic, $P 2_{1} / n$ & Triclinic, $P \overline{1}$ & Monoclinic, $P 2_{1} / c$ & Monoclinic, $P 2_{1} / n$ \\
\hline Temperature (K) & 123 & 123 & 123 & 123 \\
\hline$a, b, c(\AA)$ & $\begin{array}{l}5.8539(1), 11.3629(3) \\
\quad 21.2080(6)\end{array}$ & $\begin{array}{l}5.4705(4), 10.1235(7) \\
\quad 11.9956(8)\end{array}$ & $\begin{array}{l}5.5269(4), 34.979(3) \\
\quad 8.4395(6)\end{array}$ & $\begin{array}{l}18.5872(5), 5.9733(2) \\
\quad 18.6850(5)\end{array}$ \\
\hline$\alpha, \beta, \gamma\left({ }^{\circ}\right)$ & $90,90.982(2), 90$ & $\begin{array}{l}86.708(6), 85.489(6) \\
\quad 74.394(7)\end{array}$ & $90,93.102(7), 90$ & $90,104.858(3), 90$ \\
\hline$V\left(\AA^{3}\right)$ & $1410.49(6)$ & $637.40(8)$ & $1629.2(2)$ & $2005.17(10)$ \\
\hline$Z$ & 4 & 2 & 4 & 4 \\
\hline Radiation type & Mo $K \alpha$ & Mo $K \alpha$ & Mo $K \alpha$ & Mo $K \alpha$ \\
\hline$\mu\left(\mathrm{mm}^{-1}\right)$ & 0.30 & 0.29 & 0.36 & 0.32 \\
\hline Crystal size $(\mathrm{mm})$ & $0.20 \times 0.12 \times 0.05$ & $0.26 \times 0.15 \times 0.06$ & $0.4 \times 0.03 \times 0.02$ & $0.20 \times 0.12 \times 0.05$ \\
\hline \multicolumn{5}{|l|}{ Data collection } \\
\hline Diffractometer & $\begin{array}{l}\text { Oxford Diffraction } \\
\text { Xcalibur E diffractometer }\end{array}$ & $\begin{array}{l}\text { Oxford Diffraction } \\
\text { Xcalibur E diffractometer }\end{array}$ & $\begin{array}{l}\text { Oxford Diffraction } \\
\text { Xcalibur E diffractometer }\end{array}$ & $\begin{array}{l}\text { Oxford Diffraction } \\
\text { Xcalibur E diffractometer }\end{array}$ \\
\hline Absorption correction & $\begin{array}{l}\text { Multi-scan (CrysAlis PRO; } \\
\text { Oxford Diffraction, 2010) }\end{array}$ & $\begin{array}{l}\text { Multi-scan (CrysAlis PRO; } \\
\text { Oxford Diffraction, 2010) }\end{array}$ & $\begin{array}{l}\text { Multi-scan (CrysAlis PRO; } \\
\text { Oxford Diffraction, 2010) }\end{array}$ & $\begin{array}{l}\text { Multi-scan (CrysAlis PRO; } \\
\text { Oxford Diffraction, 2010) }\end{array}$ \\
\hline$T_{\min }, T_{\max }$ & $0.874,1.000$ & $0.897,1.000$ & $0.908,1.000$ & $0.968,1.000$ \\
\hline $\begin{array}{l}\text { No. of measured, independent and } \\
\text { observed }[I>2 \sigma(I)] \text { reflections }\end{array}$ & $8710,3549,2955$ & $7390,2977,2429$ & $19991,3515,2212$ & $9653,4695,3638$ \\
\hline$R_{\text {int }}$ & 0.024 & 0.026 & 0.104 & 0.029 \\
\hline$(\sin \theta / \lambda)_{\max }\left(\AA^{-1}\right)$ & 0.682 & 0.661 & 0.639 & 0.661 \\
\hline \multicolumn{5}{|l|}{ Refinement } \\
\hline$R\left[F^{2}>2 \sigma\left(F^{2}\right)\right], w R\left(F^{2}\right), S$ & $0.037,0.089,1.03$ & $0.045,0.110,1.04$ & $0.055,0.112,1.01$ & $0.048,0.113,1.03$ \\
\hline No. of reflections & 3549 & 2977 & 3515 & 4695 \\
\hline No. of parameters & 232 & 206 & 222 & 307 \\
\hline No. of restraints & 3 & 0 & 3 & 6 \\
\hline$\Delta \rho_{\max }, \Delta \rho_{\min }\left(\mathrm{e} \AA^{-3}\right)$ & $0.35,-0.46$ & $0.78,-0.38$ & $0.35,-0.41$ & $0.80,-0.44$ \\
\hline
\end{tabular}

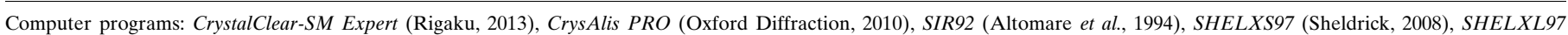
(Sheldrick, 2008), ORTEP-3 for Windows (Farrugia, 2012), Mercury (Macrae et al., 2008) and X-SEED (Barbour, 2001). 


\section{Experimental}

\subsection{Synthesis and crystallization}

Following the method of Buist et al. (2013), chloride methanol hemisolvate (II) was prepared by adding acetyl chloride $(1 \mathrm{ml})$ to a methanol solution $(4 \mathrm{ml})$ of sulfadiazine $(0.226 \mathrm{~g})$. Crystals were grown directly from the reaction mixture by slow evaporation of the solvent. All other compounds were obtained by adding the appropriate acid to aqueous solutions of sulfadiazine in water, filtering to obtain clear solutions, and then allowing slow evaporation of the solvent to promote crystal growth. Solid-state IR spectra were measured with an $\mathrm{A}_{2}$ Technologies ATR instrument and are included in the Supporting information.

\subsection{Refinement}

Crystal data, data collection and structure refinement details are summarized in Table 1. For all eight title structures, $\mathrm{H}$ atoms bound to $\mathrm{C}$ atoms were placed in the expected geometric positions and treated in riding modes, with $\mathrm{C}-\mathrm{H}=$ $0.95 \AA$ and $U_{\text {iso }}(\mathrm{H})=1.2 U_{\text {eq }}(\mathrm{C})$, except for methyl $\mathrm{H}$ atoms, for which $\mathrm{C}-\mathrm{H}=0.98 \AA$ and $U_{\text {iso }}(\mathrm{H})=1.5 U_{\text {eq }}(\mathrm{C})$. With the exceptions noted below, $\mathrm{H}$ atoms bound to $\mathrm{O}$ or $\mathrm{N}$ atoms were placed as found and refined isotropically. For all hydrates, the $\mathrm{O}-\mathrm{H}$ and $\mathrm{H} \cdots \mathrm{H}$ distances of the water molecules were restrained to 0.88 (1) and 1.33 (2) $\AA$, respectively. Similarly, the $\mathrm{O}-\mathrm{H}$ distance of the methanol solvent molecule in (II) was restrained to 0.88 (1) $\AA$ and the azanium $\mathrm{N}-\mathrm{H}$ distances in (VII) were restrained to 0.91 (1) ^. For (I), (II) and (III), the solvent $\mathrm{H}$ atoms were also given displacement parameters such that $U_{\text {iso }}(\mathrm{H})=1.2 U_{\text {eq }}(\mathrm{O})$. For $(\mathrm{I})$, atoms $\mathrm{H} 2 W$ and $\mathrm{H} 3 W$ have site-occupancy factors of 0.5 . For tetraiodide (IV), it was found neccessary to set the $U_{\text {iso }}(\mathrm{H})$ values at $1.2 U_{\text {eq }}(\mathrm{N})$ for the $\mathrm{H}$ atoms bound to $\mathrm{N}$ atoms. For (III), the water molecule was found to be disordered over several sites that formed a channel. In the final structure, after numerous trial calculations, this was modelled as four O-atom sites. These were constrained to have equal displacement parameters and their individual site-occupancy parameters were allowed to refine independently but were restrained to sum to unity. This gave final values of 0.453 (10), 0.248 (10), 0.161 (6) and 0.139 (4) for $\mathrm{O} 1 W, \mathrm{O} 2 W, \mathrm{O} 3 W$ and $\mathrm{O} 4 W$, respectively. For these disordered sites, $\mathrm{H}$ atoms could only be placed on the major component, i.e. $\mathrm{O} 1 W$, with $U_{\text {iso }}(\mathrm{H})=1.5 U_{\text {eq }}(\mathrm{O})$. For the amide $\mathrm{H}$ atom in (III), $U_{\text {iso }}(\mathrm{H})$ values were set at $1.2 U_{\text {eq }}(\mathrm{N})$ and for the anilinium groups in (III) and (VII) they were set at $1.5 U_{\mathrm{eq}}(\mathrm{N})$.

\section{Results and discussion}

Two chloride-containing salt phases were prepared, i.e. a chloride monohydrate, (I) (Fig. 1), obtained from aqueous solution and a methanol hemisolvate, (II) (Fig. 2), obtained by reacting sulfadiazine with acetyl chloride in methanol [see Buist et al. (2014) for another example of the use of these reagents for obtaining differently solvated forms of APIs]. Methanol hemisolvate (II) is isostructural with the ethanol

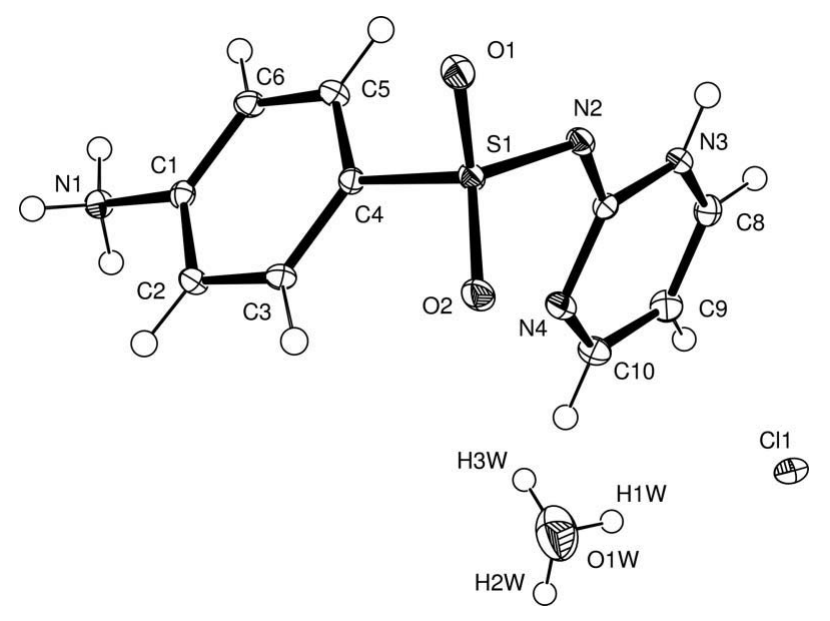

Figure 1

The molecular structure of (I), with non-H atoms shown as $50 \%$ probability displacement ellipsoids. The $\mathrm{H} 2 W$ and $\mathrm{H} 3 W$ sites are disordered and both have site-occupancy factors of 0.5 .

and ethylene glycol solvates described previously by Pan et al. (2013). All three isostructural forms feature a solvent molecule at a crystallographic inversion centre. For the methanol hemisolvate, (II), and ethanol species this results in a disordered solvent site with half-occupancy atoms. Chloride monohydrate (I) also has a very similar structure, as can be shown by constructing 20-molecule overlays in Mercury (Macrae et al., 2008; Allen, 2002). These confirm that all four chloride forms have 20 out of 20 cation positions in common (when software option 'ignore smallest molecular components' is selected and 30\% geometric tolerances are allowed). For (I), the smaller solvent size means that two water molecules can be accommodated at the solvent site with no Oatom disorder. The $\mathrm{H}$ atoms of the water molecule are, however, disordered, with $\mathrm{H} 2 W$ and $\mathrm{H} 3 W$ being alternative sites both with site-occupancy factors of 0.5 . These disordered $\mathrm{H}$ atoms take part in water-to-water hydrogen-bond contacts which give polymeric chains of connected water molecules that extend parallel to the crystallographic $a$ direction. The remaining well-ordered $\mathrm{H}$ atom, $\mathrm{H} 1 \mathrm{~W}$, forms a hydrogen bond to the chloride ion. The three organic solvates cannot form

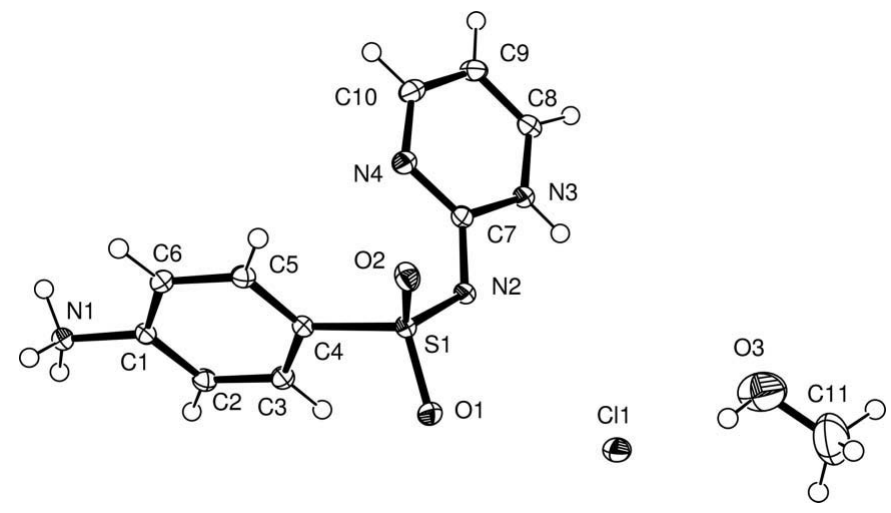

Figure 2

The molecular structure of (II), with non-H atoms shown as $50 \%$ probability displacement ellipsoids. All atoms of the methanol solvent molecule have site-occupancy factors of 0.5 . 
Table 2

Hydrogen-bond geometry $\left(\AA,^{\circ}\right)$ for (I).

\begin{tabular}{lllll}
\hline$D-\mathrm{H} \cdots A$ & $D-\mathrm{H}$ & $\mathrm{H} \cdots A$ & $D \cdots A$ & $D-\mathrm{H} \cdots A$ \\
\hline $\mathrm{N} 1-\mathrm{H} 1 \mathrm{~N} \cdots \mathrm{Cl} 1^{\mathrm{i}}$ & $0.91(2)$ & $2.27(2)$ & $3.1484(13)$ & $161.8(19)$ \\
$\mathrm{N} 1-\mathrm{H} 2 \mathrm{~N} \cdots \mathrm{Cl} 1^{\text {ii }}$ & $0.91(2)$ & $2.27(2)$ & $3.1795(13)$ & $176.0(18)$ \\
$\mathrm{N} 1-\mathrm{H} 3 \mathrm{~N} \cdots \mathrm{O} 1^{\text {ii }}$ & $0.91(2)$ & $1.99(2)$ & $2.8243(16)$ & $150.2(19)$ \\
$\mathrm{N} 3-\mathrm{H} 4 \mathrm{~N} \cdots 2^{\text {iv }}$ & $0.90(2)$ & $1.98(2)$ & $2.8763(17)$ & $175.9(19)$ \\
$\mathrm{N} 3-\mathrm{H} 4 \mathrm{~N} \cdots \mathrm{O} 1^{\text {iv }}$ & $0.90(2)$ & $2.54(2)$ & $3.0962(15)$ & $121.1(16)$ \\
$\mathrm{O} 1 W-\mathrm{H} 1 W \cdots \mathrm{Cl} 1$ & $0.88(1)$ & $2.62(1)$ & $3.4607(17)$ & $160(3)$ \\
$\mathrm{O} 1 W-\mathrm{H} 2 W \cdots \mathrm{O} 1 W^{\mathrm{v}}$ & $0.88(1)$ & $2.18(2)$ & $2.995(4)$ & $153(4)$ \\
$\mathrm{O} 1 W-\mathrm{H} 3 W \cdots \mathrm{O} 1 W^{\mathrm{i}}$ & $0.88(1)$ & $2.21(2)$ & $3.030(4)$ & $155(5)$ \\
\hline
\end{tabular}

Symmetry codes: (i) $-x+2,-y+2,-z+1$; (ii) $-x+1,-y+2,-z+1$; (iii) $-x,-y+1,-z$; (iv) $-x,-y+1,-z+1 ;$ (v) $-x+3,-y+2,-z+1$.

Table 3

Hydrogen-bond geometry $\left(\AA{ }^{\circ}\right)$ for (II).

\begin{tabular}{lllll}
\hline$D-\mathrm{H} \cdots A$ & $D-\mathrm{H}$ & $\mathrm{H} \cdots A$ & $D \cdots A$ & $D-\mathrm{H} \cdots A$ \\
\hline $\mathrm{N} 1-\mathrm{H} 1 \mathrm{~N} \cdots \mathrm{Cl} 1^{\mathrm{i}}$ & $0.91(2)$ & $2.30(2)$ & $3.2088(14)$ & $174.1(18)$ \\
$\mathrm{N} 1-\mathrm{H} 2 \mathrm{~N} \cdots \mathrm{Cl} 1^{\text {ii }}$ & $0.86(2)$ & $2.70(2)$ & $3.2151(13)$ & $119.8(18)$ \\
$\mathrm{N} 1-\mathrm{H} 2 \mathrm{~N} \cdots \mathrm{O} 1^{\mathrm{ii}}$ & $0.86(2)$ & $2.13(2)$ & $2.8701(18)$ & $144(2)$ \\
$\mathrm{N} 1-\mathrm{H} 3 \mathrm{~N} \cdots \mathrm{Cl} 1^{\text {ii }}$ & $0.92(3)$ & $2.25(3)$ & $3.1417(14)$ & $163(2)$ \\
$\mathrm{N} 3-\mathrm{H} 4 \mathrm{~N} \cdots \mathrm{N} 2^{\text {iv }}$ & $0.89(2)$ & $2.01(2)$ & $2.8957(18)$ & $175.4(19)$ \\
$\mathrm{N} 3-\mathrm{H} 4 \mathrm{~N} \cdots \mathrm{O} 1^{\text {iv }}$ & $0.89(2)$ & $2.59(2)$ & $3.1218(16)$ & $119.6(16)$ \\
$\mathrm{O} 3-\mathrm{H} 1 \mathrm{H} \cdots \mathrm{Cl} 1$ & $0.89(1)$ & $2.31(2)$ & $3.170(3)$ & $163(6)$ \\
\hline
\end{tabular}

Symmetry codes: (i) $x, y+1, z$; (ii) $-x+1,-y+2,-z$; (iii) $x-1, y+1, z$; (iv) $-x+1,-y+1,-z+1$.

Table 4

Hydrogen-bond geometry $\left(\AA{ }^{\circ}\right)$ for (III).

\begin{tabular}{lllll}
\hline$D-\mathrm{H} \cdots A$ & $D-\mathrm{H}$ & $\mathrm{H} \cdots A$ & $D \cdots A$ & $D-\mathrm{H} \cdots A$ \\
\hline $\mathrm{N} 1-\mathrm{H} 1 \mathrm{~N} \cdots \mathrm{Br} 1$ & $0.91(3)$ & $2.44(3)$ & $3.308(2)$ & $159(2)$ \\
$\mathrm{N} 1-\mathrm{H} 2 \mathrm{~N} \cdots 2^{\mathrm{i}}$ & $0.88(3)$ & $2.01(3)$ & $2.810(3)$ & $150(3)$ \\
$\mathrm{N} 1-\mathrm{H} 2 \mathrm{~N} \cdots \mathrm{Br}^{\mathrm{ii}}{ }^{\mathrm{ii}}$ & $0.88(3)$ & $2.95(3)$ & $3.4022(18)$ & $114(2)$ \\
$\mathrm{N} 1-\mathrm{H} 3 \mathrm{~N} \cdots \mathrm{Br}^{\text {iii }}$ & $0.85(3)$ & $2.46(3)$ & $3.317(2)$ & $178(2)$ \\
$\mathrm{N} 3-\mathrm{H} 4 \mathrm{~N} \cdots 2^{\mathrm{i}}$ & $0.87(1)$ & $2.02(1)$ & $2.887(2)$ & $179(2)$ \\
$\mathrm{N} 3-\mathrm{H} 4 \mathrm{~N} \cdots 2^{\text {iv }}$ & $0.87(1)$ & $2.57(2)$ & $3.080(2)$ & $119(2)$ \\
\hline
\end{tabular}

Symmetry codes: (i) $-x+1,-y+2,-z+2$; (ii) $-x+\frac{1}{2}, y+\frac{1}{2},-z+\frac{3}{2}$; (iii) $x, y+1, z$; (iv) $-x+2,-y+2,-z+2$.

Table 5

Hydrogen-bond geometry $\left(\AA,^{\circ}\right)$ for (IV).

\begin{tabular}{lllll}
\hline$D-\mathrm{H} \cdots A$ & $D-\mathrm{H}$ & $\mathrm{H} \cdots A$ & $D \cdots A$ & $D-\mathrm{H} \cdots A$ \\
\hline $\mathrm{N} 1-\mathrm{H} 1 \mathrm{~N} \cdots \mathrm{I} 1^{\mathrm{i}}$ & $0.85(4)$ & $2.64(4)$ & $3.482(4)$ & $172(4)$ \\
$\mathrm{N} 1-\mathrm{H} 2 \mathrm{~N} \cdots \mathrm{O} 2^{\text {ii }}$ & $0.84(4)$ & $2.00(4)$ & $2.798(4)$ & $158(4)$ \\
$\mathrm{N} 1-\mathrm{H} 3 \mathrm{~N} \cdots \mathrm{I} 1$ & $0.96(4)$ & $2.59(4)$ & $3.524(3)$ & $165(3)$ \\
$\mathrm{N} 4-\mathrm{H} 4 \mathrm{~N} \cdots \mathrm{N} 2^{\text {iii }}$ & $0.91(4)$ & $2.00(4)$ & $2.906(5)$ & $174(4)$ \\
\hline
\end{tabular}

Symmetry codes: (i) $x+1, y, z$; (ii) $-x,-y+2,-z+1$; (iii) $-x,-y+2,-z+2$.

\section{Table 6}

Hydrogen-bond geometry $\left(\AA{ }^{\circ}\right)$ for $(\mathrm{V})$.

\begin{tabular}{lllll}
\hline$D-\mathrm{H} \cdots A$ & $D-\mathrm{H}$ & $\mathrm{H} \cdots A$ & $D \cdots A$ & $D-\mathrm{H} \cdots A$ \\
\hline $\mathrm{N} 1-\mathrm{H} 1 \mathrm{~N} \cdots \mathrm{F} 3^{\mathrm{i}}$ & $0.93(2)$ & $1.92(2)$ & $2.7912(17)$ & $156.0(19)$ \\
$\mathrm{N} 1-\mathrm{H} 2 \mathrm{~N} \cdots \mathrm{O} 2^{\text {ii }}$ & $0.88(2)$ & $2.05(2)$ & $2.8196(19)$ & $144.7(18)$ \\
$\mathrm{N} 1-\mathrm{H} 3 \mathrm{~N} \cdots \mathrm{O} 1 W$ & $0.92(2)$ & $1.87(3)$ & $2.7762(19)$ & $166(2)$ \\
$\mathrm{N} 3-\mathrm{H} 4 \mathrm{~N} \cdots \mathrm{N} 2^{\text {iii }}$ & $0.89(2)$ & $2.02(2)$ & $2.9092(17)$ & $176.8(19)$ \\
N3-H4N $\cdots \mathrm{O} 2^{\text {iii }}$ & $0.89(2)$ & $2.58(2)$ & $3.1307(17)$ & $121.2(15)$ \\
$\mathrm{O} 1 W-\mathrm{H} 1 W \cdots \mathrm{F}{ }^{\text {iv }}$ & $0.87(1)$ & $2.03(1)$ & $2.8790(15)$ & $165(2)$ \\
O1 $W-\mathrm{H} 2 W \cdots \mathrm{F} 1$ & $0.88(1)$ & $1.94(1)$ & $2.7863(17)$ & $163(2)$ \\
\hline
\end{tabular}

Symmetry codes: (i) $-x+\frac{1}{2}, y+\frac{1}{2},-z+\frac{3}{2}$; (ii) $-x,-y+1,-z+2$; (iii) $-x,-y,-z+2$; (iv) $x-1, y, z$.
Table 7

Hydrogen-bond geometry $\left(\AA,^{\circ}\right)$ for (VI).

\begin{tabular}{lllll}
\hline$D-\mathrm{H} \cdots A$ & $D-\mathrm{H}$ & $\mathrm{H} \cdots A$ & $D \cdots A$ & $D-\mathrm{H} \cdots A$ \\
\hline $\mathrm{N} 1-\mathrm{H} 1 \mathrm{~N} \cdots \mathrm{O}^{\mathrm{i}}$ & $0.96(3)$ & $1.85(3)$ & $2.803(3)$ & $171(3)$ \\
$\mathrm{N} 1-\mathrm{H} 1 \mathrm{~N} \cdots \mathrm{O} 4^{\mathrm{i}}$ & $0.96(3)$ & $2.42(3)$ & $2.968(3)$ & $116(2)$ \\
$\mathrm{N} 1-\mathrm{H} 2 \mathrm{~N} \cdots \mathrm{O} 3$ & $0.85(3)$ & $2.31(3)$ & $2.912(3)$ & $128(3)$ \\
$\mathrm{N} 1-\mathrm{H} 2 \mathrm{~N} \cdots \mathrm{O} 5$ & $0.85(3)$ & $2.32(3)$ & $3.021(3)$ & $140(3)$ \\
$\mathrm{N} 1-\mathrm{H} 3 \mathrm{~N} \cdots \mathrm{O} 1^{\mathrm{ii}}$ & $0.95(3)$ & $1.85(3)$ & $2.784(2)$ & $166(3)$ \\
$\mathrm{N} 3-\mathrm{H} 4 \mathrm{~N} \cdots \mathrm{N} 2^{\mathrm{iii}}$ & $0.85(3)$ & $2.06(3)$ & $2.911(3)$ & $176(2)$ \\
$\mathrm{N} 3-\mathrm{H} 4 \mathrm{~N} \cdots \mathrm{O} 1^{\mathrm{iii}}$ & $0.85(3)$ & $2.55(3)$ & $3.085(2)$ & $122(2)$ \\
\hline
\end{tabular}

Symmetry codes: (i) $x+1, y, z$; (ii) $-x+1,-y,-z+1$; (iii) $-x+1,-y,-z+2$.

Table 8

Hydrogen-bond geometry $\left(\AA{ }^{\circ}\right)$ for (VII).

\begin{tabular}{lllll}
\hline$D-\mathrm{H} \cdots A$ & $D-\mathrm{H}$ & $\mathrm{H} \cdots A$ & $D \cdots A$ & $D-\mathrm{H} \cdots A$ \\
\hline $\mathrm{N} 1-\mathrm{H} 1 \mathrm{~N} \cdots \mathrm{O}^{\mathrm{i}}$ & $0.91(1)$ & $2.05(1)$ & $2.929(4)$ & $164(3)$ \\
$\mathrm{N} 1-\mathrm{H} 2 \mathrm{~N} \cdots 4^{\mathrm{ii}}$ & $0.90(1)$ & $2.17(2)$ & $2.980(4)$ & $149(3)$ \\
$\mathrm{N} 1-\mathrm{H} 2 \mathrm{~N} \cdots 3^{\mathrm{iii}}$ & $0.90(1)$ & $2.26(3)$ & $2.705(3)$ & $110(2)$ \\
$\mathrm{N} 1-\mathrm{H} 3 \mathrm{~N} \cdots \mathrm{O} 5$ & $0.91(1)$ & $1.89(1)$ & $2.801(3)$ & $176(3)$ \\
$\mathrm{N} 2-\mathrm{H} 4 \mathrm{~N} \cdots \mathrm{N} 4^{\mathrm{iv}}$ & $0.89(3)$ & $1.99(3)$ & $2.882(4)$ & $174(3)$ \\
\hline
\end{tabular}

Symmetry codes: (i) $x,-y+\frac{1}{2}, z+\frac{1}{2}$; (ii) $x+1, y, z$; (iii) $x+1,-y+\frac{1}{2}, z+\frac{1}{2}$; (iv) $-x+1,-y+1,-z+1$.

Table 9

Hydrogen-bond geometry ( $\AA^{\circ}{ }^{\circ}$ ) for (VIII).

\begin{tabular}{lllll}
\hline$D-\mathrm{H} \cdots A$ & $D-\mathrm{H}$ & $\mathrm{H} \cdots A$ & $D \cdots A$ & $D-\mathrm{H} \cdots A$ \\
\hline $\mathrm{N} 1-\mathrm{H} 1 \mathrm{~N} \cdots \mathrm{O} 1 W^{\mathrm{i}}$ & $0.95(3)$ & $1.80(3)$ & $2.733(3)$ & $170(3)$ \\
$\mathrm{N} 1-\mathrm{H} 2 \mathrm{~N} \cdots \mathrm{O} 2 W^{\mathrm{i}}$ & $0.90(3)$ & $2.04(3)$ & $2.873(3)$ & $154(3)$ \\
$\mathrm{N} 1-\mathrm{H} 2 \mathrm{~N} \cdots 5^{\mathrm{ii}}$ & $0.90(3)$ & $2.32(3)$ & $2.793(3)$ & $113(2)$ \\
$\mathrm{N} 1-\mathrm{H} 3 \mathrm{~N} \cdots 6^{\mathrm{iii}}$ & $0.94(3)$ & $1.88(3)$ & $2.780(3)$ & $159(3)$ \\
$\mathrm{N} 2-\mathrm{H} 4 \mathrm{~N} \cdots \mathrm{N} 3^{\mathrm{iv}}$ & $0.87(3)$ & $2.03(3)$ & $2.897(3)$ & $179(3)$ \\
$\mathrm{O} 3-\mathrm{H} 1 \mathrm{H} \cdots \mathrm{O} 2 W$ & $0.96(4)$ & $1.69(4)$ & $2.578(3)$ & $153(4)$ \\
$\mathrm{O} 1 W-\mathrm{H} 1 W \cdots \mathrm{O} 3^{\mathrm{v}}$ & $0.87(1)$ & $1.89(1)$ & $2.760(3)$ & $173(3)$ \\
$\mathrm{O} 1 W-\mathrm{H} 2 W \cdots \mathrm{O} 2^{\text {vi }}$ & $0.87(1)$ & $1.96(1)$ & $2.834(3)$ & $175(3)$ \\
$\mathrm{O} 2 W-\mathrm{H} 3 W \cdots \mathrm{O} 5^{\text {vii }}$ & $0.88(1)$ & $1.85(1)$ & $2.722(3)$ & $172(3)$ \\
$\mathrm{O} 2 W-\mathrm{H} 4 W \cdots \mathrm{O} 4^{\text {ii }}$ & $0.88(1)$ & $1.88(1)$ & $2.737(2)$ & $163(4)$ \\
\hline
\end{tabular}

Symmetry codes: (i) $-x+1,-y+1,-z+1$; (ii) $\quad-x+\frac{1}{2}, y+\frac{1}{2},-z+\frac{1}{2}$; $\quad$ (iii) $-x+\frac{1}{2}, y+\frac{3}{2},-z+\frac{1}{2}$; (iv) $-x+1,-y+2,-z$; (v) $-x+1,-y+2,-z+1$; (vi) $-x+\frac{3}{2}, y+\frac{1}{2},-z+\frac{1}{2}$; (vii) $-x+\frac{1}{2}, y-\frac{1}{2},-z+\frac{1}{2}$.

similar hydrogen-bonded solvent chains and instead form single hydrogen bonds to chloride. See Tables 2-9 for full details of the hydrogen bonding for compounds (I)-(VIII). Together, the water molecules and the chloride ions in (I) lie in sheets parallel to the $a c$ plane. The $\mathrm{V}$-shaped cations pack to give double layers and thus alternating layers of cations and solvent/halide ions exist along the crystallographic $b$ direction (Fig. 3). Layer structures are seen for all the other structures herein.

Reactions with aqueous $\mathrm{HBr}$ and $\mathrm{HI}$ gave the bromide monohydrate, (III) (Fig. 4), and the tetraiodide, (IV) (Fig. 5). Both retain the layered structure described above, but perhaps, anti-intuitively, the hydrated structure with the simple bromide counter-ion is not isostructural with the chloride phases, whereas the anhydrous structure containing $\left[\mathrm{I}_{4}\right]^{2-}$ is isostructural with the solvated chloride structures (Fig. 6), allowing only for a small increase in unit-cell size. This can again be shown by 20 out of 20 sulfadiazine cations having matching positions in a Mercury overlay. Although there are many species of polyiodide known, the $\left[\mathrm{I}_{4}\right]^{2-}$ anion is a rare 


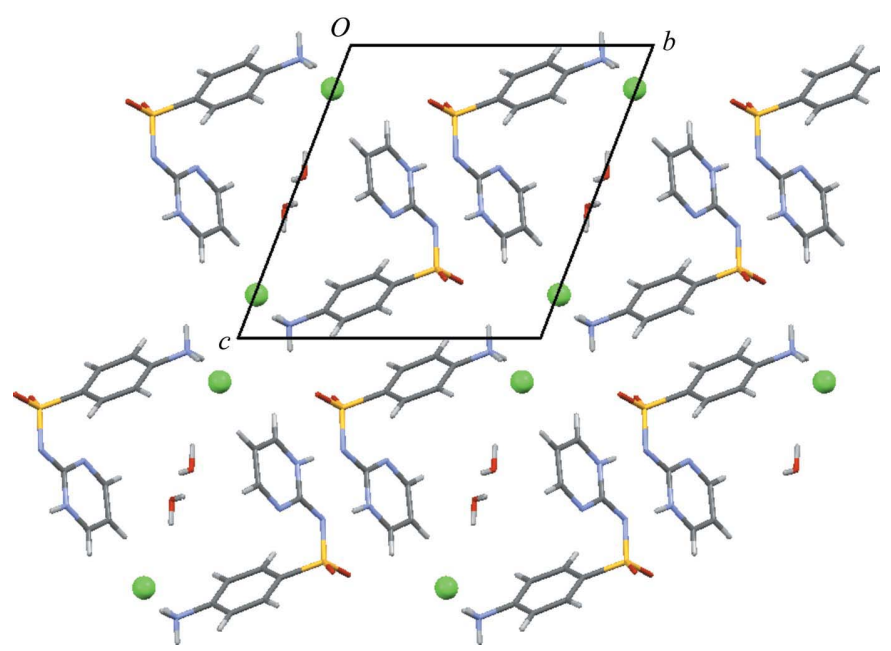

Figure 3

The packing of (I), viewed down the $a$ axis. Note the bilayer of V-shaped cations separated by layers of chloride ions and water molecules. Here and in other figures, $\mathrm{Cl}=$ green, $\mathrm{S}=$ yellow, $\mathrm{O}=$ red and $\mathrm{N}=$ blue.

and thus much sought after variant (Weclawik et al., 2013; Svensson \& Kloo, 2003). Interestingly, a templating approach using a sulfonamide very closely related to sulfadiazine has been shown recently to allow access to tetraiodide species (Pan \& Englert, 2014). In (IV), the $\left[\mathrm{I}_{4}\right]^{2-}$ ion is nearly linear $\left[\mathrm{I} 2^{\mathrm{i}}-\mathrm{I} 2-\mathrm{I} 1=174.992(16)^{\circ}\right.$; symmetry code: $(\mathrm{i})-x-1,-y+1$, $-z+2$ ] and the I-to-I bonding is asymmetric, with a short central bond $\left[\mathrm{I} 2-\mathrm{I} 2^{\mathrm{i}}=2.7623(6) \AA\right]$ and two longer terminal bonds [I1-I2 = 3.3647 (4) $\mathrm{A}]$. Despite the large difference, both I-to-I distances are within the range routinely considered to denote bonding interactions and this is a fairly typical geometry for the $\left[\mathrm{I}_{4}\right]^{2-}$ ion (Svensson \& Kloo, 2003; Pan \& Englert, 2014). The $\left[\mathrm{I}_{4}\right]^{2-}$ ion lies on a crystallographic inversion centre; the similarity of the structure to the solvated chloride species is possible as the central $\mathrm{I}_{2}$ unit occupies the

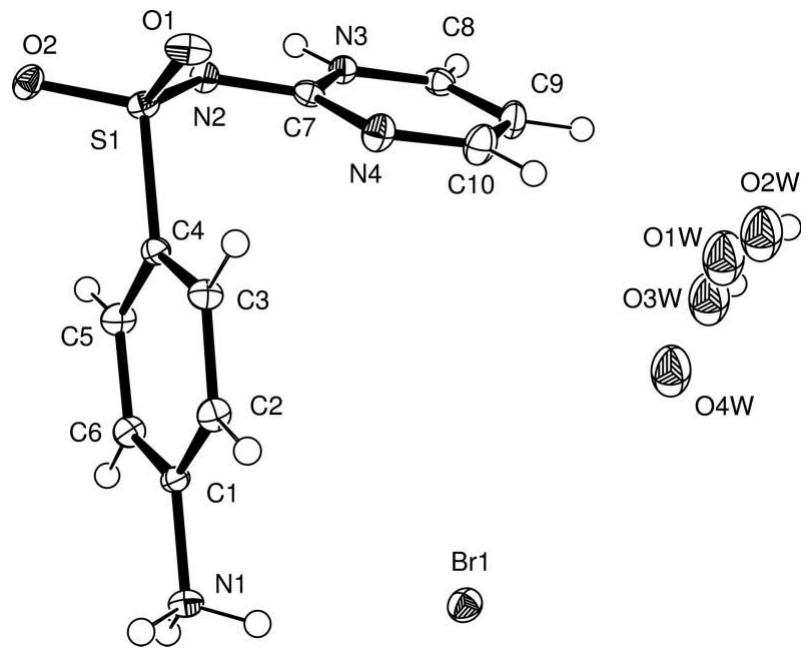

Figure 4

The molecular structure of (III), with non-H atoms shown as $50 \%$ probability displacement ellipsoids. $\mathrm{O} 1 W, \mathrm{O} 2 W, \mathrm{O} 3 W$ and $\mathrm{O} 4 W$ represent the partially occupied sites used to model a water molecule that is disordered in a channel parallel to the crystallographic $b$ axis.
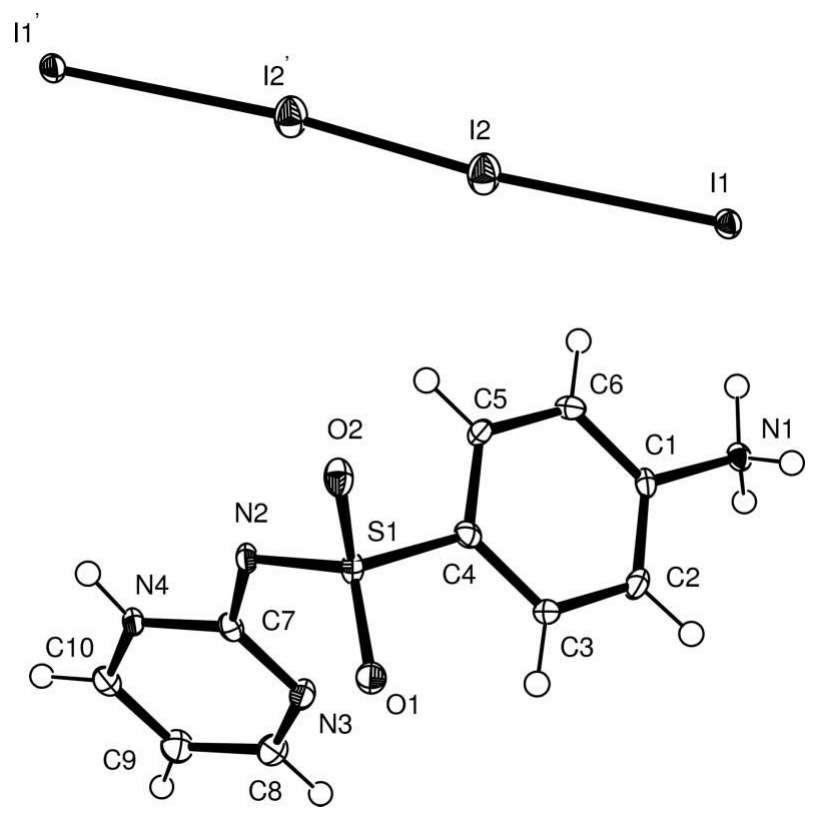

Figure 5

The molecular structure of (IV), with non-H atoms shown as $50 \%$ probability displacement ellipsoids. The contents of the asymmetric unit have been expanded to show a complete $\left[\mathrm{I}_{4}\right]^{2-}$ ion. [Symmetry code: (i) $-x-1,-y+1,-z+2$.]

space taken by solvent in the chloride structures, whilst the terminal I atoms take up the positions occupied by the chloride ions. Note that the water molecules in the hydrobromide are disordered in channels parallel to the crystallographic $b$ direction; such disorder is often seen for other channel hydrates. In bromide monohydrate (III), the water molecule has been modelled as split over four sites and these have been restrained to have a total occupancy of $1 . \mathrm{H}$ atoms

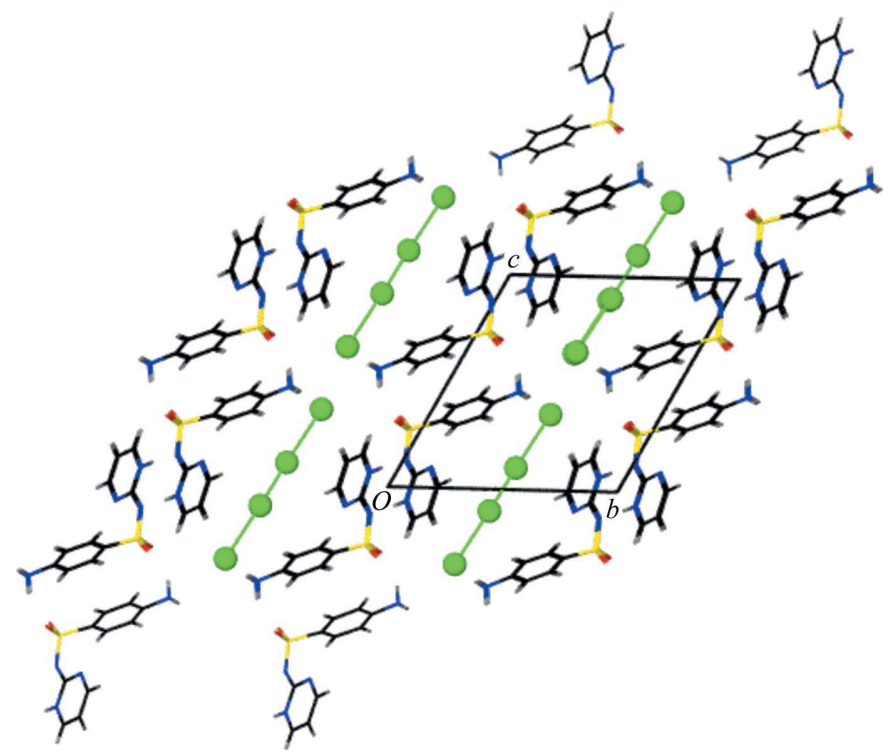

Figure 6

Packing diagram for (IV), viewed down the $a$ axis. The central two I atoms of each $\mathrm{I}_{4}$ unit occupy equivalent structural sites to the solvent molecules in the chloride solvate structures. 


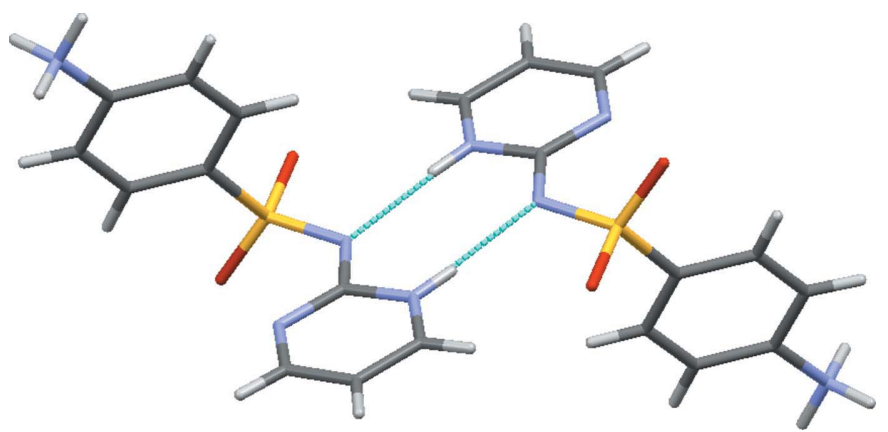

Figure 7

The centrosymmetric dimer which is robust enough to be found in all structures discussed herein. This example is drawn from structure (VI) and here the two cations are related by the symmetry operation $(-x+1$, $-y,-z+2)$.

could only be included for one of these sites, i.e. $\mathrm{O} 1 \mathrm{~W}$, which is the water O-atom site with the largest occupancy [45.3 (10)\%].

All these halide structures feature protonation at one of the pyrimidine ring $\mathrm{N}$ atoms and at the aniline group. This allows centrosymmetric hydrogen-bonded dimers to form which can be described using the $R_{2}^{2}(8)$ graph set (Bernstein et al., 1995) (Fig. 7). For the ethylene glycol solvate of the hydrochloride salt, Pan et al. (2013) suggested that the unusual protonation of the aniline group was adopted as alternatives would disturb this energetically favourable dimeric interaction. This argument is strengthened by the persistence of the same dimeric unit through the halide structures presented here and indeed by the existence of the same dimeric unit in the hydrated tetrafluoroborate, (V) (Fig. 8), and nitrate, (VI) (Fig. 9), salt structures. These two structures also retain the fundamental layering structure of the halide species; compare Fig. 3 with Fig. 10. The $-\mathrm{NH}_{3}{ }^{+}$group of all the halide species acts as a threefold hydrogen-bond donor. Two of the hydrogen bonds are to halide ions and thus link the cation layers to the anion layers, but the third hydrogen bond is formed with an $\mathrm{O}$ atom of the $-\mathrm{SO}_{2}$ - fragment. This third hydrogen bond links the cationic dimers into a one-dimensional chain (Fig. 11). Nitrate

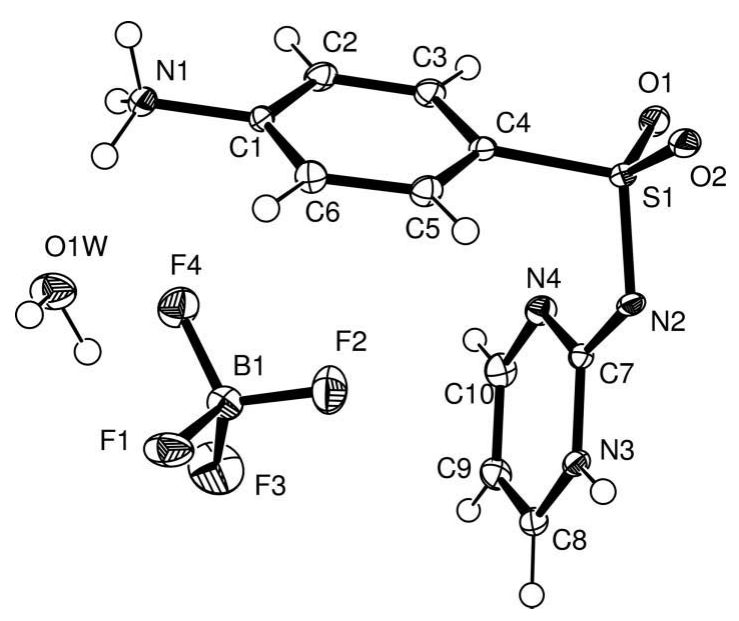

Figure 8

The molecular structure of $(\mathrm{V})$, with non-H atoms shown as $50 \%$ probability displacement ellipsoids.

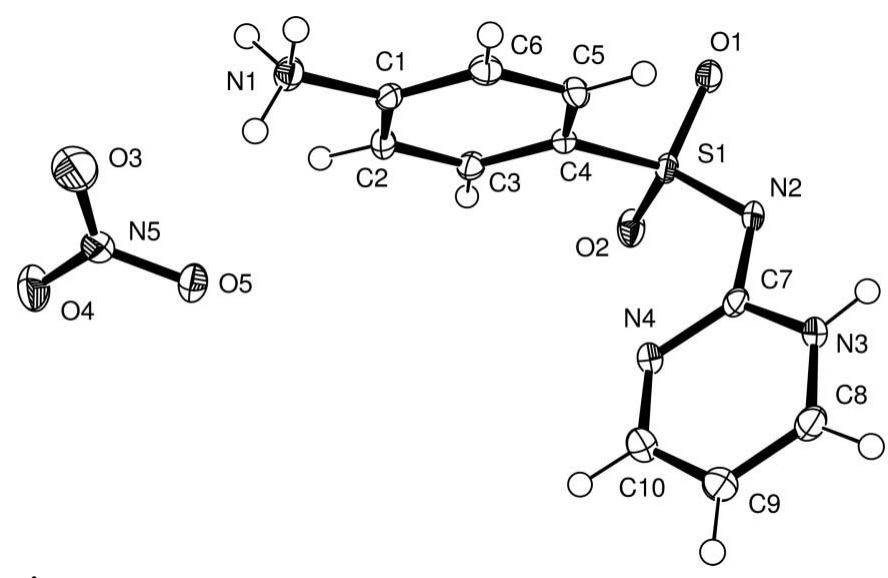

Figure 9

The molecular structure of (VI), with non- $\mathrm{H}$ atoms shown as $50 \%$ probability displacement ellipsoids.

structure (VI) is very similar, with the simple change that the $-\mathrm{NH}_{3}{ }^{+}$group forms bifurcated hydrogen bonds with $\mathrm{O}$ atoms of the anions, whilst the structure of the $\mathrm{BF}_{4}{ }^{-}$species $(\mathrm{V})$ differs slightly in that one $-\mathrm{NH}_{3}{ }^{+} \mathrm{H}$ atom, i.e. $\mathrm{H} 3 \mathrm{~N}$, forms a hydrogen bond with a water molecule acting as the acceptor. In all of structures (I)-(VI), this is the only direct sulfadiazineto-solvent hydrogen bond.

The final two structures both have sulfonate-based anions, viz. ethanesulfonate in (VII) (Fig. 12) and 4-hydroxybenzenesulfonate in (VIII) (Fig. 13), and both are structurally different from the other species. In the sulfonate salts, the sulfadiazine is protonated at the aniline and amide $\mathrm{N}$ atoms and not at either of the pyrimidine ring $\mathrm{N}$ atoms. Despite this difference in tautomeric form, the $R_{2}^{2}(8)$ dimer of cations motif

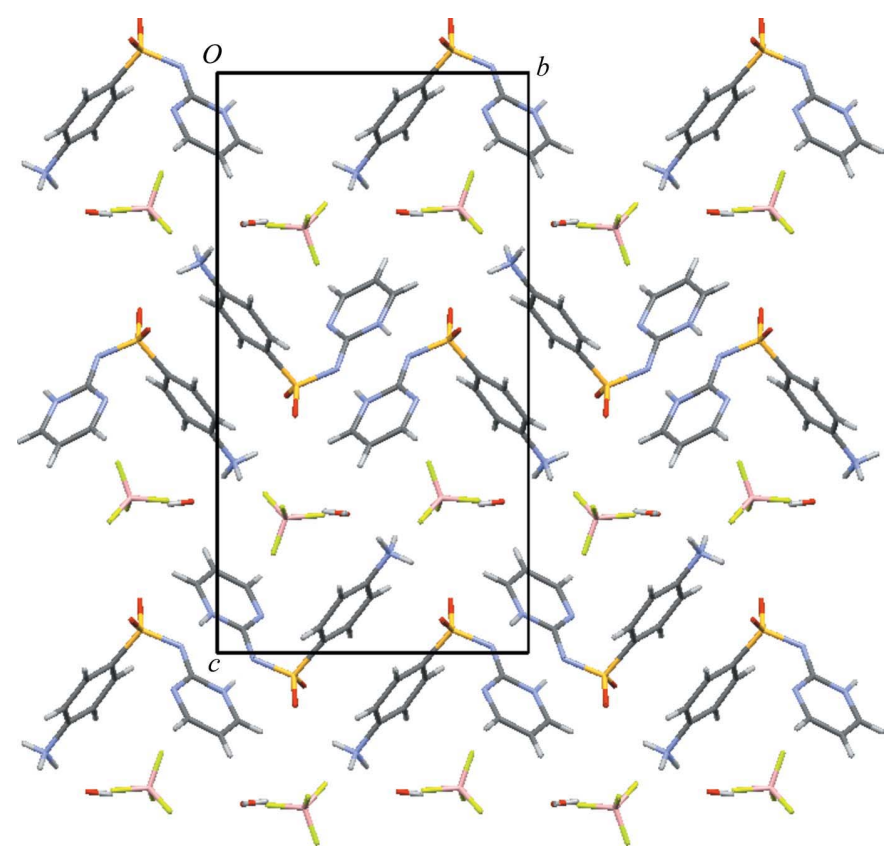

Figure 10

The packing of $(\mathrm{V})$, viewed down the $a$ axis. Although not part of the isostructural group formed by the chloride and $\left[\mathrm{I}_{4}\right]^{2-}$ salts, the main stuctural features of bilayers of $\mathrm{V}$-shaped cations separated by layers of anions and water molecules is retained. 


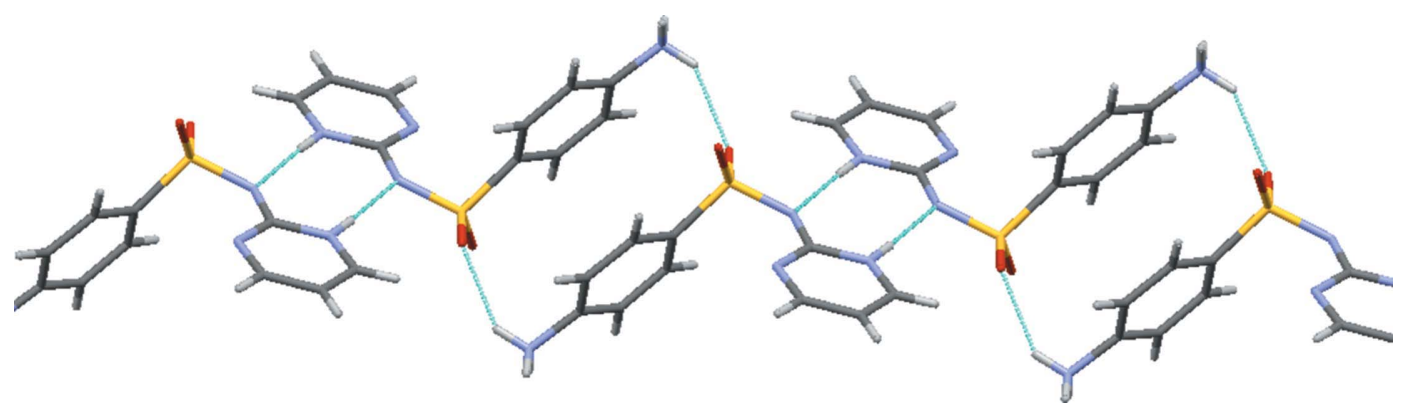

Figure 11

Part of the one-dimensional hydrogen-bonded chain of cations, drawn here for structure (II). The same supramolecular motif is found in structures (I)(VI). Here the view is down the $a$ axis and the chain extends along the [011] diagonal.

is retained. The dimeric structure is retained as the pyrimidine ring simply switches roles from hydrogen-bonding donor to hydrogen-bonding acceptor, whilst the amide group becomes the donor rather than the acceptor. Change to tautomeric form with retention of the same dimeric motif has also been described for related sulfonamides (Gelbrich et al., 2007) and indeed can be seen when comparing the structure of neutral sulfadiazine with that of its salt forms (Pan et al., 2013). In the latter case, change in tautomeric form was accompanied by change in molecular conformation. In particular, rotation about the $\mathrm{S}-\mathrm{C}$ bond varied by approximately $35^{\circ}$ between the amide-protonated species and the ring-protonated species. A similar division is not seen herein, with the dihedral angle between the planes defined by atoms N2/S1/C4 and the C1-C6 aromatic ring varying from $43.75(10)$ to $64.13(7)^{\circ}$ for compounds (I)-(VI) and being $51.44(14)$ and $60.12(11)^{\circ}$ for (VII) and (VIII), respectively. Elacqua et al. (2013) reported that neutral and anionic forms of sulfadiazine were differentiated by $\mathrm{S}-\mathrm{N}$ bond distances, with neutral species having distances of 1.61-1.65 $\AA$ and anions having distances of 1.56$1.60 \AA$. The cationic sulfadiazines (I)-(VI) have distances intermediate to these values [range 1.5941 (17)-1.6061 (12) A] ,

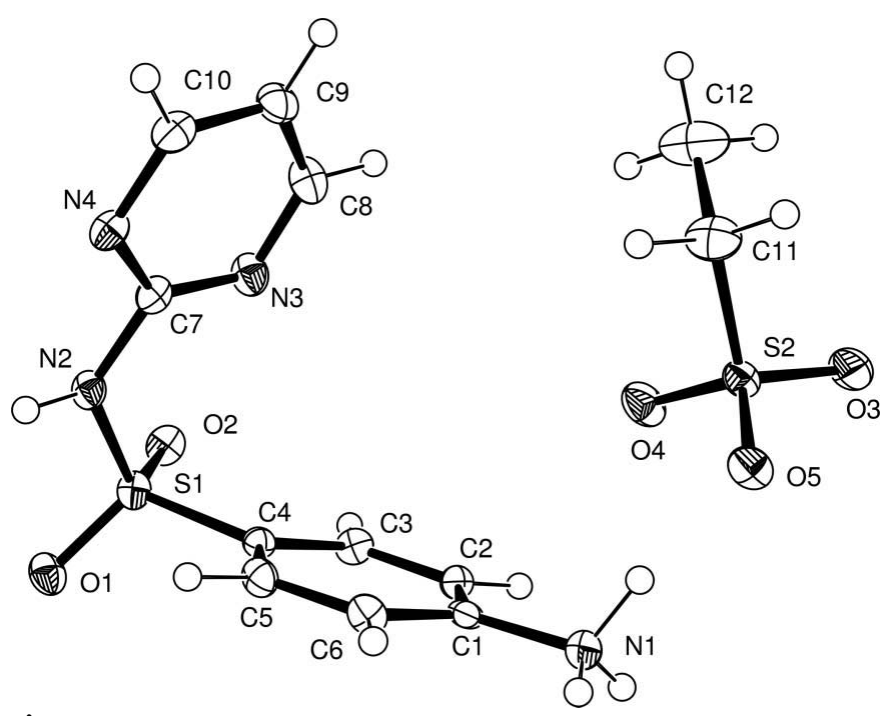

Figure 12

The molecular structure of (VII), with non-H atoms shown as $50 \%$ probability displacement ellipsoids. whilst the amide-protonated species (VII) and (VIII) have longer bond lengths $[1.633$ (3) and 1.6422 (19) $\AA$, respectively]. The adjacent $\mathrm{N}-\mathrm{C}$ bonds are also slightly longer for (VII) and (VIII) [1.388 (4) and 1.386 (3) ^, respectively] than they are for the ring-protonated species (I)-(VI) [range 1.337 (3)-1.347 (5) Å]. Structures (VII) and (VIII) also differ from the other sulfadiazine salts as the $-\mathrm{SO}_{2}-$ unit takes no part in hydrogen bonding other than with water and thus individual dimers do not connect through hydrogen bonding and so the polymeric motif described in Fig. 11 is absent. Instead, in ethanesulfonate salt (VII), the three $\mathrm{H}$ atoms of the $-\mathrm{NH}_{3}{ }^{+}$group all donate hydrogen bonds to $\mathrm{O}$ atoms of the sulfonate group and in the 4-hydroxybenzenesulfonate dihydrate (VIII) the $-\mathrm{NH}_{3}{ }^{+}$group acts as a hydrogen-bond donor to two water molecules and to one $\mathrm{O}$ atom of a sulfonate group. Although layered structures are retained, they are not the same as seen for the other species (Fig. 14). Only sulfonate-based anions appear to support sulfadiazine cations with the amide-protonated tautomeric form, but it is not obvious as to why this should be. $\mathrm{As} \mathrm{BF}_{4}{ }^{-}$is a relatively poor hydrogenbond acceptor, it may be that the tetrahedral shape of the sulfonate group together with its nature as a good and thus preferred hydrogen-bond acceptor is the key. However, whilst the structure of (VII), where ethanesulfonate forms multiple hydrogen-bonding interactions with the $\mathrm{RNH}_{3}{ }^{+}$group of the sulfadiazine cation may support such a connection, the

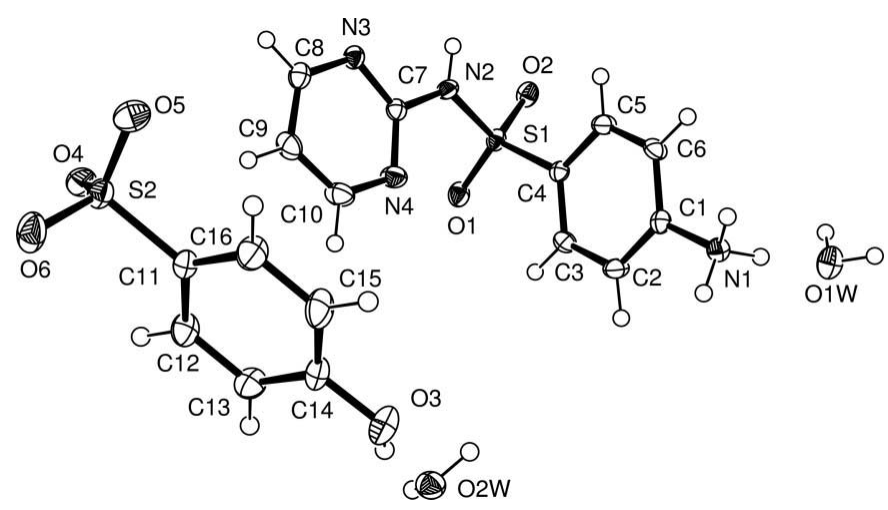

Figure 13

The molecular structure of (VIII), with non-H atoms shown as $50 \%$ probability displacement ellipsoids. 


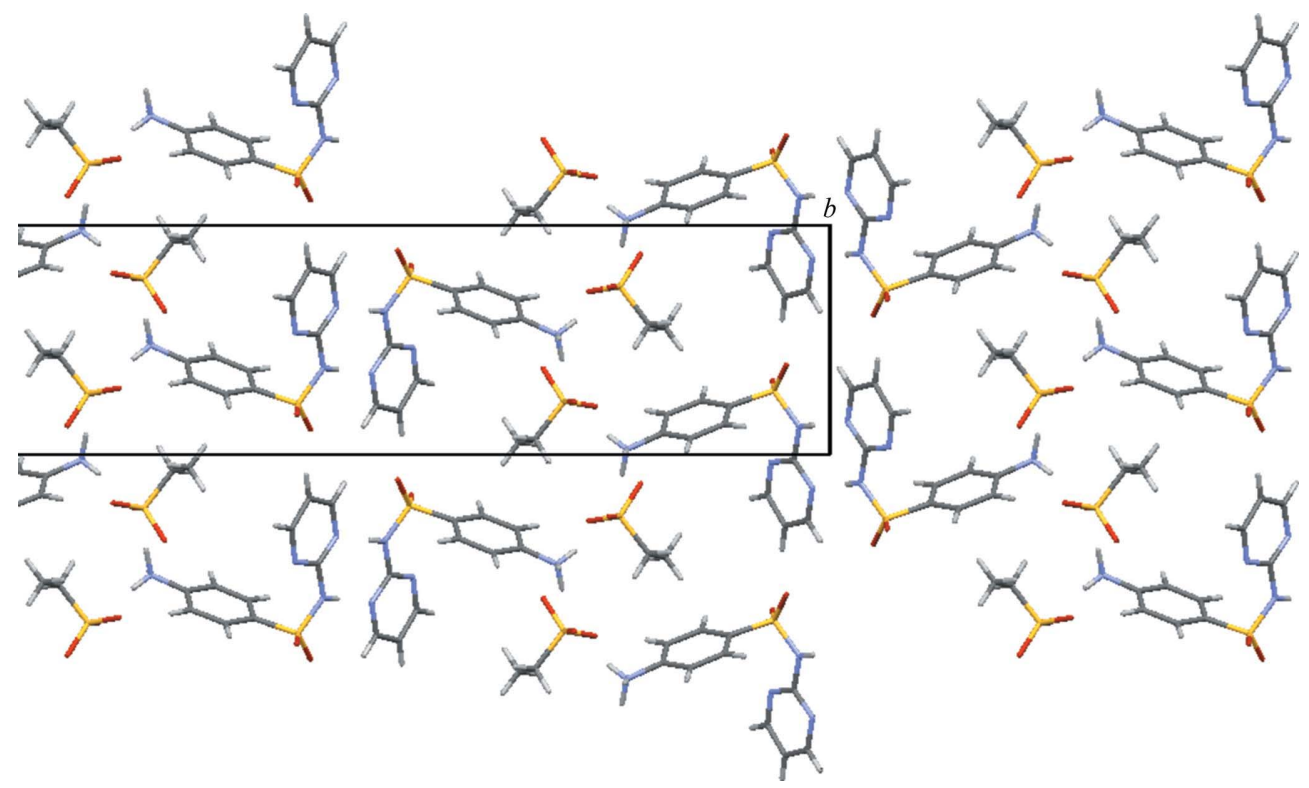

Figure 14

Packing diagram for (VII), viewed down the $a$ axis, showing the alternating layer structure.

structure of dihydrate (VIII), where the sulfonate group mostly interacts with water molecules, does not.

We thank the National Crystallography Service (University of Southampton) for the data collections on (I) and (II) (Cole \& Gale, 2012). CM wishes to thank the Nuffield Foundation for funding a research placement at the University of Strathclyde.

\section{References}

Allen, F. H. (2002). Acta Cryst. B58, 380-388.

Altomare, A., Cascarano, G., Giacovazzo, C., Guagliardi, A., Burla, M. C.,

Polidori, G. \& Camalli, M. (1994). J. Appl. Cryst. 27, 435.

Barbour, L. J. (2001). J. Supramol. Chem. 1, 189-191.

Bernstein, J., Davis, R. E., Shimoni, L. \& Chang, N.-L. (1995). Angew. Chem. Int. Ed. Engl. 34, 1555-1573.

Buist, A. R., Kennedy, A. R. \& Manzie, C. (2014). Acta Cryst. C70, 220-224.

Buist, A. R., Kennedy, A. R., Shankland, K., Shankland, N. \& Spillman, M. J. (2013). Cryst. Growth Des. 13, 5121-5127.

Clark, R. C. \& Reid, J. S. (1995). Acta Cryst. A51, 887-897.

Cole, S. J. \& Gale, P. A. (2012). Chem. Sci. 3, 683-689.

Cook, D. S. \& Turner, M. F. (1975). J. Chem. Soc. Perkin Trans. 2, pp. 10211025 .
Elacqua, E., Bucar, D.-K., Henry, R. F., Zhang, G. G. Z. \& MacGillivray, L. R. (2013). Cryst. Growth Des. 13, 393-403.

Farrugia, L. J. (2012). J. Appl. Cryst. 45, 849-854.

Fisher, N. M., Marsh, E. \& Lazova, R. (2003). J. Am. Acad. Dermatol. 49, 730732.

Gelbrich, T., Threlfall, T. L., Bingham, A. L. \& Hursthouse, M. B. (2007). Acta Cryst. C63, o323-0326.

Kokila, M. K., Puttaraja, Kulkarni, M. V. \& Thampi, S. (1995). Acta Cryst. C51, 333-336.

Lu, E., Rodriguez-Hornedo, N. \& Suryanarayanan, R. (2008). CrystEngComm, 10, 665-668.

Macrae, C. F., Bruno, I. J., Chisholm, J. A., Edgington, P. R., McCabe, P., Pidcock, E., Rodriguez-Monge, L., Taylor, R., van de Streek, J. \& Wood, P. A. (2008). J. Appl. Cryst. 41, 466-470.

Oxford Diffraction (2010). CrysAlis CCD and CrysAlis RED. Oxford Diffraction Ltd, Abingdon, Oxfordshire, England.

Pan, F. \& Englert, U. (2014). Cryst. Growth Des. 14, 1057-1066.

Pan, F., Wang, R. \& Englert, U. (2013). CrystEngComm, 15, 1164-1172.

Rigaku (2013). CrystalClear-SM Expert. Rigaku Corporation, Tokyo, Japan.

Sheldrick, G. M. (2008). Acta Cryst. A64, 112-122.

Smith, G. \& Wermuth, U. D. (2013a). Acta Cryst. C69, 538-543.

Smith, G. \& Wermuth, U. D. (2013b). Acta Cryst. E69, o472.

Stahl, P. H. \& Wermuth, C. G. (2008). Editors. Handbook of Pharmaceutical Salts: Properties, Selection and Use. Zurich: VHCA.

Svensson, P. H. \& Kloo, L. (2003). Chem. Rev. 103, 1649-1684.

Weclawik, M., Gagor, A., Piecha, A., Jakubas, R. \& Medycki, W. (2013). CrystEngComm, 15, 5633-5640. 


\section{supporting information}

Acta Cryst. (2014). C70, 900-907 [doi:10.1107/S2053229614018725]

\section{Eight salt forms of sulfadiazine}

\section{Amanda R. Buist, Lynn Dennany, Alan R. Kennedy, Craig Manzie, Katherine McPhie and Brandon Walker}

\section{Computing details}

Data collection: CrystalClear-SM Expert (Rigaku, 2013) for (I), (II); CrysAlis PRO (Oxford Diffraction, 2010) for (III), (IV), (V), (VI), (VII), (VIII). Cell refinement: CrystalClear-SM Expert (Rigaku, 2013) for (I), (II); CrysAlis PRO (Oxford Diffraction, 2010) for (III), (IV), (V), (VI), (VII), (VIII). Data reduction: CrystalClear-SM Expert (Rigaku, 2013) for (I), (II); CrysAlis PRO (Oxford Diffraction, 2010) for (III), (IV), (V), (VI), (VII), (VIII). Program(s) used to solve structure: SIR92 (Altomare et al., 1994) for (I), (II); SHELXS97 (Sheldrick, 2008) for (III), (IV), (V), (VI), (VII), (VIII). For all compounds, program(s) used to refine structure: SHELXL97 (Sheldrick, 2008). Molecular graphics: ORTEP-3 for Windows (Farrugia, 2012) and Mercury (Macrae et al., 2008) for (I), (II), (V), (VI), (VII); ORTEP-3 for Windows (Farrugia, 2012) for (III), (VIII); ORTEP-3 for Windows (Farrugia, 2012) and X-SEED (Barbour, 2001) for (IV). For all compounds, software used to prepare material for publication: SHELXL97 (Sheldrick, 2008).

\section{(I) 2-\{[(4-Azaniumylphenyl)sulfonyl]azanidyl\}pyrimidinium chloride monohydrate}

Crystal data

$\mathrm{C}_{10} \mathrm{H}_{11} \mathrm{~N}_{4} \mathrm{O}_{2} \mathrm{~S}^{+} \cdot \mathrm{Cl}^{-} \cdot \mathrm{H}_{2} \mathrm{O}$

$M_{r}=304.75$

Triclinic, $P \overline{1}$

Hall symbol: -P 1

$a=5.4118(4) \AA$

$b=11.5632(8) \AA$

$c=11.7430(8) \AA$

$\alpha=109.462(7)^{\circ}$

$\beta=94.399(6)^{\circ}$

$\gamma=102.509(7)^{\circ}$

$V=667.51(8) \AA^{3}$

Data collection

Rigaku Saturn724+ (2x2 bin mode) diffractometer

Radiation source: fine-focus sealed tube Mirrors monochromator Detector resolution: 28.5714 pixels $\mathrm{mm}^{-1}$ profile data from $\omega$-scans Absorption correction: multi-scan (CrystalClear-SM Expert; Rigaku, 2013)

$T_{\min }=0.897, T_{\max }=1.000$
$Z=2$

$F(000)=316$

$D_{\mathrm{x}}=1.516 \mathrm{Mg} \mathrm{m}^{-3}$

Mo $K \alpha$ radiation, $\lambda=0.71073 \AA$

Cell parameters from 56264 reflections

$\theta=3.1-27.5^{\circ}$

$\mu=0.45 \mathrm{~mm}^{-1}$

$T=100 \mathrm{~K}$

Plate, yellow

$0.06 \times 0.02 \times 0.01 \mathrm{~mm}$

8329 measured reflections

3000 independent reflections

2752 reflections with $I>2 \sigma(I)$

$R_{\text {int }}=0.025$

$\theta_{\text {max }}=27.5^{\circ}, \theta_{\min }=3.1^{\circ}$

$h=-7 \rightarrow 7$

$k=-14 \rightarrow 14$

$l=-15 \rightarrow 14$ 


\section{supporting information}

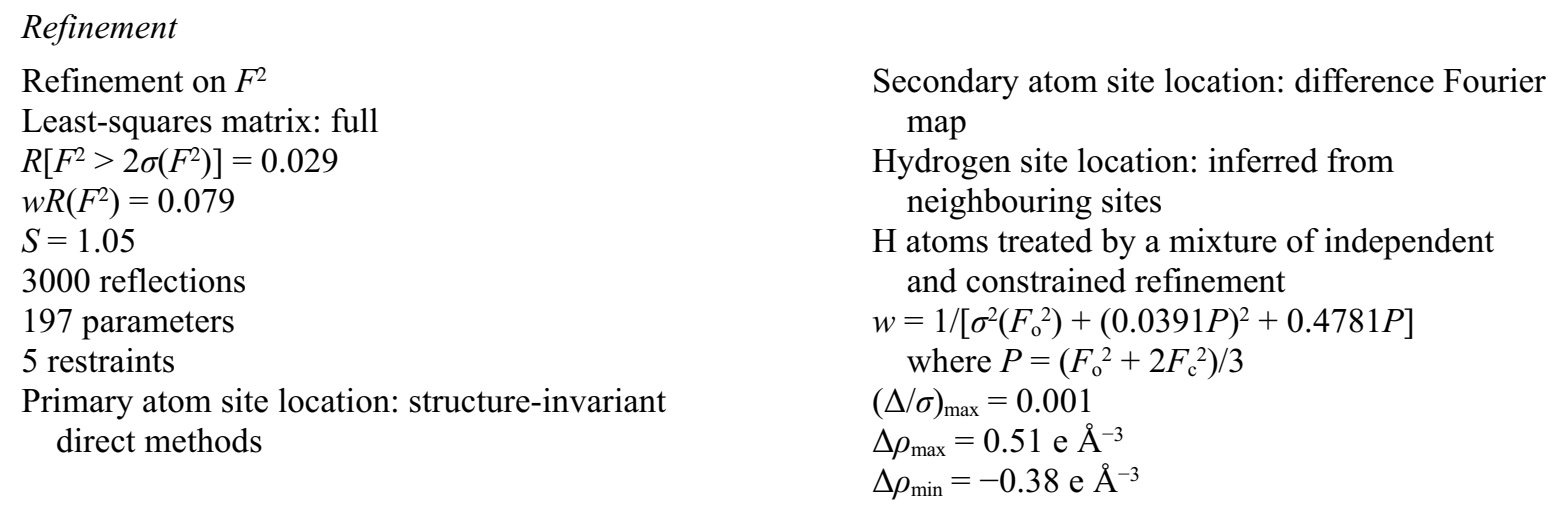

Special details

Experimental. NCS collection 2013ncs0582

Geometry. All s.u.'s (except the s.u. in the dihedral angle between two l.s. planes) are estimated using the full covariance matrix. The cell s.u.'s are taken into account individually in the estimation of s.u.'s in distances, angles and torsion angles; correlations between s.u.'s in cell parameters are only used when they are defined by crystal symmetry. An approximate (isotropic) treatment of cell s.u.'s is used for estimating s.u.'s involving l.s. planes.

Refinement. One $\mathrm{H}$ atom of water molecule positioned as disordered over two sites.

Refinement of $F^{2}$ against ALL reflections. The weighted $R$-factor $w R$ and goodness of fit $S$ are based on $F^{2}$, conventional $R$-factors $R$ are based on $F$, with $F$ set to zero for negative $F^{2}$. The threshold expression of $F^{2}>2 \sigma\left(F^{2}\right)$ is used only for calculating $R$-factors(gt) etc. and is not relevant to the choice of reflections for refinement. $R$-factors based on $F^{2}$ are statistically about twice as large as those based on $F$, and $R$ - factors based on ALL data will be even larger.

Fractional atomic coordinates and isotropic or equivalent isotropic displacement parameters $\left(\AA^{2}\right)$

\begin{tabular}{|c|c|c|c|c|c|}
\hline & $x$ & $y$ & $z$ & $U_{\text {iso }} * / U_{\text {eq }}$ & Occ. $(<1)$ \\
\hline $\mathrm{C} 11$ & $1.26745(6)$ & $1.00601(3)$ & $0.85165(3)$ & $0.01651(10)$ & \\
\hline $\mathrm{S} 1$ & $0.16642(6)$ & $0.43185(3)$ & $0.23791(3)$ & $0.01025(9)$ & \\
\hline $\mathrm{O} 1$ & $-0.07441(19)$ & $0.33435(9)$ & $0.19198(9)$ & $0.0140(2)$ & \\
\hline $\mathrm{O} 2$ & $0.3914(2)$ & $0.39099(10)$ & $0.20278(9)$ & $0.0155(2)$ & \\
\hline $\mathrm{O} 1 \mathrm{~W}$ & $1.2540(3)$ & $0.98671(16)$ & $0.55066(15)$ & $0.0458(4)$ & \\
\hline $\mathrm{H} 1 \mathrm{~W}$ & $1.277(6)$ & $1.013(3)$ & $0.6311(9)$ & $0.069^{*}$ & \\
\hline $\mathrm{H} 2 \mathrm{~W}$ & $1.411(4)$ & $1.019(5)$ & $0.541(4)$ & $0.069 *$ & 0.50 \\
\hline $\mathrm{H} 3 \mathrm{~W}$ & $1.122(8)$ & $1.017(5)$ & $0.540(4)$ & $0.069 *$ & 0.50 \\
\hline N1 & $0.1521(2)$ & $0.84971(11)$ & $0.04582(11)$ & $0.0129(2)$ & \\
\hline N2 & $0.1647(2)$ & $0.48996(11)$ & $0.38226(10)$ & $0.0119(2)$ & \\
\hline N3 & $0.3056(2)$ & $0.64473(11)$ & $0.57102(11)$ & $0.0117(2)$ & \\
\hline N4 & $0.5611(2)$ & $0.64491(12)$ & $0.41730(11)$ & $0.0146(2)$ & \\
\hline $\mathrm{C} 1$ & $0.1482(3)$ & $0.74771(13)$ & $0.09422(12)$ & $0.0111(3)$ & \\
\hline $\mathrm{C} 2$ & 0.3230 & $0.67568(13)$ & $0.06079(12)$ & $0.0128(3)$ & \\
\hline $\mathrm{H} 2$ & 0.4377 & 0.6913 & 0.0071 & $0.015^{*}$ & \\
\hline $\mathrm{C} 3$ & 0.3268 & $0.58038(13)$ & $0.10742(12)$ & $0.0122(3)$ & \\
\hline H3 & 0.4490 & 0.5318 & 0.0881 & $0.015^{*}$ & \\
\hline $\mathrm{C} 4$ & $0.1518(3)$ & $0.55606(12)$ & $0.18235(12)$ & $0.0101(3)$ & \\
\hline $\mathrm{C} 5$ & $-0.0265(3)$ & $0.62670(13)$ & $0.21399(13)$ & 0.0133 & \\
\hline H5 & -0.1467 & 0.6083 & 0.2644 & $0.016^{*}$ & \\
\hline C6 & $-0.0256(3)$ & $0.72526(13)$ & $0.17032(13)$ & 0.0133 & \\
\hline H6 & -0.1426 & 0.7764 & 0.1924 & $0.016^{*}$ & \\
\hline
\end{tabular}




\begin{tabular}{lllll} 
C7 & $0.3486(3)$ & $0.59269(13)$ & $0.45379(12)$ & $0.0111(3)$ \\
C8 & $0.4704(3)$ & $0.74805(13)$ & $0.65421(13)$ & $0.0141(3)$ \\
H8 & 0.4354 & 0.7823 & 0.7350 & $0.017^{*}$ \\
C9 & $0.6903(3)$ & $0.80354(14)$ & $0.62089(14)$ & $0.0167(3)$ \\
H9 & 0.8121 & 0.8765 & 0.6769 & $0.020^{*}$ \\
C10 & $0.7250(3)$ & $0.74683(14)$ & $0.50033(14)$ & $0.0169(3)$ \\
H10 & 0.8763 & 0.7835 & 0.4756 & $0.020^{*}$ \\
H1N & $0.310(4)$ & $0.906(2)$ & $0.070(2)$ & $0.029(5)^{*}$ \\
H2N & $0.034(4)$ & $0.8936(19)$ & $0.0730(18)$ & $0.022(5)^{*}$ \\
H3N & $0.118(4)$ & $0.814(2)$ & $-0.038(2)$ & $0.027(5)^{*}$ \\
H4N & $0.162(4)$ & $0.6042(19)$ & $0.5895(18)$ & $0.023(5)^{*}$ \\
\hline
\end{tabular}

Atomic displacement parameters $\left(\AA^{2}\right)$

\begin{tabular}{lllllll}
\hline & $U^{11}$ & $U^{22}$ & $U^{33}$ & $U^{12}$ & $U^{13}$ & $U^{23}$ \\
\hline C11 & $0.01210(17)$ & $0.01286(17)$ & $0.02310(19)$ & $0.00332(12)$ & $0.00203(13)$ & $0.00472(13)$ \\
S1 & $0.01084(17)$ & $0.01154(16)$ & $0.00981(16)$ & $0.00382(12)$ & $0.00344(11)$ & $0.00467(12)$ \\
O1 & $0.0147(5)$ & $0.0131(5)$ & $0.0124(5)$ & $0.0010(4)$ & $0.0021(4)$ & $0.0039(4)$ \\
O2 & $0.0162(5)$ & $0.0198(5)$ & $0.0164(5)$ & $0.0102(4)$ & $0.0081(4)$ & $0.0095(4)$ \\
O1W & $0.0516(10)$ & $0.0450(9)$ & $0.0401(8)$ & $0.0006(8)$ & $0.0041(7)$ & $0.0226(7)$ \\
N1 & $0.0132(6)$ & $0.0118(6)$ & $0.0143(6)$ & $0.0032(5)$ & $0.0023(4)$ & $0.0055(5)$ \\
N2 & $0.0113(5)$ & $0.0143(6)$ & $0.0097(5)$ & $0.0021(4)$ & $0.0028(4)$ & $0.0043(4)$ \\
N3 & $0.0108(6)$ & $0.0138(5)$ & $0.0115(5)$ & $0.0032(4)$ & $0.0033(4)$ & $0.0056(4)$ \\
N4 & $0.0122(6)$ & $0.0174(6)$ & $0.0147(6)$ & $0.0023(5)$ & $0.0039(4)$ & $0.0070(5)$ \\
C1 & $0.0113(6)$ & $0.0102(6)$ & $0.0104(6)$ & $0.0012(5)$ & $-0.0002(5)$ & $0.0035(5)$ \\
C2 & $0.0116(6)$ & $0.0153(6)$ & $0.0120(6)$ & $0.0032(5)$ & $0.0041(5)$ & $0.0053(5)$ \\
C3 & $0.0105(6)$ & $0.0143(6)$ & $0.0128(6)$ & $0.0054(5)$ & $0.0040(5)$ & $0.0045(5)$ \\
C4 & $0.0099(6)$ & $0.0105(6)$ & $0.0096(6)$ & $0.0019(5)$ & $0.0010(5)$ & $0.0040(5)$ \\
C5 & $0.0119(6)$ & $0.0158(6)$ & $0.0133(6)$ & $0.0038(5)$ & $0.0049(5)$ & $0.0057(5)$ \\
C6 & $0.0118(6)$ & $0.0146(6)$ & $0.0146(6)$ & $0.0055(5)$ & $0.0039(5)$ & $0.0048(5)$ \\
C7 & $0.0105(6)$ & $0.0131(6)$ & $0.0121(6)$ & $0.0049(5)$ & $0.0025(5)$ & $0.0064(5)$ \\
C8 & $0.0166(7)$ & $0.0129(6)$ & $0.0131(6)$ & $0.0048(5)$ & $0.0018(5)$ & $0.0046(5)$ \\
C9 & $0.0158(7)$ & $0.0139(6)$ & $0.0172(7)$ & $-0.0001(5)$ & $0.0002(5)$ & $0.0044(5)$ \\
C10 & $0.0132(7)$ & $0.0178(7)$ & $0.0198(7)$ & $0.0010(5)$ & $0.0037(5)$ & $0.0087(6)$ \\
& & & & & & \\
\hline
\end{tabular}

Geometric parameters $\left(A,{ }^{\circ}\right)$

\begin{tabular}{llll}
\hline $\mathrm{S} 1-\mathrm{O} 2$ & $1.4397(10)$ & $\mathrm{N} 4-\mathrm{C} 7$ & $1.3484(18)$ \\
$\mathrm{S} 1-\mathrm{O} 1$ & $1.4530(10)$ & $\mathrm{C} 1-\mathrm{C} 2$ & $1.387(2)$ \\
$\mathrm{S} 1-\mathrm{N} 2$ & $1.6034(12)$ & $\mathrm{C} 1-\mathrm{C} 6$ & $1.3886(19)$ \\
$\mathrm{S} 1-\mathrm{C} 4$ & $1.7777(14)$ & $\mathrm{C} 2-\mathrm{C} 3$ & $1.387(2)$ \\
$\mathrm{O} 1 \mathrm{~W}-\mathrm{H} 1 \mathrm{~W}$ & $0.880(10)$ & $\mathrm{C} 2-\mathrm{H} 2$ & 0.9500 \\
$\mathrm{O} 1 \mathrm{~W}-\mathrm{H} 2 \mathrm{~W}$ & $0.881(10)$ & $\mathrm{C} 3-\mathrm{C} 4$ & $1.3886(18)$ \\
$\mathrm{O} 1 \mathrm{~W}-\mathrm{H} 3 \mathrm{~W}$ & $0.882(10)$ & $\mathrm{C} 3-\mathrm{H} 3$ & 0.9500 \\
$\mathrm{~N} 1-\mathrm{C} 1$ & $1.4671(17)$ & $\mathrm{C} 4-\mathrm{C} 5$ & $1.3893(19)$ \\
$\mathrm{N} 1-\mathrm{H} 2 \mathrm{~N}$ & $0.91(2)$ & $\mathrm{C} 5-\mathrm{C} 6$ & $1.396(2)$ \\
$\mathrm{N} 1-\mathrm{H} 3 \mathrm{~N}$ & $0.91(2)$ & $\mathrm{C} 5-\mathrm{H} 5$ & 0.9500 \\
$\mathrm{~N} 1-\mathrm{H} 1 \mathrm{~N}$ & $0.91(2)$ & $\mathrm{C} 6-\mathrm{H} 6$ & 0.9500
\end{tabular}




\begin{tabular}{|c|c|c|c|}
\hline $\mathrm{N} 2-\mathrm{C} 7$ & $1.3421(18)$ & $\mathrm{C} 8-\mathrm{C} 9$ & $1.374(2)$ \\
\hline $\mathrm{N} 3-\mathrm{C} 8$ & $1.3431(19)$ & $\mathrm{C} 8-\mathrm{H} 8$ & 0.9500 \\
\hline N3-C7 & $1.3670(18)$ & $\mathrm{C} 9-\mathrm{C} 10$ & $1.394(2)$ \\
\hline $\mathrm{N} 3-\mathrm{H} 4 \mathrm{~N}$ & $0.90(2)$ & C9- $\mathrm{H} 9$ & 0.9500 \\
\hline $\mathrm{N} 4-\mathrm{C} 10$ & 1.3324 (19) & $\mathrm{C} 10-\mathrm{H} 10$ & 0.9500 \\
\hline $\mathrm{O} 2-\mathrm{S} 1-\mathrm{O} 1$ & $115.44(6)$ & $\mathrm{C} 3-\mathrm{C} 2-\mathrm{H} 2$ & 120.7 \\
\hline $\mathrm{O} 2-\mathrm{S} 1-\mathrm{N} 2$ & $115.82(6)$ & $\mathrm{C} 2-\mathrm{C} 3-\mathrm{C} 4$ & $119.88(13)$ \\
\hline $\mathrm{O} 1-\mathrm{S} 1-\mathrm{N} 2$ & $103.55(6)$ & $\mathrm{C} 2-\mathrm{C} 3-\mathrm{H} 3$ & 120.1 \\
\hline $\mathrm{O} 2-\mathrm{S} 1-\mathrm{C} 4$ & $106.80(6)$ & $\mathrm{C} 4-\mathrm{C} 3-\mathrm{H} 3$ & 120.1 \\
\hline $\mathrm{O} 1-\mathrm{S} 1-\mathrm{C} 4$ & $108.83(6)$ & $\mathrm{C} 3-\mathrm{C} 4-\mathrm{C} 5$ & $121.48(13)$ \\
\hline $\mathrm{N} 2-\mathrm{S} 1-\mathrm{C} 4$ & $105.90(6)$ & $\mathrm{C} 3-\mathrm{C} 4-\mathrm{S} 1$ & $117.44(11)$ \\
\hline $\mathrm{H} 1 \mathrm{~W}-\mathrm{O} 1 \mathrm{~W}-\mathrm{H} 2 \mathrm{~W}$ & $99(2)$ & $\mathrm{C} 5-\mathrm{C} 4-\mathrm{S} 1$ & $121.08(10)$ \\
\hline $\mathrm{H} 1 \mathrm{~W}-\mathrm{O} 1 \mathrm{~W}-\mathrm{H} 3 \mathrm{~W}$ & $98(2)$ & $\mathrm{C} 4-\mathrm{C} 5-\mathrm{C} 6$ & $118.77(13)$ \\
\hline $\mathrm{H} 2 \mathrm{~W}-\mathrm{O} 1 \mathrm{~W}-\mathrm{H} 3 \mathrm{~W}$ & $125(6)$ & $\mathrm{C} 4-\mathrm{C} 5-\mathrm{H} 5$ & 120.6 \\
\hline $\mathrm{C} 1-\mathrm{N} 1-\mathrm{H} 2 \mathrm{~N}$ & $112.3(13)$ & $\mathrm{C} 6-\mathrm{C} 5-\mathrm{H} 5$ & 120.6 \\
\hline $\mathrm{C} 1-\mathrm{N} 1-\mathrm{H} 3 \mathrm{~N}$ & $108.3(14)$ & $\mathrm{C} 1-\mathrm{C} 6-\mathrm{C} 5$ & $119.24(13)$ \\
\hline $\mathrm{H} 2 \mathrm{~N}-\mathrm{N} 1-\mathrm{H} 3 \mathrm{~N}$ & $109.1(18)$ & $\mathrm{C} 1-\mathrm{C} 6-\mathrm{H} 6$ & 120.4 \\
\hline $\mathrm{C} 1-\mathrm{N} 1-\mathrm{H} 1 \mathrm{~N}$ & $109.2(14)$ & $\mathrm{C} 5-\mathrm{C} 6-\mathrm{H} 6$ & 120.4 \\
\hline $\mathrm{H} 2 \mathrm{~N}-\mathrm{N} 1-\mathrm{H} 1 \mathrm{~N}$ & $107.9(18)$ & $\mathrm{N} 2-\mathrm{C} 7-\mathrm{N} 4$ & $124.87(13)$ \\
\hline $\mathrm{H} 3 \mathrm{~N}-\mathrm{N} 1-\mathrm{H} 1 \mathrm{~N}$ & $110.1(18)$ & $\mathrm{N} 2-\mathrm{C} 7-\mathrm{N} 3$ & $114.71(12)$ \\
\hline $\mathrm{C} 7-\mathrm{N} 2-\mathrm{S} 1$ & $120.21(10)$ & $\mathrm{N} 4-\mathrm{C} 7-\mathrm{N} 3$ & $120.42(13)$ \\
\hline $\mathrm{C} 8-\mathrm{N} 3-\mathrm{C} 7$ & $122.26(12)$ & $\mathrm{N} 3-\mathrm{C} 8-\mathrm{C} 9$ & $119.12(13)$ \\
\hline $\mathrm{C} 8-\mathrm{N} 3-\mathrm{H} 4 \mathrm{~N}$ & $122.0(13)$ & $\mathrm{N} 3-\mathrm{C} 8-\mathrm{H} 8$ & 120.4 \\
\hline $\mathrm{C} 7-\mathrm{N} 3-\mathrm{H} 4 \mathrm{~N}$ & $115.7(13)$ & $\mathrm{C} 9-\mathrm{C} 8-\mathrm{H} 8$ & 120.4 \\
\hline $\mathrm{C} 10-\mathrm{N} 4-\mathrm{C} 7$ & $117.14(13)$ & $\mathrm{C} 8-\mathrm{C} 9-\mathrm{C} 10$ & $116.37(13)$ \\
\hline $\mathrm{C} 2-\mathrm{C} 1-\mathrm{C} 6$ & $121.98(13)$ & $\mathrm{C} 8-\mathrm{C} 9-\mathrm{H} 9$ & 121.8 \\
\hline $\mathrm{C} 2-\mathrm{C} 1-\mathrm{N} 1$ & $117.47(12)$ & $\mathrm{C} 10-\mathrm{C} 9-\mathrm{H} 9$ & 121.8 \\
\hline $\mathrm{C} 6-\mathrm{C} 1-\mathrm{N} 1$ & $120.55(12)$ & $\mathrm{N} 4-\mathrm{C} 10-\mathrm{C} 9$ & $124.70(13)$ \\
\hline $\mathrm{C} 1-\mathrm{C} 2-\mathrm{C} 3$ & $118.60(12)$ & $\mathrm{N} 4-\mathrm{C} 10-\mathrm{H} 10$ & 117.7 \\
\hline $\mathrm{C} 1-\mathrm{C} 2-\mathrm{H} 2$ & 120.7 & $\mathrm{C} 9-\mathrm{C} 10-\mathrm{H} 10$ & 117.7 \\
\hline $\mathrm{O} 2-\mathrm{S} 1-\mathrm{N} 2-\mathrm{C} 7$ & $59.45(13)$ & $\mathrm{S} 1-\mathrm{C} 4-\mathrm{C} 5-\mathrm{C} 6$ & $178.43(10)$ \\
\hline $\mathrm{O} 1-\mathrm{S} 1-\mathrm{N} 2-\mathrm{C} 7$ & $-173.14(11)$ & $\mathrm{C} 2-\mathrm{C} 1-\mathrm{C} 6-\mathrm{C} 5$ & $-0.5(2)$ \\
\hline $\mathrm{C} 4-\mathrm{S} 1-\mathrm{N} 2-\mathrm{C} 7$ & $-58.69(12)$ & $\mathrm{N} 1-\mathrm{C} 1-\mathrm{C} 6-\mathrm{C} 5$ & $179.38(12)$ \\
\hline $\mathrm{C} 6-\mathrm{C} 1-\mathrm{C} 2-\mathrm{C} 3$ & $-1.5(2)$ & $\mathrm{C} 4-\mathrm{C} 5-\mathrm{C} 6-\mathrm{C} 1$ & $1.7(2)$ \\
\hline $\mathrm{N} 1-\mathrm{C} 1-\mathrm{C} 2-\mathrm{C} 3$ & $178.65(12)$ & $\mathrm{S} 1-\mathrm{N} 2-\mathrm{C} 7-\mathrm{N} 4$ & $-9.0(2)$ \\
\hline $\mathrm{C} 1-\mathrm{C} 2-\mathrm{C} 3-\mathrm{C} 4$ & $2.2(2)$ & $\mathrm{S} 1-\mathrm{N} 2-\mathrm{C} 7-\mathrm{N} 3$ & $170.47(10)$ \\
\hline $\mathrm{C} 2-\mathrm{C} 3-\mathrm{C} 4-\mathrm{C} 5$ & $-1.1(2)$ & $\mathrm{C} 10-\mathrm{N} 4-\mathrm{C} 7-\mathrm{N} 2$ & $-179.79(13)$ \\
\hline $\mathrm{C} 2-\mathrm{C} 3-\mathrm{C} 4-\mathrm{S} 1$ & $179.56(10)$ & $\mathrm{C} 10-\mathrm{N} 4-\mathrm{C} 7-\mathrm{N} 3$ & $0.8(2)$ \\
\hline $\mathrm{O} 2-\mathrm{S} 1-\mathrm{C} 4-\mathrm{C} 3$ & $7.27(13)$ & $\mathrm{C} 8-\mathrm{N} 3-\mathrm{C} 7-\mathrm{N} 2$ & $-179.80(13)$ \\
\hline $\mathrm{O} 1-\mathrm{S} 1-\mathrm{C} 4-\mathrm{C} 3$ & $-117.98(11)$ & $\mathrm{C} 8-\mathrm{N} 3-\mathrm{C} 7-\mathrm{N} 4$ & $-0.3(2)$ \\
\hline $\mathrm{N} 2-\mathrm{S} 1-\mathrm{C} 4-\mathrm{C} 3$ & $131.25(11)$ & $\mathrm{C} 7-\mathrm{N} 3-\mathrm{C} 8-\mathrm{C} 9$ & $-0.2(2)$ \\
\hline $\mathrm{O} 2-\mathrm{S} 1-\mathrm{C} 4-\mathrm{C} 5$ & $-172.08(11)$ & $\mathrm{N} 3-\mathrm{C} 8-\mathrm{C} 9-\mathrm{C} 10$ & $0.2(2)$ \\
\hline $\mathrm{O} 1-\mathrm{S} 1-\mathrm{C} 4-\mathrm{C} 5$ & $62.68(13)$ & $\mathrm{C} 7-\mathrm{N} 4-\mathrm{C} 10-\mathrm{C} 9$ & $-0.8(2)$ \\
\hline $\mathrm{N} 2-\mathrm{S} 1-\mathrm{C} 4-\mathrm{C} 5$ & $-48.09(13)$ & $\mathrm{C} 8-\mathrm{C} 9-\mathrm{C} 10-\mathrm{N} 4$ & $0.3(2)$ \\
\hline $\mathrm{C} 3-\mathrm{C} 4-\mathrm{C} 5-\mathrm{C} 6$ & $-0.9(2)$ & & \\
\hline
\end{tabular}


Hydrogen-bond geometry $\left(\AA,{ }^{\circ}\right)$

\begin{tabular}{lllll}
\hline$D-\mathrm{H} \cdots A$ & $D-\mathrm{H}$ & $\mathrm{H} \cdots A$ & $D \cdots A$ & $D-\mathrm{H} \cdots A$ \\
\hline $\mathrm{N} 1-\mathrm{H} 1 N \cdots \mathrm{Cl} 1^{\mathrm{i}}$ & $0.91(2)$ & $2.27(2)$ & $3.1484(13)$ & $161.8(19)$ \\
$\mathrm{N} 1-\mathrm{H} 2 N \cdots \mathrm{Cl} 1^{\mathrm{ii}}$ & $0.91(2)$ & $2.27(2)$ & $3.1795(13)$ & $176.0(18)$ \\
$\mathrm{N} 1-\mathrm{H} 3 N \cdots \mathrm{O} 1^{\text {iii }}$ & $0.91(2)$ & $1.99(2)$ & $2.8243(16)$ & $150.2(19)$ \\
$\mathrm{N} 3-\mathrm{H} 4 N \cdots \mathrm{N} 2^{\text {iv }}$ & $0.90(2)$ & $1.98(2)$ & $2.8763(17)$ & $175.9(19)$ \\
$\mathrm{N} 3-\mathrm{H} 4 N \cdots \mathrm{O} 1^{\text {iv }}$ & $0.90(2)$ & $2.54(2)$ & $3.0962(15)$ & $121.1(16)$ \\
$\mathrm{O} 1 W-\mathrm{H} 1 W \cdots \mathrm{Cl} 1$ & $0.88(1)$ & $2.62(1)$ & $3.4607(17)$ & $160(3)$ \\
$\mathrm{O} 1 W-\mathrm{H} 2 W \cdots \mathrm{O} 1 W^{\nu}$ & $0.88(1)$ & $2.18(2)$ & $2.995(4)$ & $153(4)$ \\
$\mathrm{O} 1 W-\mathrm{H} 3 W \cdots \mathrm{O} 1 W^{i}$ & $0.88(1)$ & $2.21(2)$ & $3.030(4)$ & $155(5)$ \\
\hline
\end{tabular}

Symmetry codes: (i) $-x+2,-y+2,-z+1$; (ii) $-x+1,-y+2,-z+1$; (iii) $-x,-y+1,-z$; (iv) $-x,-y+1,-z+1$; (v) $-x+3,-y+2,-z+1$.

\section{(II) 2-\{[(4-azaniumylphenyl)sulfonyl]azanidyl\}pyrimidin-1-ium chloride methanol hemisolvate}

Crystal data

$\mathrm{C}_{10} \mathrm{H}_{11} \mathrm{~N}_{4} \mathrm{O}_{2} \mathrm{~S}^{+} \cdot \mathrm{Cl}^{-} \cdot 0.5 \mathrm{CH}_{4} \mathrm{O}$

$M_{r}=302.76$

Triclinic, $P \overline{1}$

Hall symbol: -P 1

$a=5.6468(2) \AA$

$b=11.2749(4) \AA$

$c=11.8363(8) \AA$

$\alpha=64.254(5)^{\circ}$

$\beta=79.361(6)^{\circ}$

$\gamma=77.687(6)^{\circ}$

$V=659.53(6) \AA^{3}$

\section{Data collection}

Rigaku Saturn724+ (2x2 bin mode) diffractometer

Radiation source: Rotating Anode

Confocal monochromator

profile data from $\omega$-scans

Absorption correction: multi-scan

(CrystalClear-SM Expert; Rigaku, 2013)

$T_{\min }=0.819, T_{\max }=1.000$

\section{Refinement}

Refinement on $F^{2}$

Least-squares matrix: full

$R\left[F^{2}>2 \sigma\left(F^{2}\right)\right]=0.031$

$w R\left(F^{2}\right)=0.082$

$S=1.04$

3028 reflections

201 parameters

1 restraint

Primary atom site location: structure-invariant direct methods
$Z=2$

$F(000)=314$

$D_{\mathrm{x}}=1.525 \mathrm{Mg} \mathrm{m}^{-3}$

Mo $K \alpha$ radiation, $\lambda=0.71073 \AA$

Cell parameters from 10881 reflections

$\theta=3.5-27.5^{\circ}$

$\mu=0.45 \mathrm{~mm}^{-1}$

$T=100 \mathrm{~K}$

Block, light yellow

$0.09 \times 0.06 \times 0.05 \mathrm{~mm}$

11731 measured reflections

3028 independent reflections

2804 reflections with $I>2 \sigma(I)$

$R_{\text {int }}=0.029$

$\theta_{\max }=27.5^{\circ}, \theta_{\min }=3.3^{\circ}$

$h=-7 \rightarrow 7$

$k=-14 \rightarrow 14$

$l=-15 \rightarrow 14$

Secondary atom site location: difference Fourier map

Hydrogen site location: inferred from neighbouring sites

$\mathrm{H}$ atoms treated by a mixture of independent and constrained refinement

$w=1 /\left[\sigma^{2}\left(F_{\mathrm{o}}^{2}\right)+(0.0374 P)^{2}+0.5228 P\right]$

where $P=\left(F_{\mathrm{o}}^{2}+2 F_{\mathrm{c}}^{2}\right) / 3$

$(\Delta / \sigma)_{\max }=0.001$

$\Delta \rho_{\max }=0.40 \mathrm{e} \AA^{-3}$

$\Delta \rho_{\min }=-0.43$ e $\AA^{-3}$ 


\section{Special details}

Experimental. Collected by NCS as 2013 ncs0086.

Geometry. All s.u.'s (except the s.u. in the dihedral angle between two 1.s. planes) are estimated using the full covariance matrix. The cell s.u.'s are taken into account individually in the estimation of s.u.'s in distances, angles and torsion angles; correlations between s.u.'s in cell parameters are only used when they are defined by crystal symmetry. An approximate (isotropic) treatment of cell s.u.'s is used for estimating s.u.'s involving l.s. planes.

Refinement. Methanol disordered about i.

Refinement of $F^{2}$ against ALL reflections. The weighted $R$-factor $w R$ and goodness of fit $S$ are based on $F^{2}$, conventional $R$-factors $R$ are based on $F$, with $F$ set to zero for negative $F^{2}$. The threshold expression of $F^{2}>2 \sigma\left(F^{2}\right)$ is used only for calculating $R$-factors(gt) etc. and is not relevant to the choice of reflections for refinement. $R$-factors based on $F^{2}$ are statistically about twice as large as those based on $F$, and $R$ - factors based on ALL data will be even larger.

Fractional atomic coordinates and isotropic or equivalent isotropic displacement parameters $\left(\AA^{2}\right)$

\begin{tabular}{|c|c|c|c|c|c|}
\hline & $x$ & $y$ & $z$ & $U_{\text {iso }} * / U_{\text {eq }}$ & Occ. $(<1)$ \\
\hline S1 & $0.40767(6)$ & $0.72559(3)$ & $0.22516(3)$ & $0.01372(10)$ & \\
\hline $\mathrm{Cl1}$ & $0.75329(6)$ & $0.38116(4)$ & $0.14375(3)$ & $0.01880(10)$ & \\
\hline O1 & 0.65919 (19) & $0.68725(10)$ & $0.18505(10)$ & $0.0176(2)$ & \\
\hline $\mathrm{O} 2$ & $0.2257(2)$ & $0.70618(10)$ & $0.16657(10)$ & $0.0186(2)$ & \\
\hline N1 & $0.3079(2)$ & $1.31394(12)$ & $0.05936(13)$ & $0.0161(3)$ & \\
\hline $\mathrm{N} 2$ & $0.3892(2)$ & $0.64417(12)$ & $0.37570(11)$ & $0.0150(2)$ & \\
\hline N3 & $0.1813(2)$ & $0.55649(12)$ & $0.57023(12)$ & $0.0150(2)$ & \\
\hline N4 & $-0.0209(2)$ & 0.74149 (13) & 0.41155 (12) & $0.0183(3)$ & \\
\hline $\mathrm{C} 7$ & $0.1794(3)$ & $0.65129(14)$ & 0.44874 (13) & 0.0147 (3) & \\
\hline $\mathrm{C} 8$ & $-0.0120(3)$ & $0.54958(15)$ & $0.65718(14)$ & $0.0172(3)$ & \\
\hline H8 & -0.0051 & 0.4833 & 0.7410 & $0.021^{*}$ & \\
\hline $\mathrm{C} 9$ & $-0.2192(3)$ & $0.63983(16)$ & $0.62264(15)$ & 0.0195 (3) & \\
\hline H9 & -0.3603 & 0.6383 & 0.6808 & $0.023^{*}$ & \\
\hline $\mathrm{C} 10$ & -0.2123 & $0.73386(16)$ & 0.49799 (15) & $0.0204(3)$ & \\
\hline $\mathrm{H} 10$ & -0.3544 & 0.7972 & 0.4727 & $0.024^{*}$ & \\
\hline $\mathrm{C} 4$ & $0.3707(3)$ & 0.89860 (14) & $0.18623(13)$ & $0.0143(3)$ & \\
\hline $\mathrm{C} 3$ & $0.5466(3)$ & $0.95072(15)$ & $0.21162(14)$ & 0.0167 (3) & \\
\hline $\mathrm{H} 3$ & 0.6812 & 0.8934 & 0.2547 & $0.020^{*}$ & \\
\hline $\mathrm{C} 2$ & $0.5231(3)$ & $1.08797(15)$ & 0.17307 (14) & $0.0168(3)$ & \\
\hline $\mathrm{H} 2$ & 0.6386 & 1.1253 & 0.1917 & $0.020^{*}$ & \\
\hline $\mathrm{C} 1$ & $0.3284(3)$ & 1.16919 (14) & $0.10703(13)$ & $0.0144(3)$ & \\
\hline C6 & $0.1533(3)$ & $1.11789(14)$ & $0.08048(14)$ & $0.0160(3)$ & \\
\hline H6 & 0.0221 & 1.1755 & 0.0346 & $0.019^{*}$ & \\
\hline $\mathrm{C} 5$ & 0.1735 & $0.98098(15)$ & $0.12211(14)$ & $0.0159(3)$ & \\
\hline H5 & 0.0534 & 0.9436 & 0.1069 & $0.019 *$ & \\
\hline $\mathrm{O} 3$ & $0.7639(6)$ & $0.1624(4)$ & 0.4251 (3) & $0.0507(8)$ & 0.50 \\
\hline $\mathrm{H} 1 \mathrm{H}$ & $0.794(12)$ & $0.216(5)$ & $0.345(2)$ & $0.076^{*}$ & 0.50 \\
\hline C11 & $0.9255(11)$ & $0.0552(5)$ & $0.4402(5)$ & $0.0568(13)$ & 0.50 \\
\hline H11A & 1.0887 & 0.0758 & 0.4339 & $0.085^{*}$ & 0.50 \\
\hline H11B & 0.9192 & 0.0253 & 0.3746 & $0.085^{*}$ & 0.50 \\
\hline $\mathrm{H} 11 \mathrm{C}$ & 0.8889 & -0.0155 & 0.5234 & $0.085^{*}$ & 0.50 \\
\hline $\mathrm{H} 1 \mathrm{~N}$ & $0.428(4)$ & $1.339(2)$ & 0.0828 (19) & $0.024(5)^{*}$ & \\
\hline $\mathrm{H} 2 \mathrm{~N}$ & $0.319(4)$ & $1.351(2)$ & $-0.022(2)$ & $0.033(6)^{*}$ & \\
\hline
\end{tabular}




\section{supporting information}

\begin{tabular}{|c|c|c|c|c|}
\hline $\mathrm{H} 3 \mathrm{~N}$ & $0.156(5)$ & $1.349(2)$ & $0.085(2)$ & $0.038(6)^{*}$ \\
\hline $\mathrm{H} 4 \mathrm{~N}$ & $0.316(4)$ & $0.497(2)$ & $0.5888(19)$ & $0.027(5) *$ \\
\hline
\end{tabular}

Atomic displacement parameters $\left(\AA^{2}\right)$

\begin{tabular}{lllllll}
\hline & $U^{11}$ & $U^{22}$ & $U^{33}$ & $U^{12}$ & $U^{13}$ & $U^{23}$ \\
\hline S1 & $0.01316(17)$ & $0.01214(17)$ & $0.01348(17)$ & $-0.00231(12)$ & $-0.00062(12)$ & $-0.00321(13)$ \\
C11 & $0.01368(17)$ & $0.01857(18)$ & $0.02504(19)$ & $-0.00376(13)$ & $0.00072(13)$ & $-0.01025(15)$ \\
O1 & $0.0154(5)$ & $0.0164(5)$ & $0.0167(5)$ & $-0.0003(4)$ & $0.0013(4)$ & $-0.0050(4)$ \\
O2 & $0.0206(5)$ & $0.0163(5)$ & $0.0185(5)$ & $-0.0053(4)$ & $-0.0052(4)$ & $-0.0044(4)$ \\
N1 & $0.0145(6)$ & $0.0136(6)$ & $0.0184(6)$ & $-0.0026(5)$ & $-0.0008(5)$ & $-0.0050(5)$ \\
N2 & $0.0131(6)$ & $0.0139(6)$ & $0.0142(6)$ & $-0.0025(4)$ & $0.0002(4)$ & $-0.0026(5)$ \\
N3 & $0.0134(6)$ & $0.0140(6)$ & $0.0151(6)$ & $-0.0021(5)$ & $-0.0011(5)$ & $-0.0039(5)$ \\
N4 & $0.0148(6)$ & $0.0181(6)$ & $0.0182(6)$ & $-0.0011(5)$ & $-0.0015(5)$ & $-0.0047(5)$ \\
C7 & $0.0138(6)$ & $0.0135(6)$ & $0.0165(7)$ & $-0.0034(5)$ & $-0.0017(5)$ & $-0.0054(5)$ \\
C8 & $0.0167(7)$ & $0.0175(7)$ & $0.0167(7)$ & $-0.0060(5)$ & $0.0011(5)$ & $-0.0061(6)$ \\
C9 & $0.0139(7)$ & $0.0234(7)$ & $0.0203(7)$ & $-0.0038(6)$ & $0.0017(5)$ & $-0.0090(6)$ \\
C10 & $0.0141(7)$ & $0.0216(7)$ & $0.0227(8)$ & $0.0001(6)$ & $-0.0022(6)$ & $-0.0077(6)$ \\
C4 & $0.0133(6)$ & $0.0127(6)$ & $0.0141(6)$ & $-0.0026(5)$ & $0.0007(5)$ & $-0.0035(5)$ \\
C3 & $0.0137(6)$ & $0.0166(7)$ & $0.0175(7)$ & $-0.0019(5)$ & $-0.0035(5)$ & $-0.0043(5)$ \\
C2 & $0.0141(6)$ & $0.0172(7)$ & $0.0187(7)$ & $-0.0046(5)$ & $-0.0024(5)$ & $-0.0058(6)$ \\
C1 & $0.0141(6)$ & $0.0125(6)$ & $0.0143(6)$ & $-0.0025(5)$ & $0.0015(5)$ & $-0.0042(5)$ \\
C6 & $0.0129(6)$ & $0.0158(7)$ & $0.0172(7)$ & $-0.0010(5)$ & $-0.0026(5)$ & $-0.0050(5)$ \\
C5 & $0.0130(6)$ & $0.0166(7)$ & $0.0168(7)$ & $-0.0033(5)$ & $-0.0016(5)$ & $-0.0053(5)$ \\
O3 & $0.0399(17)$ & $0.062(2)$ & $0.0376(17)$ & $-0.0020(15)$ & $-0.0035(14)$ & $-0.0118(16)$ \\
C11 & $0.073(4)$ & $0.034(2)$ & $0.064(3)$ & $-0.013(2)$ & $-0.003(3)$ & $-0.020(2)$ \\
& & & & & &
\end{tabular}

Geometric parameters $\left(\AA,{ }^{\circ}\right)$

\begin{tabular}{llll}
\hline $\mathrm{S} 1-\mathrm{O} 2$ & $1.4407(11)$ & $\mathrm{C} 9-\mathrm{H} 9$ & 0.9500 \\
$\mathrm{~S} 1-\mathrm{O} 1$ & $1.4481(11)$ & $\mathrm{C} 10-\mathrm{H} 10$ & 0.9500 \\
$\mathrm{~S} 1-\mathrm{N} 2$ & $1.6061(12)$ & $\mathrm{C} 4-\mathrm{C} 3$ & $1.391(2)$ \\
$\mathrm{S} 1-\mathrm{C} 4$ & $1.7725(14)$ & $\mathrm{C} 4-\mathrm{C} 5$ & $1.392(2)$ \\
$\mathrm{N} 1-\mathrm{C} 1$ & $1.4625(18)$ & $\mathrm{C} 3-\mathrm{C} 2$ & $1.394(2)$ \\
$\mathrm{N} 1-\mathrm{H} 1 \mathrm{~N}$ & $0.91(2)$ & $\mathrm{C} 3-\mathrm{H} 3$ & 0.9500 \\
$\mathrm{~N} 1-\mathrm{H} 2 \mathrm{~N}$ & $0.86(2)$ & $\mathrm{C} 2-\mathrm{C} 1$ & $1.387(2)$ \\
$\mathrm{N} 1-\mathrm{H} 3 \mathrm{~N}$ & $0.92(3)$ & $\mathrm{C} 2-\mathrm{H} 2$ & 0.9500 \\
$\mathrm{~N} 2-\mathrm{C} 7$ & $1.3413(19)$ & $\mathrm{C} 1-\mathrm{C} 6$ & $1.388(2)$ \\
$\mathrm{N} 3-\mathrm{C} 8$ & $1.3449(19)$ & $\mathrm{C} 6-\mathrm{C} 5$ & $1.387(2)$ \\
$\mathrm{N} 3-\mathrm{C} 7$ & $1.3697(19)$ & $\mathrm{C} 6-\mathrm{H} 6$ & 0.9500 \\
$\mathrm{~N} 3-\mathrm{H} 4 \mathrm{~N}$ & $0.89(2)$ & $\mathrm{C} 5-\mathrm{H} 5$ & 0.9500 \\
$\mathrm{~N} 4-\mathrm{C} 10$ & $1.333(2)$ & $\mathrm{O} 3-\mathrm{C} 11$ & $1.314(6)$ \\
$\mathrm{N} 4-\mathrm{C} 7$ & $1.3461(19)$ & $\mathrm{O} 3-\mathrm{H} 1 \mathrm{H}$ & $0.886(10)$ \\
$\mathrm{C} 8-\mathrm{C} 9$ & $1.371(2)$ & $\mathrm{C} 11-\mathrm{H} 11 \mathrm{~A}$ & 0.9800 \\
$\mathrm{C} 8-\mathrm{H} 8$ & 0.9500 & $\mathrm{C} 11-\mathrm{H} 11 \mathrm{~B}$ & 0.9800 \\
$\mathrm{C} 9-\mathrm{C} 10$ & $1.393(2)$ & $\mathrm{C} 11-\mathrm{H} 11 \mathrm{C}$ & 0.9800 \\
& & & 121.7 \\
$\mathrm{O} 2-\mathrm{S} 1-\mathrm{O} 1$ & $116.27(7)$ & $\mathrm{C} 8-\mathrm{C} 9-\mathrm{H} 9$ &
\end{tabular}




\begin{tabular}{|c|c|c|c|}
\hline $\mathrm{O} 2-\mathrm{S} 1-\mathrm{N} 2$ & $113.94(7)$ & $\mathrm{C} 10-\mathrm{C} 9-\mathrm{H} 9$ & 121.7 \\
\hline $\mathrm{O} 1-\mathrm{S} 1-\mathrm{N} 2$ & $103.29(6)$ & $\mathrm{N} 4-\mathrm{C} 10-\mathrm{C} 9$ & $124.82(14)$ \\
\hline $\mathrm{O} 2-\mathrm{S} 1-\mathrm{C} 4$ & $106.85(7)$ & $\mathrm{N} 4-\mathrm{C} 10-\mathrm{H} 10$ & 117.6 \\
\hline $\mathrm{O} 1-\mathrm{S} 1-\mathrm{C} 4$ & $106.51(7)$ & $\mathrm{C} 9-\mathrm{C} 10-\mathrm{H} 10$ & 117.6 \\
\hline $\mathrm{N} 2-\mathrm{S} 1-\mathrm{C} 4$ & $109.69(7)$ & $\mathrm{C} 3-\mathrm{C} 4-\mathrm{C} 5$ & $121.26(13)$ \\
\hline $\mathrm{C} 1-\mathrm{N} 1-\mathrm{H} 1 \mathrm{~N}$ & $112.2(13)$ & $\mathrm{C} 3-\mathrm{C} 4-\mathrm{S} 1$ & $119.87(11)$ \\
\hline $\mathrm{C} 1-\mathrm{N} 1-\mathrm{H} 2 \mathrm{~N}$ & $111.2(15)$ & $\mathrm{C} 5-\mathrm{C} 4-\mathrm{S} 1$ & $118.70(11)$ \\
\hline $\mathrm{H} 1 \mathrm{~N}-\mathrm{N} 1-\mathrm{H} 2 \mathrm{~N}$ & $106.9(19)$ & $\mathrm{C} 4-\mathrm{C} 3-\mathrm{C} 2$ & $119.21(13)$ \\
\hline $\mathrm{C} 1-\mathrm{N} 1-\mathrm{H} 3 \mathrm{~N}$ & $109.9(15)$ & $\mathrm{C} 4-\mathrm{C} 3-\mathrm{H} 3$ & 120.4 \\
\hline $\mathrm{H} 1 \mathrm{~N}-\mathrm{N} 1-\mathrm{H} 3 \mathrm{~N}$ & $111.3(19)$ & $\mathrm{C} 2-\mathrm{C} 3-\mathrm{H} 3$ & 120.4 \\
\hline $\mathrm{H} 2 \mathrm{~N}-\mathrm{N} 1-\mathrm{H} 3 \mathrm{~N}$ & $105(2)$ & $\mathrm{C} 1-\mathrm{C} 2-\mathrm{C} 3$ & $118.95(13)$ \\
\hline $\mathrm{C} 7-\mathrm{N} 2-\mathrm{S} 1$ & $121.68(10)$ & $\mathrm{C} 1-\mathrm{C} 2-\mathrm{H} 2$ & 120.5 \\
\hline $\mathrm{C} 8-\mathrm{N} 3-\mathrm{C} 7$ & $122.36(13)$ & $\mathrm{C} 3-\mathrm{C} 2-\mathrm{H} 2$ & 120.5 \\
\hline $\mathrm{C} 8-\mathrm{N} 3-\mathrm{H} 4 \mathrm{~N}$ & $120.9(14)$ & $\mathrm{C} 2-\mathrm{C} 1-\mathrm{C} 6$ & $122.10(13)$ \\
\hline $\mathrm{C} 7-\mathrm{N} 3-\mathrm{H} 4 \mathrm{~N}$ & $116.7(14)$ & $\mathrm{C} 2-\mathrm{C} 1-\mathrm{N} 1$ & $119.86(13)$ \\
\hline $\mathrm{C} 10-\mathrm{N} 4-\mathrm{C} 7$ & $116.95(13)$ & $\mathrm{C} 6-\mathrm{C} 1-\mathrm{N} 1$ & $118.01(13)$ \\
\hline $\mathrm{N} 2-\mathrm{C} 7-\mathrm{N} 4$ & $125.45(13)$ & $\mathrm{C} 5-\mathrm{C} 6-\mathrm{C} 1$ & $118.78(13)$ \\
\hline $\mathrm{N} 2-\mathrm{C} 7-\mathrm{N} 3$ & $114.12(13)$ & $\mathrm{C} 5-\mathrm{C} 6-\mathrm{H} 6$ & 120.6 \\
\hline $\mathrm{N} 4-\mathrm{C} 7-\mathrm{N} 3$ & $120.43(13)$ & $\mathrm{C} 1-\mathrm{C} 6-\mathrm{H} 6$ & 120.6 \\
\hline $\mathrm{N} 3-\mathrm{C} 8-\mathrm{C} 9$ & $118.82(14)$ & $\mathrm{C} 6-\mathrm{C} 5-\mathrm{C} 4$ & $119.67(14)$ \\
\hline $\mathrm{N} 3-\mathrm{C} 8-\mathrm{H} 8$ & 120.6 & $\mathrm{C} 6-\mathrm{C} 5-\mathrm{H} 5$ & 120.2 \\
\hline $\mathrm{C} 9-\mathrm{C} 8-\mathrm{H} 8$ & 120.6 & $\mathrm{C} 4-\mathrm{C} 5-\mathrm{H} 5$ & 120.2 \\
\hline $\mathrm{C} 8-\mathrm{C} 9-\mathrm{C} 10$ & $116.63(14)$ & $\mathrm{C} 11-\mathrm{O} 3-\mathrm{H} 1 \mathrm{H}$ & $104(4)$ \\
\hline $\mathrm{O} 2-\mathrm{S} 1-\mathrm{N} 2-\mathrm{C} 7$ & $-51.72(14)$ & $\mathrm{N} 2-\mathrm{S} 1-\mathrm{C} 4-\mathrm{C} 3$ & $66.90(13)$ \\
\hline $\mathrm{O} 1-\mathrm{S} 1-\mathrm{N} 2-\mathrm{C} 7$ & $-178.76(12)$ & $\mathrm{O} 2-\mathrm{S} 1-\mathrm{C} 4-\mathrm{C} 5$ & $6.20(13)$ \\
\hline $\mathrm{C} 4-\mathrm{S} 1-\mathrm{N} 2-\mathrm{C} 7$ & $68.00(13)$ & $\mathrm{O} 1-\mathrm{S} 1-\mathrm{C} 4-\mathrm{C} 5$ & $131.09(12)$ \\
\hline $\mathrm{S} 1-\mathrm{N} 2-\mathrm{C} 7-\mathrm{N} 4$ & $-12.9(2)$ & $\mathrm{N} 2-\mathrm{S} 1-\mathrm{C} 4-\mathrm{C} 5$ & $-117.77(12)$ \\
\hline $\mathrm{S} 1-\mathrm{N} 2-\mathrm{C} 7-\mathrm{N} 3$ & $167.34(10)$ & $\mathrm{C} 5-\mathrm{C} 4-\mathrm{C} 3-\mathrm{C} 2$ & $0.6(2)$ \\
\hline $\mathrm{C} 10-\mathrm{N} 4-\mathrm{C} 7-\mathrm{N} 2$ & $-179.34(14)$ & $\mathrm{S} 1-\mathrm{C} 4-\mathrm{C} 3-\mathrm{C} 2$ & $175.85(11)$ \\
\hline $\mathrm{C} 10-\mathrm{N} 4-\mathrm{C} 7-\mathrm{N} 3$ & $0.4(2)$ & $\mathrm{C} 4-\mathrm{C} 3-\mathrm{C} 2-\mathrm{C} 1$ & $-1.9(2)$ \\
\hline $\mathrm{C} 8-\mathrm{N} 3-\mathrm{C} 7-\mathrm{N} 2$ & $179.11(13)$ & $\mathrm{C} 3-\mathrm{C} 2-\mathrm{C} 1-\mathrm{C} 6$ & $1.4(2)$ \\
\hline $\mathrm{C} 8-\mathrm{N} 3-\mathrm{C} 7-\mathrm{N} 4$ & $-0.6(2)$ & $\mathrm{C} 3-\mathrm{C} 2-\mathrm{C} 1-\mathrm{N} 1$ & $-176.48(13)$ \\
\hline $\mathrm{C} 7-\mathrm{N} 3-\mathrm{C} 8-\mathrm{C} 9$ & $0.6(2)$ & $\mathrm{C} 2-\mathrm{C} 1-\mathrm{C} 6-\mathrm{C} 5$ & $0.4(2)$ \\
\hline $\mathrm{N} 3-\mathrm{C} 8-\mathrm{C} 9-\mathrm{C} 10$ & $-0.4(2)$ & $\mathrm{N} 1-\mathrm{C} 1-\mathrm{C} 6-\mathrm{C} 5$ & $178.33(13)$ \\
\hline $\mathrm{C} 7-\mathrm{N} 4-\mathrm{C} 10-\mathrm{C} 9$ & $-0.2(2)$ & $\mathrm{C} 1-\mathrm{C} 6-\mathrm{C} 5-\mathrm{C} 4$ & $-1.7(2)$ \\
\hline $\mathrm{C} 8-\mathrm{C} 9-\mathrm{C} 10-\mathrm{N} 4$ & $0.2(2)$ & $\mathrm{C} 3-\mathrm{C} 4-\mathrm{C} 5-\mathrm{C} 6$ & $1.2(2)$ \\
\hline $\mathrm{O} 2-\mathrm{S} 1-\mathrm{C} 4-\mathrm{C} 3$ & $-169.13(11)$ & $\mathrm{S} 1-\mathrm{C} 4-\mathrm{C} 5-\mathrm{C} 6$ & $-174.09(11)$ \\
\hline $\mathrm{O} 1-\mathrm{S} 1-\mathrm{C} 4-\mathrm{C} 3$ & $-44.24(13)$ & & \\
\hline
\end{tabular}

Hydrogen-bond geometry $\left(\AA,{ }^{\circ}\right)$

\begin{tabular}{lllll}
\hline$D-\mathrm{H} \cdots A$ & $D-\mathrm{H}$ & $\mathrm{H} \cdots A$ & $D \cdots A$ & $D-\mathrm{H} \cdots A$ \\
\hline $\mathrm{N} 1-\mathrm{H} 1 N^{\cdots} \mathrm{C}{ }^{\mathrm{i}} 1^{\mathrm{i}}$ & $0.91(2)$ & $2.30(2)$ & $3.2088(14)$ & $174.1(18)$ \\
$\mathrm{N} 1-\mathrm{H} 2 N \cdots \mathrm{C} 11^{\mathrm{ii}}$ & $0.86(2)$ & $2.70(2)$ & $3.2151(13)$ & $119.8(18)$ \\
$\mathrm{N} 1-\mathrm{H} 2 N \cdots{ }^{i i}$ & $0.86(2)$ & $2.13(2)$ & $2.8701(18)$ & $144(2)$ \\
$\mathrm{N} 1-\mathrm{H} 3 N \cdots \mathrm{C} 1^{\text {iii }}$ & $0.92(3)$ & $2.25(3)$ & $3.1417(14)$ & $163(2)$ \\
$\mathrm{N} 3-\mathrm{H} 4 N \cdots \mathrm{N} 2^{\text {iv }}$ & $0.89(2)$ & $2.01(2)$ & $2.8957(18)$ & $175.4(19)$
\end{tabular}




\begin{tabular}{lllll}
$\mathrm{N} 3-\mathrm{H} 4 N \cdots \mathrm{O} 1^{\text {iv }}$ & $0.89(2)$ & $2.59(2)$ & $3.1218(16)$ & $119.6(16)$ \\
$\mathrm{O} 3-\mathrm{H} 1 H \cdots \mathrm{Cl1}$ & $0.89(1)$ & $2.31(2)$ & $3.170(3)$ & $163(6)$ \\
\hline
\end{tabular}

Symmetry codes: (i) $x, y+1, z$; (ii) $-x+1,-y+2,-z$; (iii) $x-1, y+1, z$; (iv) $-x+1,-y+1,-z+1$.

(III) 2-\{[(4-Azaniumylphenyl)sulfonyl]azanidyl\}pyrimidin-1-ium bromide monohydrate

Crystal data

$\mathrm{C}_{10} \mathrm{H}_{11} \mathrm{~N}_{4} \mathrm{O}_{2} \mathrm{~S}^{+} \cdot \mathrm{Br}^{-} \cdot \mathrm{H}_{2} \mathrm{O}$

$M_{r}=349.21$

Monoclinic, $P 2_{1} / n$

Hall symbol: -P 2 yn

$a=11.9420(3) \AA$

$b=5.6295(2) \AA$

$c=21.0659(6) \AA$

$\beta=98.380(3)^{\circ}$

$V=1401.09(7) \AA^{3}$

$Z=4$

\section{Data collection}

Oxford Diffraction Xcalibur E diffractometer

Radiation source: fine-focus sealed tube

Graphite monochromator

$\omega$ scans

Absorption correction: multi-scan

(CrysAlis PRO; Oxford Diffraction, 2010)

$T_{\min }=0.616, T_{\max }=1.000$

Refinement

Refinement on $F^{2}$

Least-squares matrix: full

$R\left[F^{2}>2 \sigma\left(F^{2}\right)\right]=0.029$

$w R\left(F^{2}\right)=0.072$

$S=1.06$

3353 reflections

203 parameters

5 restraints

Primary atom site location: structure-invariant direct methods
$F(000)=704$

$D_{\mathrm{x}}=1.656 \mathrm{Mg} \mathrm{m}^{-3}$

Mo $K \alpha$ radiation, $\lambda=0.71073 \AA$

Cell parameters from 4492 reflections

$\theta=3.2-29.8^{\circ}$

$\mu=3.09 \mathrm{~mm}^{-1}$

$T=123 \mathrm{~K}$

Plate, colourless

$0.30 \times 0.22 \times 0.04 \mathrm{~mm}$

8186 measured reflections

3353 independent reflections

2868 reflections with $I>2 \sigma(I)$

$R_{\text {int }}=0.024$

$\theta_{\max }=28.0^{\circ}, \theta_{\min }=3.7^{\circ}$

$h=-15 \rightarrow 15$

$k=-6 \rightarrow 7$

$l=-25 \rightarrow 27$

Secondary atom site location: difference Fourier map

Hydrogen site location: inferred from neighbouring sites

$\mathrm{H}$ atoms treated by a mixture of independent and constrained refinement

$w=1 /\left[\sigma^{2}\left(F_{\mathrm{o}}^{2}\right)+(0.0308 P)^{2}+0.9997 P\right]$ where $P=\left(F_{\mathrm{o}}^{2}+2 F_{\mathrm{c}}^{2}\right) / 3$

$(\Delta / \sigma)_{\max }=0.001$

$\Delta \rho_{\max }=0.46$ e $\AA^{-3}$

$\Delta \rho_{\min }=-0.52$ e $\AA^{-3}$

\section{Special details}

Experimental. Absorption correction: CrysAlisPro, Oxford Diffraction Ltd., Version 1.171.34.40 (release 27-08-2010 CrysAlis171 .NET) (compiled Aug 27 2010,11:50:40) Empirical absorption correction using spherical harmonics, implemented in SCALE3 ABSPACK scaling algorithm.

Geometry. All s.u.'s (except the s.u. in the dihedral angle between two 1.s. planes) are estimated using the full covariance matrix. The cell s.u.'s are taken into account individually in the estimation of s.u.'s in distances, angles and torsion angles; correlations between s.u.'s in cell parameters are only used when they are defined by crystal symmetry. An approximate (isotropic) treatment of cell s.u.'s is used for estimating s.u.'s involving l.s. planes.

Refinement. Refinement of $F^{2}$ against ALL reflections. The weighted $R$-factor $w R$ and goodness of fit $S$ are based on $F^{2}$, conventional $R$-factors $R$ are based on $F$, with $F$ set to zero for negative $F^{2}$. The threshold expression of $F^{2}>2 \sigma\left(F^{2}\right)$ is used only for calculating $R$-factors (gt) etc. and is not relevant to the choice of reflections for refinement. $R$-factors based on $F^{2}$ are statistically about twice as large as those based on $F$, and $R$ - factors based on ALL data will be even larger. 


\section{supporting information}

Fractional atomic coordinates and isotropic or equivalent isotropic displacement parameters $\left(\AA^{2}\right)$

\begin{tabular}{|c|c|c|c|c|c|}
\hline & $x$ & $y$ & $z$ & $U_{\text {iso }} * / U_{\text {eq }}$ & Occ. $(<1)$ \\
\hline $\mathrm{Br} 1$ & $0.401273(18)$ & $0.49939(4)$ & $0.747817(10)$ & $0.01647(8)$ & \\
\hline S1 & $0.76326(4)$ & $0.82481(10)$ & $1.03211(2)$ & $0.01199(12)$ & \\
\hline $\mathrm{O} 1$ & $0.74525(12)$ & $0.5931(3)$ & $1.05768(8)$ & $0.0183(3)$ & \\
\hline $\mathrm{O} 2$ & $0.76800(13)$ & $1.0205(3)$ & $1.07703(8)$ & $0.0162(3)$ & \\
\hline N1 & $0.37146(16)$ & $0.9984(4)$ & $0.82655(9)$ & $0.0137(4)$ & \\
\hline $\mathrm{H} 1 \mathrm{~N}$ & $0.360(2)$ & $0.870(5)$ & $0.8004(13)$ & $0.021 *$ & \\
\hline $\mathrm{H} 2 \mathrm{~N}$ & $0.310(3)$ & $1.006(4)$ & $0.8447(14)$ & $0.021 *$ & \\
\hline $\mathrm{H} 3 \mathrm{~N}$ & $0.378(2)$ & $1.127(5)$ & $0.8058(13)$ & $0.021^{*}$ & \\
\hline $\mathrm{N} 2$ & $0.87783(14)$ & $0.8530(3)$ & $1.00155(9)$ & $0.0131(4)$ & \\
\hline N3 & $0.99972(14)$ & $0.7555(3)$ & $0.93244(9)$ & $0.0129(4)$ & \\
\hline $\mathrm{H} 4 \mathrm{~N}$ & $1.0374(18)$ & $0.871(3)$ & $0.9527(11)$ & $0.015^{*}$ & \\
\hline N4 & $0.83362(16)$ & $0.5297(3)$ & $0.92931(10)$ & $0.0181(4)$ & \\
\hline $\mathrm{C} 4$ & $0.64922(16)$ & $0.8809(4)$ & $0.97023(10)$ & $0.0118(4)$ & \\
\hline $\mathrm{C} 3$ & $0.56750(17)$ & 0.7068 & $0.95621(10)$ & $0.0131(4)$ & \\
\hline $\mathrm{H} 3$ & 0.5746 & 0.5596 & 0.9785 & $0.016^{*}$ & \\
\hline $\mathrm{C} 2$ & $0.47470(17)$ & $0.7483(4)$ & $0.90921(10)$ & $0.0138(4)$ & \\
\hline $\mathrm{H} 2$ & 0.4164 & 0.6332 & 0.9002 & $0.017^{*}$ & \\
\hline $\mathrm{C} 1$ & $0.46949(17)$ & $0.9603(4)$ & $0.87620(10)$ & $0.0116(4)$ & \\
\hline C6 & $0.55169(17)$ & $1.1346(4)$ & $0.88907(10)$ & $0.0146(4)$ & \\
\hline H6 & 0.5464 & 1.2783 & 0.8652 & $0.017^{*}$ & \\
\hline $\mathrm{C} 5$ & $0.64198(18)$ & $1.0956(4)$ & $0.93754(11)$ & $0.0153(4)$ & \\
\hline H5 & 0.6980 & 1.2144 & 0.9482 & $0.018^{*}$ & \\
\hline $\mathrm{C} 7$ & $0.90044(17)$ & $0.7083(4)$ & $0.95472(10)$ & $0.0129(4)$ & \\
\hline $\mathrm{C} 10$ & $0.86942(19)$ & $0.4047(5)$ & $0.88206(12)$ & $0.0217(5)$ & \\
\hline H10 & 0.8229 & 0.2780 & 0.8638 & $0.026^{*}$ & \\
\hline $\mathrm{C} 9$ & $0.9693(2)$ & $0.4467(4)$ & $0.85792(12)$ & $0.0201(5)$ & \\
\hline H9 & 0.9912 & 0.3532 & 0.8243 & $0.024 *$ & \\
\hline $\mathrm{C} 8$ & $1.03513(18)$ & $0.6303(4)$ & $0.88507(10)$ & $0.0156(5)$ & \\
\hline H8 & 1.1047 & 0.6680 & 0.8705 & $0.019^{*}$ & \\
\hline O1W & $0.8026(5)$ & $0.0239(18)$ & $0.7626(4)$ & $0.0386(16)$ & $0.453(10)$ \\
\hline H1W & $0.826(8)$ & $-0.122(6)$ & $0.759(5)$ & $0.058^{*}$ & 0.45 \\
\hline $\mathrm{H} 2 \mathrm{~W}$ & $0.830(9)$ & $0.088(16)$ & $0.730(4)$ & $0.058^{*}$ & 0.45 \\
\hline $\mathrm{O} 2 \mathrm{~W}$ & $0.8094(12)$ & $-0.082(3)$ & $0.7675(8)$ & $0.0386(16)$ & $0.248(10)$ \\
\hline $\mathrm{O} 3 \mathrm{~W}$ & $0.8131(13)$ & $0.141(3)$ & $0.7321(9)$ & $0.0386(16)$ & $0.161(6)$ \\
\hline O4W & $0.7780(11)$ & $0.300(3)$ & $0.7019(8)$ & $0.0386(16)$ & $0.139(4)$ \\
\hline
\end{tabular}

Atomic displacement parameters $\left(\AA^{2}\right)$

\begin{tabular}{lllllll}
\hline & $U^{11}$ & $U^{22}$ & $U^{33}$ & $U^{12}$ & $U^{13}$ & $U^{23}$ \\
\hline $\mathrm{Br} 1$ & $0.01972(12)$ & $0.01516(13)$ & $0.01408(12)$ & $-0.00096(8)$ & $0.00096(8)$ & $0.00103(8)$ \\
$\mathrm{S} 1$ & $0.0089(2)$ & $0.0151(3)$ & $0.0120(2)$ & $-0.0023(2)$ & $0.00132(17)$ & $0.0010(2)$ \\
$\mathrm{O} 1$ & $0.0135(7)$ & $0.0192(8)$ & $0.0213(9)$ & $-0.0033(6)$ & $-0.0004(6)$ & $0.0081(7)$ \\
$\mathrm{O} 2$ & $0.0128(7)$ & $0.0225(9)$ & $0.0138(8)$ & $-0.0027(6)$ & $0.0033(6)$ & $-0.0046(6)$ \\
$\mathrm{N} 1$ & $0.0112(8)$ & $0.0162(10)$ & $0.0131(9)$ & $-0.0013(7)$ & $0.0000(7)$ & $0.0021(8)$ \\
$\mathrm{N} 2$ & $0.0093(8)$ & $0.0150(10)$ & $0.0154(9)$ & $-0.0025(7)$ & $0.0033(6)$ & $-0.0017(7)$
\end{tabular}


supporting information

$\begin{array}{lllllll}\mathrm{N} 3 & 0.0105(8) & 0.0136(9) & 0.0145(9) & -0.0018(7) & 0.0017(6) & -0.0008(7) \\ \mathrm{N} 4 & 0.0156(9) & 0.0180(11) & 0.0208(10) & -0.0057(7) & 0.0033(7) & -0.0036(8) \\ \mathrm{C} 4 & 0.0098(9) & 0.0141(11) & 0.0117(10) & 0.0000(8) & 0.0021(7) & 0.0001(8) \\ \mathrm{C} 3 & 0.0135(10) & 0.0116(11) & 0.0141(10) & -0.0018(8) & 0.0017(8) & 0.0035(8) \\ \mathrm{C} 2 & 0.0116(9) & 0.0127(11) & 0.0170(11) & -0.0043(8) & 0.0024(8) & -0.0005(8) \\ \mathrm{C} 1 & 0.0093(9) & 0.0162(11) & 0.0094(10) & 0.0005(8) & 0.0014(7) & -0.0004(8) \\ \mathrm{C} 6 & 0.0168(10) & 0.0118(11) & 0.0149(11) & -0.0016(8) & 0.0019(8) & 0.0018(8) \\ \mathrm{C} 5 & 0.0140(10) & 0.0129(11) & 0.0185(11) & -0.0052(8) & 0.0008(8) & 0.0010(9) \\ \text { C7 } & 0.0115(9) & 0.0119(11) & 0.0151(11) & -0.0001(8) & 0.0011(7) & 0.0029(8) \\ \text { C10 } & 0.0209(11) & 0.0205(12) & 0.0235(13) & -0.0062(10) & 0.0027(9) & -0.0061(10) \\ \text { C9 } & 0.0219(11) & 0.0197(13) & 0.0194(12) & 0.0007(9) & 0.0052(9) & -0.0070(9) \\ \text { C8 } & 0.0127(10) & 0.0188(12) & 0.0155(11) & 0.0025(8) & 0.0030(8) & 0.0021(9) \\ \text { O1W } & 0.0258(17) & 0.037(5) & 0.053(3) & -0.003(3) & 0.0057(17) & -0.018(3) \\ \text { O2W } & 0.0258(17) & 0.037(5) & 0.053(3) & -0.003(3) & 0.0057(17) & -0.018(3) \\ \text { O3W } & 0.0258(17) & 0.037(5) & 0.053(3) & -0.003(3) & 0.0057(17) & -0.018(3) \\ \text { O4W } & 0.0258(17) & 0.037(5) & 0.053(3) & -0.003(3) & 0.0057(17) & -0.018(3) \\ & & & & & & \end{array}$

Geometric parameters $\left(\stackrel{A}{ }{ }^{\circ}\right)$

\begin{tabular}{|c|c|c|c|}
\hline $\mathrm{S} 1-\mathrm{O} 1$ & $1.4394(17)$ & $\mathrm{C} 2-\mathrm{C} 1$ & $1.378(3)$ \\
\hline $\mathrm{S} 1-\mathrm{O} 2$ & $1.4480(16)$ & $\mathrm{C} 2-\mathrm{H} 2$ & 0.9500 \\
\hline $\mathrm{S} 1-\mathrm{N} 2$ & $1.6026(18)$ & $\mathrm{C} 1-\mathrm{C} 6$ & $1.387(3)$ \\
\hline $\mathrm{S} 1-\mathrm{C} 4$ & $1.771(2)$ & $\mathrm{C} 6-\mathrm{C} 5$ & $1.390(3)$ \\
\hline $\mathrm{N} 1-\mathrm{C} 1$ & $1.468(3)$ & $\mathrm{C} 6-\mathrm{H} 6$ & 0.9500 \\
\hline $\mathrm{N} 1-\mathrm{H} 1 \mathrm{~N}$ & $0.91(3)$ & $\mathrm{C} 5-\mathrm{H} 5$ & 0.9500 \\
\hline $\mathrm{N} 1-\mathrm{H} 2 \mathrm{~N}$ & $0.88(3)$ & $\mathrm{C} 10-\mathrm{C} 9$ & $1.384(3)$ \\
\hline $\mathrm{N} 1-\mathrm{H} 3 \mathrm{~N}$ & $0.85(3)$ & $\mathrm{C} 10-\mathrm{H} 10$ & 0.9500 \\
\hline $\mathrm{N} 2-\mathrm{C} 7$ & $1.337(3)$ & $\mathrm{C} 9-\mathrm{C} 8$ & $1.372(3)$ \\
\hline $\mathrm{N} 3-\mathrm{C} 8$ & $1.340(3)$ & C9-H9 & 0.9500 \\
\hline N3-C7 & $1.363(3)$ & $\mathrm{C} 8-\mathrm{H} 8$ & 0.9500 \\
\hline $\mathrm{N} 3-\mathrm{H} 4 \mathrm{~N}$ & $0.869(10)$ & $\mathrm{O} 1 \mathrm{~W}-\mathrm{H} 1 \mathrm{~W}$ & $0.876(10)$ \\
\hline $\mathrm{N} 4-\mathrm{C} 10$ & $1.339(3)$ & $\mathrm{O} 1 \mathrm{~W}-\mathrm{H} 2 \mathrm{~W}$ & 0.877 (10) \\
\hline $\mathrm{N} 4-\mathrm{C} 7$ & $1.345(3)$ & $\mathrm{O} 2 \mathrm{~W}-\mathrm{O} 3 \mathrm{~W}$ & $1.46(2)$ \\
\hline $\mathrm{C} 4-\mathrm{C} 3$ & $1.384(3)$ & $\mathrm{O} 2 \mathrm{~W}-\mathrm{O} 4 \mathrm{~W}^{\mathrm{i}}$ & $1.46(2)$ \\
\hline $\mathrm{C} 4-\mathrm{C} 5$ & $1.387(3)$ & $\mathrm{O} 3 \mathrm{~W}-\mathrm{O} 4 \mathrm{~W}$ & $1.14(2)$ \\
\hline $\mathrm{C} 3-\mathrm{C} 2$ & $1.394(3)$ & $\mathrm{O} 4 \mathrm{~W}-\mathrm{O} 2 \mathrm{~W}^{\mathrm{ii}}$ & $1.46(2)$ \\
\hline $\mathrm{C} 3-\mathrm{H} 3$ & 0.9500 & & \\
\hline $\mathrm{O} 1-\mathrm{S} 1-\mathrm{O} 2$ & $116.03(10)$ & $\mathrm{C} 1-\mathrm{C} 2-\mathrm{H} 2$ & 120.8 \\
\hline $\mathrm{O} 1-\mathrm{S} 1-\mathrm{N} 2$ & $115.02(10)$ & $\mathrm{C} 3-\mathrm{C} 2-\mathrm{H} 2$ & 120.8 \\
\hline $\mathrm{O} 2-\mathrm{S} 1-\mathrm{N} 2$ & $103.46(9)$ & $\mathrm{C} 2-\mathrm{C} 1-\mathrm{C} 6$ & 122.39 (19) \\
\hline $\mathrm{O} 1-\mathrm{S} 1-\mathrm{C} 4$ & $107.23(10)$ & $\mathrm{C} 2-\mathrm{C} 1-\mathrm{N} 1$ & $117.33(18)$ \\
\hline $\mathrm{O} 2-\mathrm{S} 1-\mathrm{C} 4$ & $107.34(10)$ & $\mathrm{C} 6-\mathrm{C} 1-\mathrm{N} 1$ & 120.27 (19) \\
\hline $\mathrm{N} 2-\mathrm{S} 1-\mathrm{C} 4$ & $107.26(10)$ & $\mathrm{C} 1-\mathrm{C} 6-\mathrm{C} 5$ & $118.9(2)$ \\
\hline $\mathrm{C} 1-\mathrm{N} 1-\mathrm{H} 1 \mathrm{~N}$ & $110.5(16)$ & $\mathrm{C} 1-\mathrm{C} 6-\mathrm{H} 6$ & 120.6 \\
\hline $\mathrm{C} 1-\mathrm{N} 1-\mathrm{H} 2 \mathrm{~N}$ & $109.2(19)$ & $\mathrm{C} 5-\mathrm{C} 6-\mathrm{H} 6$ & 120.6 \\
\hline $\mathrm{H} 1 \mathrm{~N}-\mathrm{N} 1-\mathrm{H} 2 \mathrm{~N}$ & $104(2)$ & $\mathrm{C} 4-\mathrm{C} 5-\mathrm{C} 6$ & $119.2(2)$ \\
\hline $\mathrm{C} 1-\mathrm{N} 1-\mathrm{H} 3 \mathrm{~N}$ & $111.6(18)$ & $\mathrm{C} 4-\mathrm{C} 5-\mathrm{H} 5$ & 120.4 \\
\hline
\end{tabular}




\begin{tabular}{|c|c|c|c|}
\hline $\mathrm{H} 1 \mathrm{~N}-\mathrm{N} 1-\mathrm{H} 3 \mathrm{~N}$ & $113(3)$ & $\mathrm{C} 6-\mathrm{C} 5-\mathrm{H} 5$ & 120.4 \\
\hline $\mathrm{H} 2 \mathrm{~N}-\mathrm{N} 1-\mathrm{H} 3 \mathrm{~N}$ & $109(2)$ & $\mathrm{N} 2-\mathrm{C} 7-\mathrm{N} 4$ & $125.2(2)$ \\
\hline $\mathrm{C} 7-\mathrm{N} 2-\mathrm{S} 1$ & $120.81(15)$ & $\mathrm{N} 2-\mathrm{C} 7-\mathrm{N} 3$ & $114.51(18)$ \\
\hline $\mathrm{C} 8-\mathrm{N} 3-\mathrm{C} 7$ & $122.61(19)$ & $\mathrm{N} 4-\mathrm{C} 7-\mathrm{N} 3$ & $120.2(2)$ \\
\hline $\mathrm{C} 8-\mathrm{N} 3-\mathrm{H} 4 \mathrm{~N}$ & $124.3(17)$ & $\mathrm{N} 4-\mathrm{C} 10-\mathrm{C} 9$ & $124.7(2)$ \\
\hline $\mathrm{C} 7-\mathrm{N} 3-\mathrm{H} 4 \mathrm{~N}$ & $113.1(17)$ & $\mathrm{N} 4-\mathrm{C} 10-\mathrm{H} 10$ & 117.7 \\
\hline $\mathrm{C} 10-\mathrm{N} 4-\mathrm{C} 7$ & $117.0(2)$ & $\mathrm{C} 9-\mathrm{C} 10-\mathrm{H} 10$ & 117.7 \\
\hline $\mathrm{C} 3-\mathrm{C} 4-\mathrm{C} 5$ & $121.32(18)$ & $\mathrm{C} 8-\mathrm{C} 9-\mathrm{C} 10$ & $116.6(2)$ \\
\hline $\mathrm{C} 3-\mathrm{C} 4-\mathrm{S} 1$ & $118.25(16)$ & $\mathrm{C} 8-\mathrm{C} 9-\mathrm{H} 9$ & 121.7 \\
\hline $\mathrm{C} 5-\mathrm{C} 4-\mathrm{S} 1$ & $120.42(15)$ & $\mathrm{C} 10-\mathrm{C} 9-\mathrm{H} 9$ & 121.7 \\
\hline $\mathrm{C} 4-\mathrm{C} 3-\mathrm{C} 2$ & $119.7(2)$ & $\mathrm{N} 3-\mathrm{C} 8-\mathrm{C} 9$ & $118.9(2)$ \\
\hline $\mathrm{C} 4-\mathrm{C} 3-\mathrm{H} 3$ & 120.2 & $\mathrm{~N} 3-\mathrm{C} 8-\mathrm{H} 8$ & 120.6 \\
\hline $\mathrm{C} 2-\mathrm{C} 3-\mathrm{H} 3$ & 120.2 & $\mathrm{C} 9-\mathrm{C} 8-\mathrm{H} 8$ & 120.6 \\
\hline $\mathrm{C} 1-\mathrm{C} 2-\mathrm{C} 3$ & $118.46(19)$ & $\mathrm{H} 1 \mathrm{~W}-\mathrm{O} 1 \mathrm{~W}-\mathrm{H} 2 \mathrm{~W}$ & $99(2)$ \\
\hline $\mathrm{O} 1-\mathrm{S} 1-\mathrm{N} 2-\mathrm{C} 7$ & $55.99(19)$ & $\mathrm{N} 1-\mathrm{C} 1-\mathrm{C} 6-\mathrm{C} 5$ & $178.3(2)$ \\
\hline $\mathrm{O} 2-\mathrm{S} 1-\mathrm{N} 2-\mathrm{C} 7$ & $-176.45(16)$ & $\mathrm{C} 3-\mathrm{C} 4-\mathrm{C} 5-\mathrm{C} 6$ & $-1.0(3)$ \\
\hline $\mathrm{C} 4-\mathrm{S} 1-\mathrm{N} 2-\mathrm{C} 7$ & $-63.17(19)$ & $\mathrm{S} 1-\mathrm{C} 4-\mathrm{C} 5-\mathrm{C} 6$ & $179.84(18)$ \\
\hline $\mathrm{O} 1-\mathrm{S} 1-\mathrm{C} 4-\mathrm{C} 3$ & $-0.9(2)$ & $\mathrm{C} 1-\mathrm{C} 6-\mathrm{C} 5-\mathrm{C} 4$ & $2.0(3)$ \\
\hline $\mathrm{O} 2-\mathrm{S} 1-\mathrm{C} 4-\mathrm{C} 3$ & $-126.17(18)$ & $\mathrm{S} 1-\mathrm{N} 2-\mathrm{C} 7-\mathrm{N} 4$ & $-0.6(3)$ \\
\hline $\mathrm{N} 2-\mathrm{S} 1-\mathrm{C} 4-\mathrm{C} 3$ & $123.19(18)$ & $\mathrm{S} 1-\mathrm{N} 2-\mathrm{C} 7-\mathrm{N} 3$ & $178.80(14)$ \\
\hline $\mathrm{O} 1-\mathrm{S} 1-\mathrm{C} 4-\mathrm{C} 5$ & $178.30(18)$ & $\mathrm{C} 10-\mathrm{N} 4-\mathrm{C} 7-\mathrm{N} 2$ & $179.2(2)$ \\
\hline $\mathrm{O} 2-\mathrm{S} 1-\mathrm{C} 4-\mathrm{C} 5$ & $53.0(2)$ & $\mathrm{C} 10-\mathrm{N} 4-\mathrm{C} 7-\mathrm{N} 3$ & $-0.1(3)$ \\
\hline $\mathrm{N} 2-\mathrm{S} 1-\mathrm{C} 4-\mathrm{C} 5$ & $-57.6(2)$ & $\mathrm{C} 8-\mathrm{N} 3-\mathrm{C} 7-\mathrm{N} 2$ & $-179.16(19)$ \\
\hline $\mathrm{C} 5-\mathrm{C} 4-\mathrm{C} 3-\mathrm{C} 2$ & $-1.2(3)$ & $\mathrm{C} 8-\mathrm{N} 3-\mathrm{C} 7-\mathrm{N} 4$ & $0.2(3)$ \\
\hline $\mathrm{S} 1-\mathrm{C} 4-\mathrm{C} 3-\mathrm{C} 2$ & $177.92(17)$ & $\mathrm{C} 7-\mathrm{N} 4-\mathrm{C} 10-\mathrm{C} 9$ & $-0.1(4)$ \\
\hline $\mathrm{C} 4-\mathrm{C} 3-\mathrm{C} 2-\mathrm{C} 1$ & $2.4(3)$ & $\mathrm{N} 4-\mathrm{C} 10-\mathrm{C} 9-\mathrm{C} 8$ & $0.1(4)$ \\
\hline $\mathrm{C} 3-\mathrm{C} 2-\mathrm{C} 1-\mathrm{C} 6$ & $-1.5(3)$ & $\mathrm{C} 7-\mathrm{N} 3-\mathrm{C} 8-\mathrm{C} 9$ & $-0.2(3)$ \\
\hline $\mathrm{C} 3-\mathrm{C} 2-\mathrm{C} 1-\mathrm{N} 1$ & $179.4(2)$ & $\mathrm{C} 10-\mathrm{C} 9-\mathrm{C} 8-\mathrm{N} 3$ & $0.1(3)$ \\
\hline $\mathrm{C} 2-\mathrm{C} 1-\mathrm{C} 6-\mathrm{C} 5$ & $-0.8(3)$ & & \\
\hline
\end{tabular}

Symmetry codes: (i) $-x+3 / 2, y-1 / 2,-z+3 / 2$; (ii) $-x+3 / 2, y+1 / 2,-z+3 / 2$.

Hydrogen-bond geometry $\left(A,{ }^{\circ}\right)$

\begin{tabular}{lllll}
\hline$D-\mathrm{H}^{\cdots} A$ & $D-\mathrm{H}$ & $\mathrm{H} \cdots A$ & $D \cdots A$ & $D-\mathrm{H} \cdots A$ \\
\hline $\mathrm{N} 1-\mathrm{H} 1 N \cdots \mathrm{Br} 1$ & $0.91(3)$ & $2.44(3)$ & $3.308(2)$ & $159(2)$ \\
$\mathrm{N} 1-\mathrm{H} 2 N \cdots \mathrm{O} 2^{i i i}$ & $0.88(3)$ & $2.01(3)$ & $2.810(3)$ & $150(3)$ \\
$\mathrm{N} 1-\mathrm{H} 2 N \cdots \mathrm{Br}^{\mathrm{iv}}$ & $0.88(3)$ & $2.95(3)$ & $3.4022(18)$ & $114(2)$ \\
$\mathrm{N} 1-\mathrm{H} 3 N \cdots \mathrm{Br}^{\mathrm{v}}$ & $0.85(3)$ & $2.46(3)$ & $3.317(2)$ & $178(2)$ \\
$\mathrm{N} 3-\mathrm{H} 4 N \cdots \mathrm{N} 2^{\mathrm{vi}}$ & $0.87(1)$ & $2.02(1)$ & $2.887(2)$ & $179(2)$ \\
$\mathrm{N} 3-\mathrm{H} 4 N \cdots \mathrm{O} 2^{\mathrm{vi}}$ & $0.87(1)$ & $2.57(2)$ & $3.080(2)$ & $119(2)$ \\
\hline
\end{tabular}

Symmetry codes: (iii) $-x+1,-y+2,-z+2$; (iv) $-x+1 / 2, y+1 / 2,-z+3 / 2$; (v) $x, y+1, z$; (vi) $-x+2,-y+2,-z+2$. 


\section{supporting information}

\section{(IV) Bis(2-\{[(4-azaniumylphenyl)sulfonyl]azanidyl\}pyrimidin-1-ium) tetraiodide}

Crystal data

$2 \mathrm{C}_{10} \mathrm{H}_{11} \mathrm{~N}_{4} \mathrm{O}_{2} \mathrm{~S}^{+} \cdot \mathrm{I}_{4}^{2-}$

$M_{r}=1010.18$

Triclinic, $P \overline{1}$

Hall symbol: -P 1

$a=5.7792(4) \AA$

$b=12.0002(7) \AA$

$c=12.5214(9) \AA$

$\alpha=61.964(7)^{\circ}$

$\beta=87.745(5)^{\circ}$

$\gamma=76.426(5)^{\circ}$

$V=742.51(9) \AA^{3}$

Data collection

Oxford Diffraction Xcalibur E diffractometer

Radiation source: fine-focus sealed tube

Graphite monochromator

$\omega$ scans

Absorption correction: analytical

[CrysAlis PRO (Oxford Diffraction, 2010), based on expressions derived by Clark \& Reid (1995)]

\section{Refinement}

Refinement on $F^{2}$

Least-squares matrix: full

$R\left[F^{2}>2 \sigma\left(F^{2}\right)\right]=0.032$

$w R\left(F^{2}\right)=0.062$

$S=1.04$

3595 reflections

184 parameters

0 restraints

Primary atom site location: structure-invariant

direct methods
$Z=1$

$F(000)=474$

$D_{\mathrm{x}}=2.259 \mathrm{Mg} \mathrm{m}^{-3}$

Mo $K \alpha$ radiation, $\lambda=0.71073 \AA$

Cell parameters from 3604 reflections

$\theta=3.3-29.4^{\circ}$

$\mu=4.38 \mathrm{~mm}^{-1}$

$T=123 \mathrm{~K}$

Plate, red

$0.25 \times 0.15 \times 0.02 \mathrm{~mm}$

$T_{\min }=0.523, T_{\max }=0.905$

7055 measured reflections

3595 independent reflections

2996 reflections with $I>2 \sigma(I)$

$R_{\text {int }}=0.033$

$\theta_{\max }=29.4^{\circ}, \theta_{\min }=3.3^{\circ}$

$h=-7 \rightarrow 7$

$k=-16 \rightarrow 15$

$l=-16 \rightarrow 16$

Secondary atom site location: difference Fourier map

Hydrogen site location: inferred from neighbouring sites

$\mathrm{H}$ atoms treated by a mixture of independent and constrained refinement

$w=1 /\left[\sigma^{2}\left(F_{\mathrm{o}}^{2}\right)+(0.0159 P)^{2}\right]$

where $P=\left(F_{\mathrm{o}}^{2}+2 F_{\mathrm{c}}^{2}\right) / 3$

$(\Delta / \sigma)_{\max }=0.001$

$\Delta \rho_{\max }=0.66 \mathrm{e} \AA^{-3}$

$\Delta \rho_{\min }=-0.93$ e $\AA^{-3}$

\section{Special details}

Experimental. Absorption correction: CrysAlisPro, Oxford Diffraction Ltd., Version 1.171.34.40 (release 27-08-2010 CrysAlis171 .NET) (compiled Aug 27 2010,11:50:40) Analytical numeric absorption correction using a multifaceted crystal model based on expressions derived by R.C. Clark \& J.S. Reid. (Clark, R. C. \& Reid, J. S. (1995). Acta Cryst. A51, 887-897)

Geometry. All s.u.'s (except the s.u. in the dihedral angle between two 1.s. planes) are estimated using the full covariance matrix. The cell s.u.'s are taken into account individually in the estimation of s.u.'s in distances, angles and torsion angles; correlations between s.u.'s in cell parameters are only used when they are defined by crystal symmetry. An approximate (isotropic) treatment of cell s.u.'s is used for estimating s.u.'s involving l.s. planes.

Refinement. Refinement of $F^{2}$ against ALL reflections. The weighted $R$-factor $w R$ and goodness of fit $S$ are based on $F^{2}$, conventional $R$-factors $R$ are based on $F$, with $F$ set to zero for negative $F^{2}$. The threshold expression of $F^{2}>2 \sigma\left(F^{2}\right)$ is used only for calculating $R$-factors(gt) etc. and is not relevant to the choice of reflections for refinement. $R$-factors based on $F^{2}$ are statistically about twice as large as those based on $F$, and $R$ - factors based on ALL data will be even larger. 
Fractional atomic coordinates and isotropic or equivalent isotropic displacement parameters $\left(\AA^{2}\right)$

\begin{tabular}{|c|c|c|c|c|}
\hline & $x$ & $y$ & $z$ & $U_{\text {iso }} * / U_{\text {eq }}$ \\
\hline I1 & $-0.24113(4)$ & $0.47686(3)$ & $0.64525(2)$ & $0.01501(8)$ \\
\hline I2 & $-0.41230(5)$ & $0.49574(3)$ & $0.89742(3)$ & $0.02602(9)$ \\
\hline S1 & $0.10433(17)$ & $1.04954(10)$ & $0.73009(8)$ & $0.0122(2)$ \\
\hline $\mathrm{O} 1$ & $0.2872(5)$ & $1.1182(3)$ & $0.6759(2)$ & $0.0165(6)$ \\
\hline $\mathrm{O} 2$ & $-0.1411(5)$ & $1.1268(3)$ & $0.6924(2)$ & $0.0157(6)$ \\
\hline N1 & $0.1784(6)$ & 0.6688 (4) & $0.5445(3)$ & $0.0127(7)$ \\
\hline $\mathrm{H} 1 \mathrm{~N}$ & $0.317(7)$ & 0.617 (4) & $0.567(4)$ & $0.015^{*}$ \\
\hline $\mathrm{H} 2 \mathrm{~N}$ & $0.169(7)$ & $0.714(4)$ & $0.468(4)$ & $0.015^{*}$ \\
\hline $\mathrm{H} 3 \mathrm{~N}$ & $0.042(7)$ & $0.632(4)$ & $0.563(3)$ & $0.015^{*}$ \\
\hline N2 & $0.1152(5)$ & $0.9858(3)$ & $0.8748(3)$ & $0.0117(7)$ \\
\hline N3 & $0.5180(5)$ & $0.8604(3)$ & $0.9033(3)$ & $0.0140(7)$ \\
\hline N4 & $0.3154(6)$ & 0.8837 (3) & $1.0623(3)$ & $0.0122(7)$ \\
\hline $\mathrm{H} 4 \mathrm{~N}$ & $0.175(7)$ & $0.920(4)$ & $1.083(3)$ & $0.015^{*}$ \\
\hline $\mathrm{C} 1$ & $0.1645(6)$ & $0.7559(4)$ & 0.5968 (3) & $0.0111(8)$ \\
\hline $\mathrm{C} 2$ & $0.3396(7)$ & $0.8239(4)$ & $0.5738(3)$ & $0.0142(8)$ \\
\hline $\mathrm{H} 2$ & 0.4666 & 0.8123 & 0.5265 & $0.017^{*}$ \\
\hline $\mathrm{C} 3$ & $0.3271(7)$ & $0.9083(4)$ & $0.6202(3)$ & $0.0128(8)$ \\
\hline H3 & 0.4488 & 0.9533 & 0.6080 & $0.015^{*}$ \\
\hline $\mathrm{C} 4$ & $0.1344(6)$ & 0.9274 (4) & $0.6853(3)$ & $0.0114(8)$ \\
\hline $\mathrm{C} 5$ & $-0.0410(6)$ & 0.8603 (4) & $0.7065(3)$ & $0.0142(8)$ \\
\hline H5 & -0.1718 & 0.8743 & 0.7507 & $0.017^{*}$ \\
\hline C6 & $-0.0248(7)$ & $0.7721(4)$ & $0.6625(3)$ & $0.0144(8)$ \\
\hline H6 & -0.1423 & 0.7239 & 0.6776 & $0.017^{*}$ \\
\hline $\mathrm{C} 7$ & $0.3204(7)$ & $0.9096(4)$ & $0.9434(3)$ & $0.0119(8)$ \\
\hline $\mathrm{C} 8$ & $0.7027(7)$ & 0.7845 (4) & $0.9855(4)$ & $0.0179(9)$ \\
\hline $\mathrm{H} 8$ & 0.8417 & 0.7468 & 0.9596 & $0.022 *$ \\
\hline C9 & $0.7061(7)$ & $0.7567(4)$ & 1.1064 (4) & $0.0185(9)$ \\
\hline H9 & 0.8443 & 0.7036 & 1.1607 & $0.022 *$ \\
\hline $\mathrm{C} 10$ & $0.5050(7)$ & 0.8081 (4) & 1.1447 (4) & $0.0152(9)$ \\
\hline H10 & 0.4981 & 0.7913 & 1.2267 & $0.018^{*}$ \\
\hline
\end{tabular}

Atomic displacement parameters $\left(A^{2}\right)$

\begin{tabular}{lllllll}
\hline & $U^{11}$ & $U^{22}$ & $U^{33}$ & $U^{12}$ & $U^{13}$ & $U^{23}$ \\
\hline $\mathrm{I} 1$ & $0.01540(15)$ & $0.01568(15)$ & $0.01591(15)$ & $-0.00270(10)$ & $0.00046(10)$ & $-0.00950(11)$ \\
$\mathrm{I} 2$ & $0.03577(19)$ & $0.02479(18)$ & $0.02029(17)$ & $-0.01282(14)$ & $0.00720(12)$ & $-0.01083(14)$ \\
$\mathrm{S} 1$ & $0.0154(5)$ & $0.0133(5)$ & $0.0099(5)$ & $-0.0035(4)$ & $0.0020(4)$ & $-0.0073(4)$ \\
$\mathrm{O} 1$ & $0.0222(16)$ & $0.0174(16)$ & $0.0158(15)$ & $-0.0127(12)$ & $0.0074(11)$ & $-0.0097(13)$ \\
$\mathrm{O} 2$ & $0.0175(15)$ & $0.0151(16)$ & $0.0119(15)$ & $0.0021(12)$ & $-0.0028(10)$ & $-0.0067(12)$ \\
$\mathrm{N} 1$ & $0.0110(18)$ & $0.0147(19)$ & $0.0125(18)$ & $0.0005(14)$ & $-0.0018(13)$ & $-0.0081(15)$ \\
$\mathrm{N} 2$ & $0.0132(17)$ & $0.0140(18)$ & $0.0094(16)$ & $-0.0020(13)$ & $0.0005(12)$ & $-0.0074(14)$ \\
$\mathrm{N} 3$ & $0.0132(18)$ & $0.0166(19)$ & $0.0118(18)$ & $-0.0009(14)$ & $0.0011(12)$ & $-0.0076(15)$ \\
$\mathrm{N} 4$ & $0.0144(18)$ & $0.0147(19)$ & $0.0114(17)$ & $-0.0054(14)$ & $0.0027(13)$ & $-0.0089(15)$ \\
$\mathrm{C} 1$ & $0.012(2)$ & $0.011(2)$ & $0.0091(19)$ & $0.0011(15)$ & $-0.0016(14)$ & $-0.0063(16)$ \\
$\mathrm{C} 2$ & $0.014(2)$ & $0.017(2)$ & $0.013(2)$ & $-0.0025(16)$ & $0.0046(15)$ & $-0.0091(17)$
\end{tabular}




\begin{tabular}{lllllll} 
C3 & $0.011(2)$ & $0.017(2)$ & $0.012(2)$ & $-0.0057(16)$ & $0.0021(14)$ & $-0.0076(17)$ \\
C4 & $0.011(2)$ & $0.012(2)$ & $0.0093(19)$ & $-0.0020(15)$ & $-0.0018(14)$ & $-0.0040(16)$ \\
C5 & $0.010(2)$ & $0.022(2)$ & $0.012(2)$ & $-0.0035(16)$ & $0.0032(14)$ & $-0.0097(18)$ \\
C6 & $0.011(2)$ & $0.017(2)$ & $0.016(2)$ & $-0.0061(16)$ & $0.0014(15)$ & $-0.0080(18)$ \\
C7 & $0.015(2)$ & $0.010(2)$ & $0.012(2)$ & $-0.0041(15)$ & $0.0003(14)$ & $-0.0060(16)$ \\
C8 & $0.016(2)$ & $0.018(2)$ & $0.019(2)$ & $-0.0030(17)$ & $0.0023(16)$ & $-0.0100(19)$ \\
C9 & $0.017(2)$ & $0.019(2)$ & $0.017(2)$ & $-0.0013(17)$ & $-0.0020(16)$ & $-0.0078(18)$ \\
C10 & $0.018(2)$ & $0.015(2)$ & $0.013(2)$ & $-0.0064(17)$ & $0.0000(15)$ & $-0.0065(17)$ \\
\hline
\end{tabular}

Geometric parameters $\left(\AA,{ }^{o}\right)$

\begin{tabular}{|c|c|c|c|}
\hline $\mathrm{I} 1-\mathrm{I} 2$ & 3.3647 (4) & $\mathrm{C} 1-\mathrm{C} 6$ & $1.376(5)$ \\
\hline $\mathrm{I} 2-\mathrm{I} 2^{\mathrm{i}}$ & $2.7623(6)$ & $\mathrm{C} 1-\mathrm{C} 2$ & $1.384(5)$ \\
\hline $\mathrm{S} 1-\mathrm{O} 1$ & $1.443(3)$ & $\mathrm{C} 2-\mathrm{C} 3$ & $1.373(5)$ \\
\hline $\mathrm{S} 1-\mathrm{O} 2$ & $1.455(3)$ & $\mathrm{C} 2-\mathrm{H} 2$ & 0.9500 \\
\hline $\mathrm{S} 1-\mathrm{N} 2$ & $1.598(3)$ & $\mathrm{C} 3-\mathrm{C} 4$ & $1.394(5)$ \\
\hline $\mathrm{S} 1-\mathrm{C} 4$ & $1.771(4)$ & $\mathrm{C} 3-\mathrm{H} 3$ & 0.9500 \\
\hline $\mathrm{N} 1-\mathrm{C} 1$ & $1.460(5)$ & $\mathrm{C} 4-\mathrm{C} 5$ & $1.382(5)$ \\
\hline $\mathrm{N} 1-\mathrm{H} 1 \mathrm{~N}$ & $0.85(4)$ & $\mathrm{C} 5-\mathrm{C} 6$ & $1.388(5)$ \\
\hline $\mathrm{N} 1-\mathrm{H} 2 \mathrm{~N}$ & $0.84(4)$ & $\mathrm{C} 5-\mathrm{H} 5$ & 0.9500 \\
\hline $\mathrm{N} 1-\mathrm{H} 3 \mathrm{~N}$ & $0.96(4)$ & $\mathrm{C} 6-\mathrm{H} 6$ & 0.9500 \\
\hline N2-C7 & $1.347(5)$ & $\mathrm{C} 8-\mathrm{C} 9$ & $1.388(5)$ \\
\hline $\mathrm{N} 3-\mathrm{C} 8$ & $1.334(5)$ & $\mathrm{C} 8-\mathrm{H} 8$ & 0.9500 \\
\hline $\mathrm{N} 3-\mathrm{C} 7$ & $1.350(5)$ & $\mathrm{C} 9-\mathrm{C} 10$ & $1.364(5)$ \\
\hline $\mathrm{N} 4-\mathrm{C} 10$ & $1.353(5)$ & C9-H9 & 0.9500 \\
\hline $\mathrm{N} 4-\mathrm{C} 7$ & $1.371(5)$ & $\mathrm{C} 10-\mathrm{H} 10$ & 0.9500 \\
\hline $\mathrm{N} 4-\mathrm{H} 4 \mathrm{~N}$ & $0.91(4)$ & & \\
\hline 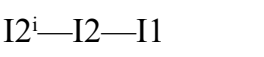 & $174.992(16)$ & $\mathrm{C} 2-\mathrm{C} 3-\mathrm{C} 4$ & $119.5(3)$ \\
\hline $\mathrm{O} 1-\mathrm{S} 1-\mathrm{O} 2$ & $115.97(17)$ & $\mathrm{C} 2-\mathrm{C} 3-\mathrm{H} 3$ & 120.3 \\
\hline $\mathrm{O} 1-\mathrm{S} 1-\mathrm{N} 2$ & $114.37(16)$ & $\mathrm{C} 4-\mathrm{C} 3-\mathrm{H} 3$ & 120.3 \\
\hline $\mathrm{O} 2-\mathrm{S} 1-\mathrm{N} 2$ & $103.10(16)$ & $\mathrm{C} 5-\mathrm{C} 4-\mathrm{C} 3$ & $121.0(4)$ \\
\hline $\mathrm{O} 1-\mathrm{S} 1-\mathrm{C} 4$ & $107.10(17)$ & $\mathrm{C} 5-\mathrm{C} 4-\mathrm{S} 1$ & $120.1(3)$ \\
\hline $\mathrm{O} 2-\mathrm{S} 1-\mathrm{C} 4$ & $105.90(16)$ & $\mathrm{C} 3-\mathrm{C} 4-\mathrm{S} 1$ & $118.7(3)$ \\
\hline $\mathrm{N} 2-\mathrm{S} 1-\mathrm{C} 4$ & $110.06(17)$ & $\mathrm{C} 4-\mathrm{C} 5-\mathrm{C} 6$ & $119.5(4)$ \\
\hline $\mathrm{C} 1-\mathrm{N} 1-\mathrm{H} 1 \mathrm{~N}$ & $107(3)$ & $\mathrm{C} 4-\mathrm{C} 5-\mathrm{H} 5$ & 120.2 \\
\hline $\mathrm{C} 1-\mathrm{N} 1-\mathrm{H} 2 \mathrm{~N}$ & $108(3)$ & $\mathrm{C} 6-\mathrm{C} 5-\mathrm{H} 5$ & 120.2 \\
\hline $\mathrm{H} 1 \mathrm{~N}-\mathrm{N} 1-\mathrm{H} 2 \mathrm{~N}$ & $107(4)$ & $\mathrm{C} 1-\mathrm{C} 6-\mathrm{C} 5$ & $118.7(3)$ \\
\hline $\mathrm{C} 1-\mathrm{N} 1-\mathrm{H} 3 \mathrm{~N}$ & $110(2)$ & $\mathrm{C} 1-\mathrm{C} 6-\mathrm{H} 6$ & 120.6 \\
\hline $\mathrm{H} 1 \mathrm{~N}-\mathrm{N} 1-\mathrm{H} 3 \mathrm{~N}$ & $118(4)$ & $\mathrm{C} 5-\mathrm{C} 6-\mathrm{H} 6$ & 120.6 \\
\hline $\mathrm{H} 2 \mathrm{~N}-\mathrm{N} 1-\mathrm{H} 3 \mathrm{~N}$ & $106(3)$ & $\mathrm{N} 2-\mathrm{C} 7-\mathrm{N} 3$ & $125.1(4)$ \\
\hline $\mathrm{C} 7-\mathrm{N} 2-\mathrm{S} 1$ & $120.8(3)$ & $\mathrm{N} 2-\mathrm{C} 7-\mathrm{N} 4$ & $114.0(3)$ \\
\hline $\mathrm{C} 8-\mathrm{N} 3-\mathrm{C} 7$ & $116.5(3)$ & $\mathrm{N} 3-\mathrm{C} 7-\mathrm{N} 4$ & $120.8(3)$ \\
\hline $\mathrm{C} 10-\mathrm{N} 4-\mathrm{C} 7$ & $122.3(3)$ & $\mathrm{N} 3-\mathrm{C} 8-\mathrm{C} 9$ & $124.6(4)$ \\
\hline $\mathrm{C} 10-\mathrm{N} 4-\mathrm{H} 4 \mathrm{~N}$ & $122(2)$ & $\mathrm{N} 3-\mathrm{C} 8-\mathrm{H} 8$ & 117.7 \\
\hline $\mathrm{C} 7-\mathrm{N} 4-\mathrm{H} 4 \mathrm{~N}$ & $116(2)$ & $\mathrm{C} 9-\mathrm{C} 8-\mathrm{H} 8$ & 117.7 \\
\hline $\mathrm{C} 6-\mathrm{C} 1-\mathrm{C} 2$ & $122.2(4)$ & $\mathrm{C} 10-\mathrm{C} 9-\mathrm{C} 8$ & $117.9(4)$ \\
\hline $\mathrm{C} 6-\mathrm{C} 1-\mathrm{N} 1$ & $119.6(3)$ & $\mathrm{C} 10-\mathrm{C} 9-\mathrm{H} 9$ & 121.0 \\
\hline
\end{tabular}




$\begin{array}{ll}\mathrm{C} 2-\mathrm{C} 1-\mathrm{N} 1 & 118.1(3) \\ \mathrm{C} 3-\mathrm{C} 2-\mathrm{C} 1 & 119.0(4) \\ \mathrm{C} 3-\mathrm{C} 2-\mathrm{H} 2 & 120.5 \\ \mathrm{C} 1-\mathrm{C} 2-\mathrm{H} 2 & 120.5 \\ & \\ \mathrm{O} 1-\mathrm{S} 1-\mathrm{N} 2-\mathrm{C} 7 & -50.9(3) \\ \mathrm{O} 2-\mathrm{S} 1-\mathrm{N} 2-\mathrm{C} 7 & -177.7(3) \\ \mathrm{C} 4-\mathrm{S} 1-\mathrm{N} 2-\mathrm{C} 7 & 69.7(3) \\ \mathrm{C} 6-\mathrm{C} 1-\mathrm{C} 2-\mathrm{C} 3 & 1.4(6) \\ \mathrm{N} 1-\mathrm{C} 1-\mathrm{C} 2-\mathrm{C} 3 & 178.7(4) \\ \mathrm{C} 1-\mathrm{C} 2-\mathrm{C} 3-\mathrm{C} 4 & -2.4(6) \\ \mathrm{C} 2-\mathrm{C} 3-\mathrm{C} 4-\mathrm{C} 5 & 1.6(6) \\ \mathrm{C} 2-\mathrm{C} 3-\mathrm{C} 4-\mathrm{S} 1 & -173.2(3) \\ \mathrm{O} 1-\mathrm{S} 1-\mathrm{C} 4-\mathrm{C} 5 & -169.1(3) \\ \mathrm{O} 2-\mathrm{S} 1-\mathrm{C} 4-\mathrm{C} 5 & -44.7(3) \\ \mathrm{N} 2-\mathrm{S} 1-\mathrm{C} 4-\mathrm{C} 5 & 66.0(3) \\ \mathrm{O} 1-\mathrm{S} 1-\mathrm{C} 4-\mathrm{C} 3 & 5.8(4) \\ \mathrm{O} 2-\mathrm{S} 1-\mathrm{C} 4-\mathrm{C} 3 & 130.1(3) \\ \mathrm{N} 2-\mathrm{S} 1-\mathrm{C} 4-\mathrm{C} 3 & -119.1(3) \\ \mathrm{C} 3-\mathrm{C} 4-\mathrm{C} 5-\mathrm{C} 6 & 0.2(6)\end{array}$

C8-C9- $\mathrm{H} 9$

$\mathrm{N} 4-\mathrm{C} 10-\mathrm{C} 9$

$\mathrm{N} 4-\mathrm{C} 10-\mathrm{H} 10$

C9- $\mathrm{C} 10-\mathrm{H} 10$

$\mathrm{S} 1-\mathrm{C} 4-\mathrm{C} 5-\mathrm{C} 6$

$\mathrm{C} 2-\mathrm{C} 1-\mathrm{C} 6-\mathrm{C} 5$

$\mathrm{N} 1-\mathrm{C} 1-\mathrm{C} 6-\mathrm{C} 5$

$\mathrm{C} 4-\mathrm{C} 5-\mathrm{C} 6-\mathrm{C} 1$

$\mathrm{S} 1-\mathrm{N} 2-\mathrm{C} 7-\mathrm{N} 3$

$\mathrm{S} 1-\mathrm{N} 2-\mathrm{C} 7-\mathrm{N} 4$

$\mathrm{C} 8-\mathrm{N} 3-\mathrm{C} 7-\mathrm{N} 2$

$\mathrm{C} 8-\mathrm{N} 3-\mathrm{C} 7-\mathrm{N} 4$

$\mathrm{C} 10-\mathrm{N} 4-\mathrm{C} 7-\mathrm{N} 2$

$\mathrm{C} 10-\mathrm{N} 4-\mathrm{C} 7-\mathrm{N} 3$

$\mathrm{C} 7-\mathrm{N} 3-\mathrm{C} 8-\mathrm{C} 9$

N3-C8-C9-C10

C7- $44-\mathrm{C} 10-\mathrm{C} 9$

$\mathrm{C} 8-\mathrm{C} 9-\mathrm{C} 10-\mathrm{N} 4$
121.0

117.9 (4)

121.1

121.1

$174.9(3)$

$0.4(6)$

$-176.9(3)$

$-1.2(6)$

$-12.7(5)$

$167.5(3)$

$-178.6(4)$

$1.2(5)$

$179.5(3)$

$-0.3(5)$

$-1.9(6)$

$1.6(6)$

$-0.1(5)$

$-0.5(6)$

Symmetry code: (i) $-x-1,-y+1,-z+2$.

Hydrogen-bond geometry $\left(A,{ }^{\circ}\right)$

\begin{tabular}{lllll}
\hline$D-\mathrm{H} \cdots A$ & $D-\mathrm{H}$ & $\mathrm{H} \cdots A$ & $D \cdots A$ & $D-\mathrm{H} \cdots A$ \\
\hline $\mathrm{N} 1-\mathrm{H} 1 N \cdots \mathrm{I} 1^{\mathrm{ii}}$ & $0.85(4)$ & $2.64(4)$ & $3.482(4)$ & $172(4)$ \\
$\mathrm{N} 1-\mathrm{H} 2 N \cdots \mathrm{O} 2^{\mathrm{iii}}$ & $0.84(4)$ & $2.00(4)$ & $2.798(4)$ & $158(4)$ \\
$\mathrm{N} 1-\mathrm{H} 3 N \cdots \mathrm{I} 1$ & $0.96(4)$ & $2.59(4)$ & $3.524(3)$ & $165(3)$ \\
$\mathrm{N} 4-\mathrm{H} 4 N \cdots \mathrm{N} 2^{2 \mathrm{iv}}$ & $0.91(4)$ & $2.00(4)$ & $2.906(5)$ & $174(4)$ \\
\hline
\end{tabular}

Symmetry codes: (ii) $x+1, y, z$; (iii) $-x,-y+2,-z+1$; (iv) $-x,-y+2,-z+2$.

\section{(V) 2-\{[(4-Azaniumylphenyl)sulfonyl]azanidyl\}pyrimidin-1-ium tetrafluoroborate monohydrate}

Crystal data

$\mathrm{C}_{10} \mathrm{H}_{11} \mathrm{~N}_{4} \mathrm{O}_{2} \mathrm{~S}^{+} \cdot \mathrm{BF}_{4}^{--} \cdot \mathrm{H}_{2} \mathrm{O}$

$M_{r}=356.11$

Monoclinic, $P 2_{1} / n$

Hall symbol: -P 2 yn

$a=5.8539$ (1) $\AA$

$b=11.3629(3) \AA$

$c=21.2080(6) \AA$

$\beta=90.982(2)^{\circ}$

$V=1410.49(6) \AA^{3}$

$Z=4$

\section{Data collection}

Oxford Diffraction Xcalibur E diffractometer

Radiation source: fine-focus sealed tube Graphite monochromator
$F(000)=728$

$D_{\mathrm{x}}=1.677 \mathrm{Mg} \mathrm{m}^{-3}$

Mo $K \alpha$ radiation, $\lambda=0.71073 \AA$

Cell parameters from 4378 reflections

$\theta=3.4-29.8^{\circ}$

$\mu=0.30 \mathrm{~mm}^{-1}$

$T=123 \mathrm{~K}$

Fragment, light yellow

$0.20 \times 0.12 \times 0.05 \mathrm{~mm}$

$\omega$ scans

Absorption correction: multi-scan

(CrysAlis PRO; Oxford Diffraction, 2010)

$T_{\min }=0.874, T_{\max }=1.000$ 
8710 measured reflections 3549 independent reflections 2955 reflections with $I>2 \sigma(I)$

$R_{\text {int }}=0.024$

\section{Refinement}

Refinement on $F^{2}$

Least-squares matrix: full

$R\left[F^{2}>2 \sigma\left(F^{2}\right)\right]=0.037$

$w R\left(F^{2}\right)=0.089$

$S=1.03$

3549 reflections

232 parameters

3 restraints

Primary atom site location: structure-invariant direct methods $\theta_{\max }=29.0^{\circ}, \theta_{\min }=3.4^{\circ}$

$h=-7 \rightarrow 7$

$k=-15 \rightarrow 11$

$l=-24 \rightarrow 27$

Secondary atom site location: difference Fourier map

Hydrogen site location: inferred from neighbouring sites

$\mathrm{H}$ atoms treated by a mixture of independent and constrained refinement

$w=1 /\left[\sigma^{2}\left(F_{\mathrm{o}}^{2}\right)+(0.0362 P)^{2}+0.6973 P\right]$ where $P=\left(F_{\mathrm{o}}^{2}+2 F_{\mathrm{c}}^{2}\right) / 3$

$(\Delta / \sigma)_{\max }=0.003$

$\Delta \rho_{\max }=0.35 \mathrm{e} \AA^{-3}$

$\Delta \rho_{\min }=-0.46$ e $\AA^{-3}$

\section{Special details}

Experimental. Absorption correction: CrysAlisPro, Oxford Diffraction Ltd., Version 1.171.34.40 (release 27-08-2010 CrysAlis171 .NET) (compiled Aug 27 2010,11:50:40) Empirical absorption correction using spherical harmonics, implemented in SCALE3 ABSPACK scaling algorithm.

Geometry. All s.u.'s (except the s.u. in the dihedral angle between two 1.s. planes) are estimated using the full covariance matrix. The cell s.u.'s are taken into account individually in the estimation of s.u.'s in distances, angles and torsion angles; correlations between s.u.'s in cell parameters are only used when they are defined by crystal symmetry. An approximate (isotropic) treatment of cell s.u.'s is used for estimating s.u.'s involving 1.s. planes.

Refinement. Refinement of $F^{2}$ against ALL reflections. The weighted $R$-factor $w R$ and goodness of fit $S$ are based on $F^{2}$, conventional $R$-factors $R$ are based on $F$, with $F$ set to zero for negative $F^{2}$. The threshold expression of $F^{2}>2 \sigma\left(F^{2}\right)$ is used only for calculating $R$-factors (gt) etc. and is not relevant to the choice of reflections for refinement. $R$-factors based on $F^{2}$ are statistically about twice as large as those based on $F$, and $R$ - factors based on ALL data will be even larger.

Fractional atomic coordinates and isotropic or equivalent isotropic displacement parameters $\left(\AA^{2}\right)$

\begin{tabular}{lllll}
\hline & $x$ & $y$ & $z$ & $U_{\text {iso }} * / U_{\text {eq }}$ \\
\hline S1 & $0.21736(6)$ & $0.24493(3)$ & $1.039691(17)$ & $0.01188(10)$ \\
F1 & $-0.15946(17)$ & $0.20817(10)$ & $0.74824(5)$ & $0.0301(3)$ \\
F2 & $0.09745(19)$ & $0.18041(11)$ & $0.82770(5)$ & $0.0312(3)$ \\
F3 & $0.2036(2)$ & $0.15335(12)$ & $0.72644(6)$ & $0.0434(3)$ \\
F4 & $0.13039(17)$ & $0.33817(10)$ & $0.76257(5)$ & $0.0273(3)$ \\
O1 & $0.44811(18)$ & $0.27762(10)$ & $1.05680(5)$ & $0.0175(2)$ \\
O2 & $0.05509(19)$ & $0.25011(10)$ & $1.09069(5)$ & $0.0164(2)$ \\
O1W & $-0.4027(2)$ & $0.41744(12)$ & $0.76066(6)$ & $0.0245(3)$ \\
N1 & $-0.0902(2)$ & $0.55457(13)$ & $0.82772(7)$ & $0.0143(3)$ \\
N2 & $0.1822(2)$ & $0.11597(11)$ & $1.01075(6)$ & $0.0120(3)$ \\
N3 & $0.2260(2)$ & $-0.01928(12)$ & $0.93253(6)$ & $0.0126(3)$ \\
N4 & $0.4790(2)$ & $0.14123(12)$ & $0.93657(6)$ & $0.0156(3)$ \\
C1 & $-0.0140(2)$ & $0.47962(14)$ & $0.88004(7)$ & $0.0117(3)$ \\
C2 & $0.2045(3)$ & $0.49308(14)$ & $0.90438(8)$ & $0.0157(3)$ \\
H2 & 0.3052 & 0.5501 & 0.8875 & $0.019 *$ \\
C3 & $0.2735(2)$ & $0.42144(14)$ & $0.95408(7)$ & $0.0145(3)$ \\
H3 & 0.4245 & 0.4276 & 0.9709 & $0.017 *$
\end{tabular}




$\begin{array}{lllll}\text { C4 } & 0.1225(2) & 0.34096(13) & 0.97914(7) & 0.0115(3) \\ \text { C5 } & -0.0992(2) & 0.33031(14) & 0.95543(7) & 0.0143(3) \\ \text { H5 } & -0.2030 & 0.2765 & 0.9737 & 0.017^{*} \\ \text { C6 } & -0.1662(2) & 0.39939(14) & 0.90476(7) & 0.0143(3) \\ \text { H6 } & -0.3157 & 0.3919 & 0.8870 & 0.017^{*} \\ \text { C7 } & 0.2996(2) & 0.08243(14) & 0.95992(7) & 0.0114(3) \\ \text { C8 } & 0.3297(3) & -0.06628(15) & 0.88228(7) & 0.0153(3) \\ \text { H8 } & 0.2755 & -0.1376 & 0.8641 & 0.018^{*} \\ \text { C9 } & 0.5143(3) & -0.00977(15) & 0.85782(8) & 0.0177(3) \\ \text { H9 } & 0.5934 & -0.0405 & 0.8227 & 0.021^{*} \\ \text { C10 } & 0.5806(3) & 0.09446(16) & 0.88665(8) & 0.0184(3) \\ \text { H10 } & 0.7071 & 0.1354 & 0.8697 & 0.022^{*} \\ \text { B1 } & 0.0703(3) & 0.21971(18) & 0.76723(9) & 0.0183(4) \\ \text { H1N } & 0.030(4) & 0.5713(19) & 0.8011(10) & 0.029(6)^{*} \\ \text { H2N } & -0.133(3) & 0.623(2) & 0.8436(9) & 0.025(5)^{*} \\ \text { H3N } & -0.206(4) & 0.520(2) & 0.8040(11) & 0.040(6)^{*} \\ \text { H4N } & 0.103(3) & -0.0515(17) & 0.9493(9) & 0.023(5)^{*} \\ \text { H1W } & -0.5492(16) & 0.407(2) & 0.7599(12) & 0.060(8)^{*} \\ \text { H2W } & -0.355(4) & 0.3449(11) & 0.7561(12) & 0.053(8)^{*}\end{array}$

Atomic displacement parameters $\left(\AA^{2}\right)$

\begin{tabular}{lllllll}
\hline & $U^{11}$ & $U^{22}$ & $U^{33}$ & $U^{12}$ & $U^{13}$ & $U^{23}$ \\
\hline S1 & $0.01499(17)$ & $0.00899(19)$ & $0.01162(19)$ & $-0.00181(13)$ & $-0.00057(13)$ & $-0.00011(15)$ \\
F1 & $0.0210(5)$ & $0.0234(6)$ & $0.0456(7)$ & $0.0003(4)$ & $-0.0106(5)$ & $-0.0098(5)$ \\
F2 & $0.0366(6)$ & $0.0335(7)$ & $0.0236(6)$ & $0.0045(5)$ & $0.0003(4)$ & $0.0068(5)$ \\
F3 & $0.0405(7)$ & $0.0512(8)$ & $0.0388(7)$ & $0.0177(6)$ & $0.0144(5)$ & $-0.0141(6)$ \\
F4 & $0.0243(5)$ & $0.0253(6)$ & $0.0322(6)$ & $-0.0070(4)$ & $-0.0003(4)$ & $0.0046(5)$ \\
O1 & $0.0184(5)$ & $0.0145(6)$ & $0.0194(6)$ & $-0.0038(4)$ & $-0.0066(4)$ & $0.0021(5)$ \\
O2 & $0.0242(6)$ & $0.0122(6)$ & $0.0131(5)$ & $-0.0013(4)$ & $0.0037(4)$ & $-0.0010(5)$ \\
O1W & $0.0182(6)$ & $0.0221(7)$ & $0.0331(7)$ & $-0.0004(5)$ & $-0.0037(5)$ & $-0.0056(6)$ \\
N1 & $0.0184(6)$ & $0.0114(7)$ & $0.0132(7)$ & $0.0001(5)$ & $0.0005(5)$ & $0.0014(6)$ \\
N2 & $0.0154(6)$ & $0.0079(6)$ & $0.0128(6)$ & $-0.0026(5)$ & $0.0016(5)$ & $-0.0002(5)$ \\
N3 & $0.0143(6)$ & $0.0100(6)$ & $0.0135(6)$ & $-0.0008(5)$ & $0.0010(5)$ & $0.0010(5)$ \\
N4 & $0.0149(6)$ & $0.0153(7)$ & $0.0166(7)$ & $-0.0036(5)$ & $0.0026(5)$ & $0.0006(6)$ \\
C1 & $0.0165(7)$ & $0.0098(7)$ & $0.0087(7)$ & $0.0011(6)$ & $0.0011(5)$ & $0.0001(6)$ \\
C2 & $0.0166(7)$ & $0.0122(8)$ & $0.0182(8)$ & $-0.0046(6)$ & $0.0018(6)$ & $0.0010(7)$ \\
C3 & $0.0131(6)$ & $0.0134(8)$ & $0.0169(8)$ & $-0.0031(6)$ & $-0.0013(5)$ & $-0.0006(7)$ \\
C4 & $0.0146(7)$ & $0.0090(7)$ & $0.0110(7)$ & $-0.0002(5)$ & $0.0009(5)$ & $-0.0012(6)$ \\
C5 & $0.0136(7)$ & $0.0133(8)$ & $0.0160(7)$ & $-0.0033(6)$ & $0.0020(5)$ & $0.0010(7)$ \\
C6 & $0.0123(6)$ & $0.0155(8)$ & $0.0152(7)$ & $-0.0009(6)$ & $0.0002(5)$ & $0.0003(7)$ \\
C7 & $0.0122(6)$ & $0.0097(7)$ & $0.0122(7)$ & $0.0005(5)$ & $-0.0023(5)$ & $0.0006(6)$ \\
C8 & $0.0202(7)$ & $0.0123(8)$ & $0.0135(7)$ & $0.0025(6)$ & $0.0001(6)$ & $-0.0007(7)$ \\
C9 & $0.0198(7)$ & $0.0193(9)$ & $0.0142(8)$ & $0.0023(6)$ & $0.0037(6)$ & $-0.0017(7)$ \\
C10 & $0.0157(7)$ & $0.0218(9)$ & $0.0179(8)$ & $-0.0019(6)$ & $0.0032(6)$ & $0.0020(7)$ \\
B1 & $0.0155(8)$ & $0.0201(10)$ & $0.0194(9)$ & $0.0029(7)$ & $0.0023(6)$ & $-0.0027(8)$ \\
& & & & & & \\
\hline
\end{tabular}


Geometric parameters $\left(A,{ }^{\circ}\right)$

\begin{tabular}{|c|c|c|c|}
\hline $\mathrm{S} 1-\mathrm{O} 1$ & $1.4413(11)$ & $\mathrm{N} 4-\mathrm{C} 10$ & $1.334(2)$ \\
\hline $\mathrm{S} 1-\mathrm{O} 2$ & $1.4528(11)$ & $\mathrm{N} 4-\mathrm{C} 7$ & $1.3463(19)$ \\
\hline $\mathrm{S} 1-\mathrm{N} 2$ & $1.6006(13)$ & $\mathrm{C} 1-\mathrm{C} 2$ & $1.379(2)$ \\
\hline $\mathrm{S} 1-\mathrm{C} 4$ & $1.7675(16)$ & $\mathrm{C} 1-\mathrm{C} 6$ & $1.384(2)$ \\
\hline $\mathrm{F} 1-\mathrm{B} 1$ & $1.404(2)$ & $\mathrm{C} 2-\mathrm{C} 3$ & $1.386(2)$ \\
\hline $\mathrm{F} 2-\mathrm{B} 1$ & $1.365(2)$ & $\mathrm{C} 2-\mathrm{H} 2$ & 0.9500 \\
\hline $\mathrm{F} 3-\mathrm{B} 1$ & $1.396(2)$ & $\mathrm{C} 3-\mathrm{C} 4$ & $1.384(2)$ \\
\hline $\mathrm{F} 4-\mathrm{B} 1$ & $1.395(2)$ & $\mathrm{C} 3-\mathrm{H} 3$ & 0.9500 \\
\hline $\mathrm{O} 1 \mathrm{~W}-\mathrm{H} 1 \mathrm{~W}$ & $0.867(10)$ & $\mathrm{C} 4-\mathrm{C} 5$ & $1.389(2)$ \\
\hline $\mathrm{O} 1 \mathrm{~W}-\mathrm{H} 2 \mathrm{~W}$ & $0.877(9)$ & $\mathrm{C} 5-\mathrm{C} 6$ & $1.382(2)$ \\
\hline $\mathrm{N} 1-\mathrm{C} 1$ & $1.463(2)$ & $\mathrm{C} 5-\mathrm{H} 5$ & 0.9500 \\
\hline $\mathrm{N} 1-\mathrm{H} 1 \mathrm{~N}$ & $0.93(2)$ & $\mathrm{C} 6-\mathrm{H} 6$ & 0.9500 \\
\hline $\mathrm{N} 1-\mathrm{H} 2 \mathrm{~N}$ & $0.88(2)$ & $\mathrm{C} 8-\mathrm{C} 9$ & $1.367(2)$ \\
\hline $\mathrm{N} 1-\mathrm{H} 3 \mathrm{~N}$ & $0.92(2)$ & $\mathrm{C} 8-\mathrm{H} 8$ & 0.9500 \\
\hline $\mathrm{N} 2-\mathrm{C} 7$ & $1.3433(18)$ & $\mathrm{C} 9-\mathrm{C} 10$ & $1.385(2)$ \\
\hline $\mathrm{N} 3-\mathrm{C} 8$ & $1.3460(19)$ & C9- $\mathrm{H} 9$ & 0.9500 \\
\hline $\mathrm{N} 3-\mathrm{C} 7$ & $1.360(2)$ & $\mathrm{C} 10-\mathrm{H} 10$ & 0.9500 \\
\hline $\mathrm{N} 3-\mathrm{H} 4 \mathrm{~N}$ & $0.89(2)$ & & \\
\hline $\mathrm{O} 1-\mathrm{S} 1-\mathrm{O} 2$ & $115.10(7)$ & $\mathrm{C} 3-\mathrm{C} 4-\mathrm{C} 5$ & $121.16(14)$ \\
\hline $\mathrm{O} 1-\mathrm{S} 1-\mathrm{N} 2$ & $116.50(7)$ & $\mathrm{C} 3-\mathrm{C} 4-\mathrm{S} 1$ & $119.46(11)$ \\
\hline $\mathrm{O} 2-\mathrm{S} 1-\mathrm{N} 2$ & $103.96(7)$ & $\mathrm{C} 5-\mathrm{C} 4-\mathrm{S} 1$ & $119.30(12)$ \\
\hline $\mathrm{O} 1-\mathrm{S} 1-\mathrm{C} 4$ & $107.78(7)$ & $\mathrm{C} 6-\mathrm{C} 5-\mathrm{C} 4$ & $118.88(14)$ \\
\hline $\mathrm{O} 2-\mathrm{S} 1-\mathrm{C} 4$ & $108.33(7)$ & $\mathrm{C} 6-\mathrm{C} 5-\mathrm{H} 5$ & 120.6 \\
\hline $\mathrm{N} 2-\mathrm{S} 1-\mathrm{C} 4$ & $104.47(7)$ & $\mathrm{C} 4-\mathrm{C} 5-\mathrm{H} 5$ & 120.6 \\
\hline $\mathrm{H} 1 \mathrm{~W}-\mathrm{O} 1 \mathrm{~W}-\mathrm{H} 2 \mathrm{~W}$ & $100.6(17)$ & $\mathrm{C} 5-\mathrm{C} 6-\mathrm{C} 1$ & $119.49(14)$ \\
\hline $\mathrm{C} 1-\mathrm{N} 1-\mathrm{H} 1 \mathrm{~N}$ & $110.8(13)$ & $\mathrm{C} 5-\mathrm{C} 6-\mathrm{H} 6$ & 120.3 \\
\hline $\mathrm{C} 1-\mathrm{N} 1-\mathrm{H} 2 \mathrm{~N}$ & $107.8(13)$ & $\mathrm{C} 1-\mathrm{C} 6-\mathrm{H} 6$ & 120.3 \\
\hline $\mathrm{H} 1 \mathrm{~N}-\mathrm{N} 1-\mathrm{H} 2 \mathrm{~N}$ & $106.0(18)$ & $\mathrm{N} 2-\mathrm{C} 7-\mathrm{N} 4$ & $124.56(14)$ \\
\hline $\mathrm{C} 1-\mathrm{N} 1-\mathrm{H} 3 \mathrm{~N}$ & $112.4(15)$ & $\mathrm{N} 2-\mathrm{C} 7-\mathrm{N} 3$ & $114.91(13)$ \\
\hline $\mathrm{H} 1 \mathrm{~N}-\mathrm{N} 1-\mathrm{H} 3 \mathrm{~N}$ & $108.2(19)$ & $\mathrm{N} 4-\mathrm{C} 7-\mathrm{N} 3$ & $120.52(13)$ \\
\hline $\mathrm{H} 2 \mathrm{~N}-\mathrm{N} 1-\mathrm{H} 3 \mathrm{~N}$ & $111.5(19)$ & $\mathrm{N} 3-\mathrm{C} 8-\mathrm{C} 9$ & $118.98(15)$ \\
\hline $\mathrm{C} 7-\mathrm{N} 2-\mathrm{S} 1$ & $120.22(11)$ & $\mathrm{N} 3-\mathrm{C} 8-\mathrm{H} 8$ & 120.5 \\
\hline $\mathrm{C} 8-\mathrm{N} 3-\mathrm{C} 7$ & $122.10(13)$ & $\mathrm{C} 9-\mathrm{C} 8-\mathrm{H} 8$ & 120.5 \\
\hline $\mathrm{C} 8-\mathrm{N} 3-\mathrm{H} 4 \mathrm{~N}$ & $122.3(13)$ & $\mathrm{C} 8-\mathrm{C} 9-\mathrm{C} 10$ & $116.84(15)$ \\
\hline $\mathrm{C} 7-\mathrm{N} 3-\mathrm{H} 4 \mathrm{~N}$ & $115.6(13)$ & $\mathrm{C} 8-\mathrm{C} 9-\mathrm{H} 9$ & 121.6 \\
\hline $\mathrm{C} 10-\mathrm{N} 4-\mathrm{C} 7$ & $117.11(14)$ & $\mathrm{C} 10-\mathrm{C} 9-\mathrm{H} 9$ & 121.6 \\
\hline $\mathrm{C} 2-\mathrm{C} 1-\mathrm{C} 6$ & $122.02(14)$ & $\mathrm{N} 4-\mathrm{C} 10-\mathrm{C} 9$ & $124.44(15)$ \\
\hline $\mathrm{C} 2-\mathrm{C} 1-\mathrm{N} 1$ & $119.22(14)$ & $\mathrm{N} 4-\mathrm{C} 10-\mathrm{H} 10$ & 117.8 \\
\hline $\mathrm{C} 6-\mathrm{C} 1-\mathrm{N} 1$ & $118.73(13)$ & $\mathrm{C} 9-\mathrm{C} 10-\mathrm{H} 10$ & 117.8 \\
\hline $\mathrm{C} 1-\mathrm{C} 2-\mathrm{C} 3$ & $118.40(14)$ & $\mathrm{F} 2-\mathrm{B} 1-\mathrm{F} 4$ & $110.92(15)$ \\
\hline $\mathrm{C} 1-\mathrm{C} 2-\mathrm{H} 2$ & 120.8 & $\mathrm{~F} 2-\mathrm{B} 1-\mathrm{F} 3$ & $110.39(15)$ \\
\hline $\mathrm{C} 3-\mathrm{C} 2-\mathrm{H} 2$ & 120.8 & $\mathrm{~F} 4-\mathrm{B} 1-\mathrm{F} 3$ & $109.46(15)$ \\
\hline $\mathrm{C} 4-\mathrm{C} 3-\mathrm{C} 2$ & $120.00(14)$ & $\mathrm{F} 2-\mathrm{B} 1-\mathrm{F} 1$ & $109.54(14)$ \\
\hline $\mathrm{C} 4-\mathrm{C} 3-\mathrm{H} 3$ & 120.0 & $\mathrm{~F} 4-\mathrm{B} 1-\mathrm{F} 1$ & $108.13(14)$ \\
\hline $\mathrm{C} 2-\mathrm{C} 3-\mathrm{H} 3$ & 120.0 & $\mathrm{~F} 3-\mathrm{B} 1-\mathrm{F} 1$ & $108.33(15)$ \\
\hline
\end{tabular}




$\begin{array}{llll}\mathrm{O} 1-\mathrm{S} 1-\mathrm{N} 2-\mathrm{C} 7 & -55.37(14) & \mathrm{S} 1-\mathrm{C} 4-\mathrm{C} 5-\mathrm{C} 6 & -174.93(12) \\ \mathrm{O} 2-\mathrm{S} 1-\mathrm{N} 2-\mathrm{C} 7 & 176.88(12) & \mathrm{C} 4-\mathrm{C} 5-\mathrm{C} 6-\mathrm{C} 1 & -1.8(2) \\ \mathrm{C} 4-\mathrm{S} 1-\mathrm{N} 2-\mathrm{C} 7 & 63.38(13) & \mathrm{C} 2-\mathrm{C} 1-\mathrm{C} 6-\mathrm{C} 5 & 0.1(2) \\ \mathrm{C} 6-\mathrm{C} 1-\mathrm{C} 2-\mathrm{C} 3 & 1.7(2) & \mathrm{N} 1-\mathrm{C} 1-\mathrm{C} 6-\mathrm{C} 5 & -178.09(14) \\ \mathrm{N} 1-\mathrm{C} 1-\mathrm{C} 2-\mathrm{C} 3 & 179.84(14) & \mathrm{S} 1-\mathrm{N} 2-\mathrm{C} 7-\mathrm{N} 4 & 12.5(2) \\ \mathrm{C} 1-\mathrm{C} 2-\mathrm{C} 3-\mathrm{C} 4 & -1.7(2) & \mathrm{S} 1-\mathrm{N} 2-\mathrm{C} 7-\mathrm{N} 3 & -168.26(11) \\ \mathrm{C} 2-\mathrm{C} 3-\mathrm{C} 4-\mathrm{C} 5 & -0.1(2) & \mathrm{C} 10-\mathrm{N} 4-\mathrm{C} 7-\mathrm{N} 2 & 178.32(15) \\ \mathrm{C} 2-\mathrm{C} 3-\mathrm{C} 4-\mathrm{S} 1 & 176.70(12) & \mathrm{C} 10-\mathrm{N} 4-\mathrm{C} 7-\mathrm{N} 3 & -0.9(2) \\ \mathrm{O} 1-\mathrm{S} 1-\mathrm{C} 4-\mathrm{C} 3 & 5.16(15) & \mathrm{C} 8-\mathrm{N} 3-\mathrm{C} 7-\mathrm{N} 2 & -178.12(13) \\ \mathrm{O} 2-\mathrm{S} 1-\mathrm{C} 4-\mathrm{C} 3 & 130.29(13) & \mathrm{C} 8-\mathrm{N} 3-\mathrm{C} 7-\mathrm{N} 4 & -0.3(2) \\ \mathrm{N} 2-\mathrm{S} 1-\mathrm{C} 4-\mathrm{C} 3 & -119.36(13) & \mathrm{C} 7-\mathrm{N} 3-\mathrm{C} 8-\mathrm{C} 9 & -0.8(2) \\ \mathrm{O} 1-\mathrm{S} 1-\mathrm{C} 4-\mathrm{C} 5 & -178.00(12) & \mathrm{N} 3-\mathrm{C} 8-\mathrm{C} 9-\mathrm{C} 10 & -0.2(2) \\ \mathrm{O} 2-\mathrm{S} 1-\mathrm{C} 4-\mathrm{C} 5 & -52.87(14) & \mathrm{C} 7-\mathrm{N} 4-\mathrm{C} 10-\mathrm{C} 9 & 1.1(3) \\ \mathrm{N} 2-\mathrm{S} 1-\mathrm{C} 4-\mathrm{C} 5 & 57.48(14) & \mathrm{C} 8-\mathrm{C} 9-\mathrm{C} 10-\mathrm{N} 4 & \\ \mathrm{C} 3-\mathrm{C} 4-\mathrm{C} 5-\mathrm{C} 6 & 1.9(2) & & \end{array}$

Hydrogen-bond geometry $\left(\AA,{ }^{\circ}\right)$

\begin{tabular}{lllll}
\hline$D-\mathrm{H} \cdots A$ & $D-\mathrm{H}$ & $\mathrm{H} \cdots A$ & $D \cdots A$ & $D-\mathrm{H} \cdots A$ \\
\hline $\mathrm{N} 1-\mathrm{H} 1 N \cdots \mathrm{F} 3^{\mathrm{i}}$ & $0.93(2)$ & $1.92(2)$ & $2.7912(17)$ & $156.0(19)$ \\
$\mathrm{N} 1-\mathrm{H} 2 N \cdots \mathrm{O} 2^{\mathrm{ii}}$ & $0.88(2)$ & $2.05(2)$ & $2.8196(19)$ & $144.7(18)$ \\
$\mathrm{N} 1-\mathrm{H} 3 N \cdots \mathrm{O} 1 W$ & $0.92(2)$ & $1.87(3)$ & $2.7762(19)$ & $166(2)$ \\
$\mathrm{N} 3-\mathrm{H} 4 N \cdots \mathrm{N} 2^{\mathrm{iii}}$ & $0.89(2)$ & $2.02(2)$ & $2.9092(17)$ & $176.8(19)$ \\
$\mathrm{N} 3-\mathrm{H} 4 N \cdots \mathrm{O} 2^{i i i}$ & $0.89(2)$ & $2.58(2)$ & $3.1307(17)$ & $121.2(15)$ \\
$\mathrm{O} 1 W-\mathrm{H} 1 W \cdots \mathrm{F} 4^{\mathrm{iv}}$ & $0.87(1)$ & $2.03(1)$ & $2.8790(15)$ & $165(2)$ \\
$\mathrm{O} 1 W-\mathrm{H} 2 W \cdots \mathrm{F} 1$ & $0.88(1)$ & $1.94(1)$ & $2.7863(17)$ & $163(2)$ \\
\hline
\end{tabular}

Symmetry codes: (i) $-x+1 / 2, y+1 / 2,-z+3 / 2$; (ii) $-x,-y+1,-z+2$; (iii) $-x,-y,-z+2$; (iv) $x-1, y, z$.

\section{(VI) 2-\{[(4-Azaniumylphenyl)sulfonyl]azanidyl\}pyrimidin-1-ium nitrate}

\section{Crystal data}

$\mathrm{C}_{10} \mathrm{H}_{11} \mathrm{~N}_{4} \mathrm{O}_{2} \mathrm{~S}^{+} \cdot \mathrm{NO}_{3}^{-}$

$M_{r}=313.30$

Triclinic, $P \overline{1}$

Hall symbol: -P 1

$a=5.4705(4) \AA$

$b=10.1235(7) \AA$

$c=11.9956(8) \AA$

$\alpha=86.708(6)^{\circ}$

$\beta=85.489(6)^{\circ}$

$\gamma=74.394(7)^{\circ}$

$V=637.40(8) \AA^{3}$

\section{Data collection}

Oxford Diffraction Xcalibur E diffractometer

Radiation source: fine-focus sealed tube Graphite monochromator $\omega$ scans
$Z=2$

$F(000)=324$

$D_{\mathrm{x}}=1.632 \mathrm{Mg} \mathrm{m}^{-3}$

Mo $K \alpha$ radiation, $\lambda=0.71073 \AA$

Cell parameters from 3307 reflections

$\theta=3.4-29.4^{\circ}$

$\mu=0.29 \mathrm{~mm}^{-1}$

$T=123 \mathrm{~K}$

Fragment, light yellow

$0.26 \times 0.15 \times 0.06 \mathrm{~mm}$

Absorption correction: multi-scan

(CrysAlis PRO; Oxford Diffraction, 2010)

$T_{\min }=0.897, T_{\max }=1.000$

7390 measured reflections

2977 independent reflections

2429 reflections with $I>2 \sigma(I)$ 


$\begin{array}{lc}R_{\text {int }}=0.026 & k=-13 \rightarrow 13 \\ \theta_{\max }=28.0^{\circ}, \theta_{\min }=3.4^{\circ} & l=-15 \rightarrow 15 \\ h=-6 \rightarrow 7 & \\ & \\ \text { Refinement } & \text { Secondary atom site location: difference Fourier } \\ \text { Refinement on } F^{2} & \text { map } \\ \text { Least-squares matrix: full } & \text { Hydrogen site location: inferred from } \\ R\left[F^{2}>2 \sigma\left(F^{2}\right)\right]=0.045 & \text { neighbouring sites } \\ w R\left(F^{2}\right)=0.110 & \text { H atoms treated by a mixture of independent } \\ S=1.04 & \text { and constrained refinement } \\ 2977 \text { reflections } & w=1 /\left[\sigma^{2}\left(F_{\mathrm{o}}^{2}\right)+(0.0489 P)^{2}+0.4298 P\right] \\ 206 \text { parameters } & \text { where } P=\left(F_{\mathrm{o}}^{2}+2 F_{\mathrm{c}}^{2}\right) / 3 \\ 0 \text { restraints } & (\Delta / \sigma)_{\max }<0.001 \\ \text { Primary atom site location: structure-invariant } & \Delta \rho_{\max }=0.78 \text { e } \AA^{-3} \\ \quad \text { direct methods } & \Delta \rho_{\min }=-0.38 \text { e } \AA^{-3}\end{array}$

Special details

Experimental. Absorption correction: CrysAlisPro, Oxford Diffraction Ltd., Version 1.171.34.40 (release 27-08-2010 CrysAlis171 .NET) (compiled Aug 27 2010,11:50:40) Empirical absorption correction using spherical harmonics, implemented in SCALE3 ABSPACK scaling algorithm.

Geometry. All s.u.'s (except the s.u. in the dihedral angle between two l.s. planes) are estimated using the full covariance matrix. The cell s.u.'s are taken into account individually in the estimation of s.u.'s in distances, angles and torsion angles; correlations between s.u.'s in cell parameters are only used when they are defined by crystal symmetry. An approximate (isotropic) treatment of cell s.u.'s is used for estimating s.u.'s involving l.s. planes.

Refinement. Refinement of $F^{2}$ against ALL reflections. The weighted $R$-factor $w R$ and goodness of fit $S$ are based on $F^{2}$, conventional $R$-factors $R$ are based on $F$, with $F$ set to zero for negative $F^{2}$. The threshold expression of $F^{2}>2 \sigma\left(F^{2}\right)$ is used only for calculating $R$-factors (gt) etc. and is not relevant to the choice of reflections for refinement. $R$-factors based on $F^{2}$ are statistically about twice as large as those based on $F$, and $R$ - factors based on ALL data will be even larger.

Fractional atomic coordinates and isotropic or equivalent isotropic displacement parameters $\left(\AA^{2}\right)$

\begin{tabular}{lllll}
\hline & $x$ & $y$ & $z$ & $U_{\text {iso }} * / U_{\text {eq }}$ \\
\hline S1 & $0.31611(10)$ & $-0.07161(5)$ & $0.77390(4)$ & $0.01458(14)$ \\
O1 & $0.5490(3)$ & $-0.18213(14)$ & $0.76593(12)$ & $0.0182(3)$ \\
O2 & $0.0876(3)$ & $-0.10972(16)$ & $0.75678(12)$ & $0.0215(3)$ \\
O3 & $0.0278(3)$ & $0.5197(2)$ & $0.26381(18)$ & $0.0504(6)$ \\
O4 & $-0.3521(3)$ & $0.50354(18)$ & $0.24688(14)$ & $0.0311(4)$ \\
O5 & $-0.1819(3)$ & $0.45362(15)$ & $0.40662(12)$ & $0.0207(3)$ \\
N1 & $0.3888(4)$ & $0.35138(19)$ & $0.41482(15)$ & $0.0169(4)$ \\
N2 & $0.3246(3)$ & $-0.00844(18)$ & $0.89180(14)$ & $0.0159(4)$ \\
N3 & $0.2015(3)$ & $0.16323(19)$ & $1.01576(14)$ & $0.0166(4)$ \\
N4 & $-0.0507(3)$ & $0.17178(19)$ & $0.86457(14)$ & $0.0187(4)$ \\
N5 & $-0.1690(4)$ & $0.49396(19)$ & $0.30339(16)$ & $0.0222(4)$ \\
C1 & $0.3766(4)$ & $0.2491(2)$ & $0.50395(16)$ & $0.0147(4)$ \\
C2 & $0.2027(4)$ & $0.1732(2)$ & $0.49778(17)$ & $0.0159(4)$ \\
H2 & 0.0979 & 0.1869 & 0.4364 & $0.019 *$ \\
C3 & $0.1839(4)$ & $0.0768(2)$ & $0.58262(16)$ & $0.0154(4)$ \\
H3 & 0.0623 & 0.0255 & 0.5810 & $0.018 *$ \\
C4 & $0.3440(4)$ & $0.0559(2)$ & $0.66987(16)$ & $0.0132(4)$ \\
C5 & $0.5219(4)$ & $0.1303(2)$ & $0.67457(17)$ & $0.0158(4)$ \\
& & & &
\end{tabular}




$\begin{array}{lllll}\text { H5 } & 0.6323 & 0.1139 & 0.7341 & 0.019^{*} \\ \text { C6 } & 0.5361(4) & 0.2292(2) & 0.59076(17) & 0.0160(4) \\ \text { H6 } & 0.6542 & 0.2825 & 0.5931 & 0.019^{*} \\ \text { C7 } & 0.1540(4) & 0.1084(2) & 0.92134(16) & 0.0154(4) \\ \text { C8 } & 0.0485(4) & 0.2791(2) & 1.05659(17) & 0.0192(4) \\ \text { H8 } & 0.0846 & 0.3139 & 1.1235 & 0.023^{*} \\ \text { C9 } & -0.1597(4) & 0.3466(2) & 1.00104(19) & 0.0227(5) \\ \text { H9 } & -0.2724 & 0.4293 & 1.0273 & 0.027^{*} \\ \text { C10 } & -0.1989(4) & 0.2886(2) & 0.90410(19) & 0.0229(5) \\ \text { H10 } & -0.3410 & 0.3354 & 0.8634 & 0.027^{*} \\ \text { H1N } & 0.528(6) & 0.392(3) & 0.419(2) & 0.041(8)^{*} \\ \text { H2N } & 0.253(6) & 0.417(3) & 0.415(2) & 0.041(9)^{*} \\ \text { H3N } & 0.408(5) & 0.307(3) & 0.346(2) & 0.035(8)^{*} \\ \text { H4N } & 0.336(5) & 0.119(3) & 1.046(2) & 0.026(7)^{*}\end{array}$

Atomic displacement parameters $\left(\AA^{2}\right)$

\begin{tabular}{lllllll}
\hline & $U^{11}$ & $U^{22}$ & $U^{33}$ & $U^{12}$ & $U^{13}$ & $U^{23}$ \\
\hline S1 & $0.0165(3)$ & $0.0174(3)$ & $0.0102(2)$ & $-0.00517(19)$ & $-0.00247(18)$ & $0.00136(18)$ \\
O1 & $0.0222(8)$ & $0.0169(7)$ & $0.0139(7)$ & $-0.0022(6)$ & $-0.0027(6)$ & $0.0002(6)$ \\
O2 & $0.0239(9)$ & $0.0264(8)$ & $0.0180(8)$ & $-0.0135(7)$ & $-0.0056(6)$ & $0.0061(6)$ \\
O3 & $0.0225(10)$ & $0.0633(14)$ & $0.0593(14)$ & $-0.0107(9)$ & $0.0046(9)$ & $0.0348(11)$ \\
O4 & $0.0343(10)$ & $0.0356(10)$ & $0.0215(9)$ & $-0.0050(8)$ & $-0.0109(7)$ & $0.0052(7)$ \\
O5 & $0.0255(9)$ & $0.0217(8)$ & $0.0147(7)$ & $-0.0060(6)$ & $-0.0035(6)$ & $0.0020(6)$ \\
N1 & $0.0210(10)$ & $0.0149(9)$ & $0.0139(9)$ & $-0.0034(8)$ & $-0.0004(7)$ & $0.0008(7)$ \\
N2 & $0.0170(9)$ & $0.0200(9)$ & $0.0093(8)$ & $-0.0020(7)$ & $-0.0033(6)$ & $0.0007(7)$ \\
N3 & $0.0158(9)$ & $0.0205(9)$ & $0.0117(8)$ & $-0.0018(7)$ & $-0.0023(7)$ & $0.0026(7)$ \\
N4 & $0.0166(9)$ & $0.0235(9)$ & $0.0136(8)$ & $-0.0012(7)$ & $-0.0032(7)$ & $0.0025(7)$ \\
N5 & $0.0188(10)$ & $0.0213(9)$ & $0.0223(10)$ & $0.0003(8)$ & $0.0016(8)$ & $0.0040(8)$ \\
C1 & $0.0160(10)$ & $0.0138(9)$ & $0.0114(9)$ & $0.0001(8)$ & $0.0019(8)$ & $0.0003(7)$ \\
C2 & $0.0163(11)$ & $0.0191(10)$ & $0.0122(9)$ & $-0.0044(8)$ & $-0.0033(8)$ & $0.0017(8)$ \\
C3 & $0.0135(10)$ & $0.0200(10)$ & $0.0138(10)$ & $-0.0067(8)$ & $-0.0007(8)$ & $0.0001(8)$ \\
C4 & $0.0121(10)$ & $0.0145(9)$ & $0.0115(9)$ & $-0.0012(7)$ & $-0.0002(7)$ & $0.0002(7)$ \\
C5 & $0.0138(10)$ & $0.0194(10)$ & $0.0140(10)$ & $-0.0031(8)$ & $-0.0042(8)$ & $-0.0015(8)$ \\
C6 & $0.0146(10)$ & $0.0166(10)$ & $0.0176(10)$ & $-0.0050(8)$ & $-0.0004(8)$ & $-0.0021(8)$ \\
C7 & $0.0154(10)$ & $0.0205(10)$ & $0.0103(9)$ & $-0.0061(8)$ & $0.0007(8)$ & $0.0026(8)$ \\
C8 & $0.0240(12)$ & $0.0193(10)$ & $0.0135(10)$ & $-0.0050(9)$ & $0.0010(8)$ & $0.0001(8)$ \\
C9 & $0.0231(12)$ & $0.0202(11)$ & $0.0205(11)$ & $0.0011(9)$ & $-0.0001(9)$ & $0.0004(9)$ \\
C10 & $0.0192(11)$ & $0.0254(11)$ & $0.0204(11)$ & $-0.0005(9)$ & $-0.0025(9)$ & $0.0047(9)$ \\
& & & & & & \\
\hline
\end{tabular}

Geometric parameters $\left(\AA,{ }^{\circ}\right)$

\begin{tabular}{llll}
\hline $\mathrm{S} 1-\mathrm{O} 2$ & $1.4365(15)$ & $\mathrm{N} 4-\mathrm{C} 7$ & $1.346(3)$ \\
$\mathrm{S} 1-\mathrm{O} 1$ & $1.4527(15)$ & $\mathrm{C} 1-\mathrm{C} 2$ & $1.382(3)$ \\
$\mathrm{S} 1-\mathrm{N} 2$ & $1.5941(17)$ & $\mathrm{C} 1-\mathrm{C} 6$ & $1.382(3)$ \\
$\mathrm{S} 1-\mathrm{C} 4$ & $1.771(2)$ & $\mathrm{C} 2-\mathrm{C} 3$ & $1.386(3)$ \\
$\mathrm{O} 3-\mathrm{N} 5$ & $1.228(3)$ & $\mathrm{C} 2-\mathrm{H} 2$ & 0.9500 \\
$\mathrm{O} 4-\mathrm{N} 5$ & $1.233(2)$ & $\mathrm{C} 3-\mathrm{C} 4$ & $1.388(3)$
\end{tabular}




\begin{tabular}{|c|c|c|c|}
\hline $\mathrm{O} 5-\mathrm{N} 5$ & $1.284(2)$ & $\mathrm{C} 3-\mathrm{H} 3$ & 0.9500 \\
\hline $\mathrm{N} 1-\mathrm{C} 1$ & $1.455(3)$ & $\mathrm{C} 4-\mathrm{C} 5$ & $1.388(3)$ \\
\hline $\mathrm{N} 1-\mathrm{H} 1 \mathrm{~N}$ & $0.96(3)$ & $\mathrm{C} 5-\mathrm{C} 6$ & $1.390(3)$ \\
\hline $\mathrm{N} 1-\mathrm{H} 2 \mathrm{~N}$ & $0.85(3)$ & $\mathrm{C} 5-\mathrm{H} 5$ & 0.9500 \\
\hline $\mathrm{N} 1-\mathrm{H} 3 \mathrm{~N}$ & $0.95(3)$ & C6-H6 & 0.9500 \\
\hline $\mathrm{N} 2-\mathrm{C} 7$ & $1.341(3)$ & $\mathrm{C} 8-\mathrm{C} 9$ & $1.363(3)$ \\
\hline $\mathrm{N} 3-\mathrm{C} 8$ & $1.338(3)$ & $\mathrm{C} 8-\mathrm{H} 8$ & 0.9500 \\
\hline N3-C7 & $1.362(3)$ & $\mathrm{C} 9-\mathrm{C} 10$ & $1.389(3)$ \\
\hline $\mathrm{N} 3-\mathrm{H} 4 \mathrm{~N}$ & $0.85(3)$ & C9-H9 & 0.9500 \\
\hline $\mathrm{N} 4-\mathrm{C} 10$ & $1.330(3)$ & $\mathrm{C} 10-\mathrm{H} 10$ & 0.9500 \\
\hline $\mathrm{O} 2-\mathrm{S} 1-\mathrm{O} 1$ & $115.35(9)$ & $\mathrm{C} 3-\mathrm{C} 2-\mathrm{H} 2$ & 120.5 \\
\hline $\mathrm{O} 2-\mathrm{S} 1-\mathrm{N} 2$ & $115.72(9)$ & $\mathrm{C} 2-\mathrm{C} 3-\mathrm{C} 4$ & $119.55(19)$ \\
\hline $\mathrm{O} 1-\mathrm{S} 1-\mathrm{N} 2$ & $103.48(9)$ & $\mathrm{C} 2-\mathrm{C} 3-\mathrm{H} 3$ & 120.2 \\
\hline $\mathrm{O} 2-\mathrm{S} 1-\mathrm{C} 4$ & $107.13(9)$ & $\mathrm{C} 4-\mathrm{C} 3-\mathrm{H} 3$ & 120.2 \\
\hline $\mathrm{O} 1-\mathrm{S} 1-\mathrm{C} 4$ & $107.81(9)$ & $\mathrm{C} 3-\mathrm{C} 4-\mathrm{C} 5$ & $121.34(19)$ \\
\hline $\mathrm{N} 2-\mathrm{S} 1-\mathrm{C} 4$ & $106.87(9)$ & $\mathrm{C} 3-\mathrm{C} 4-\mathrm{S} 1$ & $117.43(15)$ \\
\hline $\mathrm{C} 1-\mathrm{N} 1-\mathrm{H} 1 \mathrm{~N}$ & 113.7 (17) & $\mathrm{C} 5-\mathrm{C} 4-\mathrm{S} 1$ & $121.22(15)$ \\
\hline $\mathrm{C} 1-\mathrm{N} 1-\mathrm{H} 2 \mathrm{~N}$ & $112(2)$ & $\mathrm{C} 4-\mathrm{C} 5-\mathrm{C} 6$ & $118.94(18)$ \\
\hline $\mathrm{H} 1 \mathrm{~N}-\mathrm{N} 1-\mathrm{H} 2 \mathrm{~N}$ & $107(3)$ & $\mathrm{C} 4-\mathrm{C} 5-\mathrm{H} 5$ & 120.5 \\
\hline $\mathrm{C} 1-\mathrm{N} 1-\mathrm{H} 3 \mathrm{~N}$ & $107.0(17)$ & $\mathrm{C} 6-\mathrm{C} 5-\mathrm{H} 5$ & 120.5 \\
\hline $\mathrm{H} 1 \mathrm{~N}-\mathrm{N} 1-\mathrm{H} 3 \mathrm{~N}$ & $109(2)$ & $\mathrm{C} 1-\mathrm{C} 6-\mathrm{C} 5$ & 119.34 (19) \\
\hline $\mathrm{H} 2 \mathrm{~N}-\mathrm{N} 1-\mathrm{H} 3 \mathrm{~N}$ & $109(2)$ & $\mathrm{C} 1-\mathrm{C} 6-\mathrm{H} 6$ & 120.3 \\
\hline $\mathrm{C} 7-\mathrm{N} 2-\mathrm{S} 1$ & $120.20(14)$ & $\mathrm{C} 5-\mathrm{C} 6-\mathrm{H} 6$ & 120.3 \\
\hline $\mathrm{C} 8-\mathrm{N} 3-\mathrm{C} 7$ & $122.28(19)$ & $\mathrm{N} 2-\mathrm{C} 7-\mathrm{N} 4$ & $124.84(19)$ \\
\hline $\mathrm{C} 8-\mathrm{N} 3-\mathrm{H} 4 \mathrm{~N}$ & $122.6(17)$ & $\mathrm{N} 2-\mathrm{C} 7-\mathrm{N} 3$ & $114.98(18)$ \\
\hline $\mathrm{C} 7-\mathrm{N} 3-\mathrm{H} 4 \mathrm{~N}$ & $115.1(17)$ & $\mathrm{N} 4-\mathrm{C} 7-\mathrm{N} 3$ & $120.18(19)$ \\
\hline $\mathrm{C} 10-\mathrm{N} 4-\mathrm{C} 7$ & $117.29(19)$ & $\mathrm{N} 3-\mathrm{C} 8-\mathrm{C} 9$ & $119.2(2)$ \\
\hline $\mathrm{O} 3-\mathrm{N} 5-\mathrm{O} 4$ & $122.2(2)$ & $\mathrm{N} 3-\mathrm{C} 8-\mathrm{H} 8$ & 120.4 \\
\hline $\mathrm{O} 3-\mathrm{N} 5-\mathrm{O} 5$ & $118.7(2)$ & $\mathrm{C} 9-\mathrm{C} 8-\mathrm{H} 8$ & 120.4 \\
\hline $\mathrm{O} 4-\mathrm{N} 5-\mathrm{O} 5$ & $119.15(19)$ & $\mathrm{C} 8-\mathrm{C} 9-\mathrm{C} 10$ & $116.7(2)$ \\
\hline $\mathrm{C} 2-\mathrm{C} 1-\mathrm{C} 6$ & $121.87(19)$ & $\mathrm{C} 8-\mathrm{C} 9-\mathrm{H} 9$ & 121.7 \\
\hline $\mathrm{C} 2-\mathrm{C} 1-\mathrm{N} 1$ & $117.73(18)$ & $\mathrm{C} 10-\mathrm{C} 9-\mathrm{H} 9$ & 121.7 \\
\hline $\mathrm{C} 6-\mathrm{C} 1-\mathrm{N} 1$ & $120.40(19)$ & $\mathrm{N} 4-\mathrm{C} 10-\mathrm{C} 9$ & $124.3(2)$ \\
\hline $\mathrm{C} 1-\mathrm{C} 2-\mathrm{C} 3$ & $118.92(18)$ & $\mathrm{N} 4-\mathrm{C} 10-\mathrm{H} 10$ & 117.8 \\
\hline $\mathrm{C} 1-\mathrm{C} 2-\mathrm{H} 2$ & 120.5 & $\mathrm{C} 9-\mathrm{C} 10-\mathrm{H} 10$ & 117.8 \\
\hline $\mathrm{O} 2-\mathrm{S} 1-\mathrm{N} 2-\mathrm{C} 7$ & $-61.49(19)$ & $\mathrm{S} 1-\mathrm{C} 4-\mathrm{C} 5-\mathrm{C} 6$ & $179.99(15)$ \\
\hline $\mathrm{O} 1-\mathrm{S} 1-\mathrm{N} 2-\mathrm{C} 7$ & $171.35(16)$ & $\mathrm{C} 2-\mathrm{C} 1-\mathrm{C} 6-\mathrm{C} 5$ & $-0.1(3)$ \\
\hline $\mathrm{C} 4-\mathrm{S} 1-\mathrm{N} 2-\mathrm{C} 7$ & $57.69(18)$ & $\mathrm{N} 1-\mathrm{C} 1-\mathrm{C} 6-\mathrm{C} 5$ & $-179.59(18)$ \\
\hline $\mathrm{C} 6-\mathrm{C} 1-\mathrm{C} 2-\mathrm{C} 3$ & $1.6(3)$ & $\mathrm{C} 4-\mathrm{C} 5-\mathrm{C} 6-\mathrm{C} 1$ & $-1.2(3)$ \\
\hline $\mathrm{N} 1-\mathrm{C} 1-\mathrm{C} 2-\mathrm{C} 3$ & $-178.85(18)$ & $\mathrm{S} 1-\mathrm{N} 2-\mathrm{C} 7-\mathrm{N} 4$ & $8.6(3)$ \\
\hline $\mathrm{C} 1-\mathrm{C} 2-\mathrm{C} 3-\mathrm{C} 4$ & $-1.8(3)$ & $\mathrm{S} 1-\mathrm{N} 2-\mathrm{C} 7-\mathrm{N} 3$ & $-170.79(14)$ \\
\hline $\mathrm{C} 2-\mathrm{C} 3-\mathrm{C} 4-\mathrm{C} 5$ & $0.5(3)$ & $\mathrm{C} 10-\mathrm{N} 4-\mathrm{C} 7-\mathrm{N} 2$ & $-178.8(2)$ \\
\hline $\mathrm{C} 2-\mathrm{C} 3-\mathrm{C} 4-\mathrm{S} 1$ & $-178.48(15)$ & $\mathrm{C} 10-\mathrm{N} 4-\mathrm{C} 7-\mathrm{N} 3$ & $0.6(3)$ \\
\hline $\mathrm{O} 2-\mathrm{S} 1-\mathrm{C} 4-\mathrm{C} 3$ & $-11.74(19)$ & $\mathrm{C} 8-\mathrm{N} 3-\mathrm{C} 7-\mathrm{N} 2$ & $-179.64(19)$ \\
\hline $\mathrm{O} 1-\mathrm{S} 1-\mathrm{C} 4-\mathrm{C} 3$ & $112.98(16)$ & $\mathrm{C} 8-\mathrm{N} 3-\mathrm{C} 7-\mathrm{N} 4$ & $1.0(3)$ \\
\hline $\mathrm{N} 2-\mathrm{S} 1-\mathrm{C} 4-\mathrm{C} 3$ & $-136.34(16)$ & $\mathrm{C} 7-\mathrm{N} 3-\mathrm{C} 8-\mathrm{C} 9$ & $-1.3(3)$ \\
\hline
\end{tabular}




$\begin{array}{llll}\mathrm{O} 2-\mathrm{S} 1-\mathrm{C} 4-\mathrm{C} 5 & 169.27(16) & \mathrm{N} 3-\mathrm{C} 8-\mathrm{C} 9-\mathrm{C} 10 & 0.2(3) \\ \mathrm{O} 1-\mathrm{S} 1-\mathrm{C} 4-\mathrm{C} 5 & -66.01(18) & \mathrm{C} 7-\mathrm{N} 4-\mathrm{C} 10-\mathrm{C} 9 & -1.8(3) \\ \mathrm{N} 2-\mathrm{S} 1-\mathrm{C} 4-\mathrm{C} 5 & 44.67(19) & \mathrm{C} 8-\mathrm{C} 9-\mathrm{C} 10-\mathrm{N} 4 & 1.4(4) \\ \mathrm{C} 3-\mathrm{C} 4-\mathrm{C} 5-\mathrm{C} 6 & 1.0(3) & & \end{array}$

Hydrogen-bond geometry $\left(\AA,{ }^{\circ}\right)$

\begin{tabular}{lllll}
\hline$D-\mathrm{H} \cdots A$ & $D-\mathrm{H}$ & $\mathrm{H} \cdots A$ & $D \cdots A$ & $D-\mathrm{H} \cdots A$ \\
\hline $\mathrm{N} 1-\mathrm{H} 1 N \cdots \mathrm{O} 5^{\mathrm{i}}$ & $0.96(3)$ & $1.85(3)$ & $2.803(3)$ & $171(3)$ \\
$\mathrm{N} 1-\mathrm{H} 1 N \cdots 4^{\mathrm{i}}$ & $0.96(3)$ & $2.42(3)$ & $2.968(3)$ & $116(2)$ \\
$\mathrm{N} 1-\mathrm{H} 2 N \cdots \mathrm{O} 3$ & $0.85(3)$ & $2.31(3)$ & $2.912(3)$ & $128(3)$ \\
$\mathrm{N} 1-\mathrm{H} 2 N \cdots \mathrm{O} 5$ & $0.85(3)$ & $2.32(3)$ & $3.021(3)$ & $140(3)$ \\
$\mathrm{N} 1-\mathrm{H} 3 N \cdots \mathrm{O} 1^{i i}$ & $0.95(3)$ & $1.85(3)$ & $2.784(2)$ & $166(3)$ \\
$\mathrm{N} 3-\mathrm{H} 4 N \cdots \mathrm{N} 2^{2 i i}$ & $0.85(3)$ & $2.06(3)$ & $2.911(3)$ & $176(2)$ \\
$\mathrm{N} 3-\mathrm{H} 4 N \cdots \mathrm{O} 1^{i i i}$ & $0.85(3)$ & $2.55(3)$ & $3.085(2)$ & $122(2)$ \\
\hline
\end{tabular}

Symmetry codes: (i) $x+1, y, z$; (ii) $-x+1,-y,-z+1$; (iii) $-x+1,-y,-z+2$.

\section{(VII) 4-[(Pyrimidin-2-yl)sulfamoyl]anilinium ethanesulfonate}

\section{Crystal data}

$\mathrm{C}_{10} \mathrm{H}_{11} \mathrm{~N}_{4} \mathrm{O}_{2} \mathrm{~S}^{+} \cdot \mathrm{C}_{2} \mathrm{H}_{5} \mathrm{O}_{3} \mathrm{~S}^{-}$

$M_{r}=360.41$

Monoclinic, $P 2_{1} / c$

Hall symbol: -P $2 \mathrm{ybc}$

$a=5.5269(4) \AA$

$b=34.979(3) \AA$

$c=8.4395(6) \AA$

$\beta=93.102(7)^{\circ}$

$V=1629.2(2) \AA^{3}$

$Z=4$

\section{Data collection}

Oxford Diffraction Xcalibur E diffractometer

Radiation source: fine-focus sealed tube

Graphite monochromator

$\omega$ scans

Absorption correction: multi-scan

(CrysAlis PRO; Oxford Diffraction, 2010)

$T_{\min }=0.908, T_{\max }=1.000$

\section{Refinement}

Refinement on $F^{2}$

Least-squares matrix: full

$R\left[F^{2}>2 \sigma\left(F^{2}\right)\right]=0.055$

$w R\left(F^{2}\right)=0.112$

$S=1.01$

3515 reflections

222 parameters

3 restraints

Primary atom site location: structure-invariant direct methods
$F(000)=752$

$D_{\mathrm{x}}=1.469 \mathrm{Mg} \mathrm{m}^{-3}$

Mo $K \alpha$ radiation, $\lambda=0.71073 \AA$

Cell parameters from 2991 reflections

$\theta=3.4-28.7^{\circ}$

$\mu=0.36 \mathrm{~mm}^{-1}$

$T=123 \mathrm{~K}$

Needle, colourless

$0.4 \times 0.03 \times 0.02 \mathrm{~mm}$

19991 measured reflections

3515 independent reflections

2212 reflections with $I>2 \sigma(I)$

$R_{\text {int }}=0.104$

$\theta_{\max }=27.0^{\circ}, \theta_{\min }=3.4^{\circ}$

$h=-7 \rightarrow 7$

$k=-43 \rightarrow 44$

$l=-10 \rightarrow 10$

Secondary atom site location: difference Fourier map

Hydrogen site location: inferred from neighbouring sites

$\mathrm{H}$ atoms treated by a mixture of independent and constrained refinement

$w=1 /\left[\sigma^{2}\left(F_{\mathrm{o}}^{2}\right)+(0.0391 P)^{2}+0.6311 P\right]$

where $P=\left(F_{\mathrm{o}}^{2}+2 F_{\mathrm{c}}^{2}\right) / 3$

$(\Delta / \sigma)_{\max }=0.001$

$\Delta \rho_{\max }=0.35 \mathrm{e} \AA^{-3}$

$\Delta \rho_{\min }=-0.41$ e $\AA^{-3}$ 


\section{Special details}

Experimental. Absorption correction: CrysAlisPro, Oxford Diffraction Ltd., Version 1.171.34.40 (release 27-08-2010 CrysAlis171 .NET) (compiled Aug 27 2010,11:50:40) Empirical absorption correction using spherical harmonics, implemented in SCALE3 ABSPACK scaling algorithm.

Geometry. All s.u.'s (except the s.u. in the dihedral angle between two 1.s. planes) are estimated using the full covariance matrix. The cell s.u.'s are taken into account individually in the estimation of s.u.'s in distances, angles and torsion angles; correlations between s.u.'s in cell parameters are only used when they are defined by crystal symmetry. An approximate (isotropic) treatment of cell s.u.'s is used for estimating s.u.'s involving 1.s. planes.

Refinement. Refinement of $\mathrm{F}^{2}$ against ALL reflections. The weighted R-factor $\mathrm{wR}$ and goodness of fit $\mathrm{S}$ are based on $\mathrm{F}^{2}$, conventional R-factors $\mathrm{R}$ are based on $\mathrm{F}$, with $\mathrm{F}$ set to zero for negative $\mathrm{F}^{2}$. The threshold expression of $\mathrm{F}^{2}>2 \sigma\left(\mathrm{F}^{2}\right)$ is used only for calculating R-factors(gt) etc. and is not relevant to the choice of reflections for refinement. R-factors based on $\mathrm{F}^{2}$ are statistically about twice as large as those based on F, and R- factors based on ALL data will be even larger.

Fractional atomic coordinates and isotropic or equivalent isotropic displacement parameters $\left(\AA^{2}\right)$

\begin{tabular}{|c|c|c|c|c|}
\hline & $x$ & $y$ & $z$ & $U_{\text {iso }} * / U_{\text {eq }}$ \\
\hline S1 & $0.28192(13)$ & $0.44093(2)$ & $0.77910(9)$ & $0.0177(2)$ \\
\hline S2 & $0.00973(13)$ & $0.27930(2)$ & $0.23275(9)$ & $0.0181(2)$ \\
\hline $\mathrm{O} 1$ & $0.4676(4)$ & $0.45420(6)$ & $0.8902(2)$ & $0.0224(5)$ \\
\hline $\mathrm{O} 2$ & $0.0371(4)$ & $0.44002(6)$ & $0.8241(2)$ & $0.0213(5)$ \\
\hline $\mathrm{O} 3$ & -0.1498 & $0.24673(6)$ & $0.2104(3)$ & $0.0282(6)$ \\
\hline $\mathrm{O} 4$ & $-0.0356(4)$ & $0.30107(7)$ & $0.3739(3)$ & $0.0271(6)$ \\
\hline O5 & $0.2670(3)$ & $0.26823(6)$ & $0.2301(3)$ & $0.0222(5)$ \\
\hline N1 & $0.4972(5)$ & $0.28671(8)$ & 0.5241 (3) & $0.0215(6)$ \\
\hline $\mathrm{H} 1 \mathrm{~N}$ & $0.452(6)$ & $0.2671(7)$ & $0.586(3)$ & $0.032 *$ \\
\hline $\mathrm{H} 2 \mathrm{~N}$ & $0.656(2)$ & $0.2841(10)$ & $0.505(4)$ & $0.032 *$ \\
\hline $\mathrm{H} 3 \mathrm{~N}$ & $0.420(5)$ & $0.2818(9)$ & $0.428(2)$ & $0.032^{*}$ \\
\hline $\mathrm{N} 2$ & $0.3082(5)$ & $0.46787(8)$ & $0.6229(3)$ & $0.0184(6)$ \\
\hline N3 & $0.0015(5)$ & $0.43804(8)$ & $0.4667(3)$ & $0.0224(6)$ \\
\hline $\mathrm{N} 4$ & $0.2795(4)$ & $0.48159(7)$ & $0.3562(3)$ & $0.0184(6)$ \\
\hline $\mathrm{C} 1$ & $0.4512(5)$ & $0.32414(9)$ & $0.5930(4)$ & $0.0166(7)$ \\
\hline $\mathrm{C} 2$ & $0.2449(5)$ & $0.32892(9)$ & $0.6757(4)$ & $0.0189(7)$ \\
\hline $\mathrm{H} 2$ & 0.1388 & 0.3081 & 0.6919 & $0.023 *$ \\
\hline $\mathrm{C} 3$ & $0.1965(5)$ & $0.36487(9)$ & $0.7345(4)$ & $0.0180(7)$ \\
\hline $\mathrm{H} 3$ & 0.0538 & 0.3690 & 0.7901 & $0.022 *$ \\
\hline $\mathrm{C} 4$ & $0.3537(5)$ & $0.39464(9)$ & $0.7130(4)$ & $0.0155(7)$ \\
\hline $\mathrm{C} 5$ & $0.5653(5)$ & $0.38924(9)$ & $0.6337(4)$ & $0.0186(7)$ \\
\hline H5 & 0.6750 & 0.4098 & 0.6217 & $0.022 *$ \\
\hline C6 & $0.6134(5)$ & $0.35368(9)$ & $0.5727(4)$ & $0.0183(7)$ \\
\hline H6 & 0.7563 & 0.3495 & 0.5175 & $0.022 *$ \\
\hline $\mathrm{C} 7$ & $0.1892(5)$ & $0.46205(9)$ & $0.4759(4)$ & $0.0167(7)$ \\
\hline $\mathrm{C} 8$ & $-0.1070(6)$ & $0.43437(10)$ & $0.3223(4)$ & $0.0261(8)$ \\
\hline H8 & -0.2436 & 0.4179 & 0.3103 & $0.031 *$ \\
\hline C9 & $-0.0331(6)$ & $0.45291(9)$ & 0.1902 (4) & $0.0232(8)$ \\
\hline H9 & -0.1136 & 0.4497 & 0.0887 & $0.028 *$ \\
\hline $\mathrm{C} 10$ & $0.1660(6)$ & $0.47650(9)$ & $0.2140(4)$ & $0.0218(8)$ \\
\hline H10 & 0.2247 & 0.4897 & 0.1254 & $0.026^{*}$ \\
\hline C11 & $-0.0402(6)$ & $0.30986(10)$ & $0.0676(4)$ & $0.0294(9)$ \\
\hline H11A & 0.0793 & 0.3310 & 0.0752 & $0.035^{*}$ \\
\hline
\end{tabular}




\begin{tabular}{lllll} 
H11B & -0.0128 & 0.2953 & -0.0305 & $0.035^{*}$ \\
C12 & $-0.2942(6)$ & $0.32674(12)$ & $0.0553(5)$ & $0.0443(11)$ \\
H12A & -0.4136 & 0.3060 & 0.0450 & $0.066^{*}$ \\
H12B & -0.3112 & 0.3434 & -0.0380 & $0.066^{*}$ \\
H12C & -0.3215 & 0.3417 & 0.1510 & $0.066^{*}$ \\
H4N & $0.442(6)$ & $0.4822(10)$ & $0.627(4)$ & $0.031(10)^{*}$ \\
\hline
\end{tabular}

Atomic displacement parameters $\left(\AA^{2}\right)$

\begin{tabular}{|c|c|c|c|c|c|c|}
\hline & $U^{11}$ & $U^{22}$ & $U^{33}$ & $U^{12}$ & $U^{13}$ & $U^{23}$ \\
\hline $\mathrm{S} 1$ & $0.0194(4)$ & $0.0175(4)$ & $0.0167(4)$ & -0.0018 & $0.0050(3)$ & $-0.0007(4)$ \\
\hline $\mathrm{S} 2$ & $0.0156(4)$ & $0.0213(5)$ & $0.0172(4)$ & $-0.0003(3)$ & $0.0003(3)$ & -0.0022 (4) \\
\hline O1 & $0.0268(12)$ & $0.0252(13)$ & $0.0152(12)$ & $-0.0049(10)$ & $0.0015(9)$ & $-0.0029(10)$ \\
\hline $\mathrm{O} 2$ & $0.0216(11)$ & $0.0223(13)$ & $0.0208(13)$ & $0.0024(10)$ & $0.0084(9)$ & $-0.0009(10)$ \\
\hline $\mathrm{O} 3$ & $0.0204(12)$ & $0.0280(14)$ & $0.0354(15)$ & $-0.0057(10)$ & $-0.0078(10)$ & $0.0004(12)$ \\
\hline $\mathrm{O} 4$ & $0.0238(12)$ & $0.0355(15)$ & $0.0220(13)$ & $0.0042(11)$ & $0.0002(10)$ & $-0.0100(11)$ \\
\hline O5 & 0.0139 (11) & $0.0300(14)$ & $0.0228(13)$ & $0.0032(10)$ & $0.0025(9)$ & $-0.0052(10)$ \\
\hline N1 & $0.0216(15)$ & $0.0196(16)$ & $0.0230(17)$ & $0.0020(13)$ & $-0.0036(13)$ & $-0.0039(13)$ \\
\hline $\mathrm{N} 2$ & 0.0195 (14) & $0.0184(16)$ & $0.0176(15)$ & $-0.0054(13)$ & $0.0032(12)$ & $0.0020(12)$ \\
\hline N3 & $0.0243(15)$ & $0.0229(16)$ & $0.0202(16)$ & $-0.0062(13)$ & $0.0036(12)$ & $0.0001(13)$ \\
\hline N4 & $0.0216(14)$ & $0.0168(15)$ & $0.0173(15)$ & $0.0011(11)$ & $0.0064(11)$ & $0.0017(12)$ \\
\hline $\mathrm{C} 1$ & $0.0151(16)$ & $0.0192(18)$ & $0.0150(17)$ & 0.0037 (13) & $-0.0051(13)$ & $-0.0029(14)$ \\
\hline $\mathrm{C} 2$ & $0.0184(17)$ & $0.0160(18)$ & $0.0219(18)$ & $-0.0018(14)$ & $-0.0027(14)$ & $0.0019(15)$ \\
\hline $\mathrm{C} 3$ & 0.0163 (16) & 0.0204 (18) & $0.0179(18)$ & $-0.0006(14)$ & 0.0049 (13) & $0.0028(15)$ \\
\hline $\mathrm{C} 4$ & $0.0164(16)$ & $0.0158(17)$ & $0.0141(17)$ & $-0.0006(13)$ & -0.0009 & $0.0011(14)$ \\
\hline $\mathrm{C} 5$ & $0.0172(16)$ & $0.0197(18)$ & 0.0189 (18) & $-0.0050(14)$ & 0.0017 (13) & $0.0012(15)$ \\
\hline C6 & $0.0135(16)$ & $0.0231(19)$ & $0.0186(18)$ & $0.0003(14)$ & $0.0040(13)$ & $-0.0029(15)$ \\
\hline $\mathrm{C} 7$ & $0.0181(16)$ & $0.0156(18)$ & $0.0168(18)$ & 0.0037 (13) & $0.0055(13)$ & $-0.0021(14)$ \\
\hline $\mathrm{C} 8$ & $0.0251(18)$ & $0.027(2)$ & $0.026(2)$ & $-0.0061(15)$ & $0.0014(15)$ & $-0.0049(16)$ \\
\hline $\mathrm{C} 9$ & $0.0280(18)$ & $0.026(2)$ & $0.0154(18)$ & $-0.0035(15)$ & $0.0004(14)$ & $-0.0002(15)$ \\
\hline $\mathrm{C} 10$ & 0.0316 (19) & 0.0203 (19) & $0.0141(18)$ & $0.0043(15)$ & $0.0071(14)$ & $0.0009(15)$ \\
\hline $\mathrm{C} 11$ & 0.0266 (19) & $0.035(2)$ & $0.026(2)$ & $-0.0025(16)$ & $0.0045(15)$ & $0.0090(17)$ \\
\hline $\mathrm{C} 12$ & $0.034(2)$ & $0.054(3)$ & $0.045(3)$ & 0.0042 (19) & -0.0019 (19) & $0.021(2)$ \\
\hline
\end{tabular}

Geometric parameters $\left(A,{ }^{\circ}\right)$

\begin{tabular}{llll}
\hline $\mathrm{S} 1-\mathrm{O} 2$ & $1.425(2)$ & $\mathrm{C} 2-\mathrm{C} 3$ & $1.383(4)$ \\
$\mathrm{S} 1-\mathrm{O} 1$ & $1.430(2)$ & $\mathrm{C} 2-\mathrm{H} 2$ & 0.9500 \\
$\mathrm{~S} 1-\mathrm{N} 2$ & $1.633(3)$ & $\mathrm{C} 3-\mathrm{C} 4$ & $1.375(4)$ \\
$\mathrm{S} 1-\mathrm{C} 4$ & $1.765(3)$ & $\mathrm{C} 3-\mathrm{H} 3$ & 0.9500 \\
$\mathrm{~S} 2-\mathrm{O} 3$ & $1.447(2)$ & $\mathrm{C} 4-\mathrm{C} 5$ & $1.391(4)$ \\
$\mathrm{S} 2-\mathrm{O} 4$ & $1.447(2)$ & $\mathrm{C} 5-\mathrm{C} 6$ & $1.377(4)$ \\
$\mathrm{S} 2-\mathrm{O} 5$ & $1.475(2)$ & $\mathrm{C} 5-\mathrm{H} 5$ & 0.9500 \\
$\mathrm{~S} 2-\mathrm{C} 11$ & $1.766(3)$ & $\mathrm{C} 6-\mathrm{H} 6$ & 0.9500 \\
$\mathrm{~N} 1-\mathrm{C} 1$ & $1.460(4)$ & $\mathrm{C} 8-\mathrm{C} 9$ & $1.371(4)$ \\
$\mathrm{N} 1-\mathrm{H} 1 \mathrm{~N}$ & $0.905(10)$ & $\mathrm{C} 8-\mathrm{H} 8$ & 0.9500 \\
$\mathrm{~N} 1-\mathrm{H} 2 \mathrm{~N}$ & $0.902(10)$ & $\mathrm{C} 9-\mathrm{C} 10$ & $1.381(4)$ \\
$\mathrm{N} 1-\mathrm{H} 3 \mathrm{~N}$ & $0.913(10)$ & $\mathrm{C} 9-\mathrm{H} 9$ & 0.9500
\end{tabular}




\begin{tabular}{|c|c|c|c|}
\hline $\mathrm{N} 2-\mathrm{C} 7$ & $1.388(4)$ & $\mathrm{C} 10-\mathrm{H} 10$ & 0.9500 \\
\hline $\mathrm{N} 2-\mathrm{H} 4 \mathrm{~N}$ & $0.89(3)$ & $\mathrm{C} 11-\mathrm{C} 12$ & $1.521(5)$ \\
\hline $\mathrm{N} 3-\mathrm{C} 7$ & $1.334(4)$ & $\mathrm{C} 11-\mathrm{H} 11 \mathrm{~A}$ & 0.9900 \\
\hline $\mathrm{N} 3-\mathrm{C} 8$ & $1.335(4)$ & C11-H11B & 0.9900 \\
\hline $\mathrm{N} 4-\mathrm{C} 10$ & $1.336(4)$ & $\mathrm{C} 12-\mathrm{H} 12 \mathrm{~A}$ & 0.9800 \\
\hline $\mathrm{N} 4-\mathrm{C} 7$ & $1.338(4)$ & $\mathrm{C} 12-\mathrm{H} 12 \mathrm{~B}$ & 0.9800 \\
\hline $\mathrm{C} 1-\mathrm{C} 2$ & $1.379(4)$ & $\mathrm{C} 12-\mathrm{H} 12 \mathrm{C}$ & 0.9800 \\
\hline $\mathrm{C} 1-\mathrm{C} 6$ & $1.385(4)$ & & \\
\hline $\mathrm{O} 2-\mathrm{S} 1-\mathrm{O} 1$ & $119.39(13)$ & $\mathrm{C} 3-\mathrm{C} 4-\mathrm{S} 1$ & $119.9(2)$ \\
\hline $\mathrm{O} 2-\mathrm{S} 1-\mathrm{N} 2$ & $110.80(13)$ & $\mathrm{C} 5-\mathrm{C} 4-\mathrm{S} 1$ & $119.1(2)$ \\
\hline $\mathrm{O} 1-\mathrm{S} 1-\mathrm{N} 2$ & $104.48(14)$ & $\mathrm{C} 6-\mathrm{C} 5-\mathrm{C} 4$ & $119.1(3)$ \\
\hline $\mathrm{O} 2-\mathrm{S} 1-\mathrm{C} 4$ & $107.25(14)$ & $\mathrm{C} 6-\mathrm{C} 5-\mathrm{H} 5$ & 120.4 \\
\hline $\mathrm{O} 1-\mathrm{S} 1-\mathrm{C} 4$ & $109.85(13)$ & $\mathrm{C} 4-\mathrm{C} 5-\mathrm{H} 5$ & 120.4 \\
\hline $\mathrm{N} 2-\mathrm{S} 1-\mathrm{C} 4$ & $104.06(14)$ & $\mathrm{C} 5-\mathrm{C} 6-\mathrm{C} 1$ & $119.2(3)$ \\
\hline $\mathrm{O} 3-\mathrm{S} 2-\mathrm{O} 4$ & $112.99(14)$ & $\mathrm{C} 5-\mathrm{C} 6-\mathrm{H} 6$ & 120.4 \\
\hline $\mathrm{O} 3-\mathrm{S} 2-\mathrm{O} 5$ & $111.87(13)$ & $\mathrm{C} 1-\mathrm{C} 6-\mathrm{H} 6$ & 120.4 \\
\hline $\mathrm{O} 4-\mathrm{S} 2-\mathrm{O} 5$ & $111.13(13)$ & $\mathrm{N} 3-\mathrm{C} 7-\mathrm{N} 4$ & $126.8(3)$ \\
\hline $\mathrm{O} 3-\mathrm{S} 2-\mathrm{C} 11$ & $107.77(15)$ & $\mathrm{N} 3-\mathrm{C} 7-\mathrm{N} 2$ & $118.3(3)$ \\
\hline $\mathrm{O} 4-\mathrm{S} 2-\mathrm{C} 11$ & $107.73(16)$ & $\mathrm{N} 4-\mathrm{C} 7-\mathrm{N} 2$ & $115.0(3)$ \\
\hline $\mathrm{O} 5-\mathrm{S} 2-\mathrm{C} 11$ & $104.87(14)$ & $\mathrm{N} 3-\mathrm{C} 8-\mathrm{C} 9$ & $123.9(3)$ \\
\hline $\mathrm{C} 1-\mathrm{N} 1-\mathrm{H} 1 \mathrm{~N}$ & $113(2)$ & $\mathrm{N} 3-\mathrm{C} 8-\mathrm{H} 8$ & 118.0 \\
\hline $\mathrm{C} 1-\mathrm{N} 1-\mathrm{H} 2 \mathrm{~N}$ & $111(2)$ & $\mathrm{C} 9-\mathrm{C} 8-\mathrm{H} 8$ & 118.0 \\
\hline $\mathrm{H} 1 \mathrm{~N}-\mathrm{N} 1-\mathrm{H} 2 \mathrm{~N}$ & $109(3)$ & $\mathrm{C} 8-\mathrm{C} 9-\mathrm{C} 10$ & $115.8(3)$ \\
\hline $\mathrm{C} 1-\mathrm{N} 1-\mathrm{H} 3 \mathrm{~N}$ & $116(2)$ & $\mathrm{C} 8-\mathrm{C} 9-\mathrm{H} 9$ & 122.1 \\
\hline $\mathrm{H} 1 \mathrm{~N}-\mathrm{N} 1-\mathrm{H} 3 \mathrm{~N}$ & $104(3)$ & $\mathrm{C} 10-\mathrm{C} 9-\mathrm{H} 9$ & 122.1 \\
\hline $\mathrm{H} 2 \mathrm{~N}-\mathrm{N} 1-\mathrm{H} 3 \mathrm{~N}$ & $104(3)$ & $\mathrm{N} 4-\mathrm{C} 10-\mathrm{C} 9$ & $122.8(3)$ \\
\hline $\mathrm{C} 7-\mathrm{N} 2-\mathrm{S} 1$ & $125.4(2)$ & $\mathrm{N} 4-\mathrm{C} 10-\mathrm{H} 10$ & 118.6 \\
\hline $\mathrm{C} 7-\mathrm{N} 2-\mathrm{H} 4 \mathrm{~N}$ & $118(2)$ & $\mathrm{C} 9-\mathrm{C} 10-\mathrm{H} 10$ & 118.6 \\
\hline $\mathrm{S} 1-\mathrm{N} 2-\mathrm{H} 4 \mathrm{~N}$ & $113(2)$ & $\mathrm{C} 12-\mathrm{C} 11-\mathrm{S} 2$ & $113.1(2)$ \\
\hline $\mathrm{C} 7-\mathrm{N} 3-\mathrm{C} 8$ & $115.0(3)$ & $\mathrm{C} 12-\mathrm{C} 11-\mathrm{H} 11 \mathrm{~A}$ & 109.0 \\
\hline $\mathrm{C} 10-\mathrm{N} 4-\mathrm{C} 7$ & $115.7(3)$ & $\mathrm{S} 2-\mathrm{C} 11-\mathrm{H} 11 \mathrm{~A}$ & 109.0 \\
\hline $\mathrm{C} 2-\mathrm{C} 1-\mathrm{C} 6$ & $122.1(3)$ & $\mathrm{C} 12-\mathrm{C} 11-\mathrm{H} 11 \mathrm{~B}$ & 109.0 \\
\hline $\mathrm{C} 2-\mathrm{C} 1-\mathrm{N} 1$ & $118.5(3)$ & $\mathrm{S} 2-\mathrm{C} 11-\mathrm{H} 11 \mathrm{~B}$ & 109.0 \\
\hline $\mathrm{C} 6-\mathrm{C} 1-\mathrm{N} 1$ & $119.5(3)$ & $\mathrm{H} 11 \mathrm{~A}-\mathrm{C} 11-\mathrm{H} 11 \mathrm{~B}$ & 107.8 \\
\hline $\mathrm{C} 1-\mathrm{C} 2-\mathrm{C} 3$ & $118.2(3)$ & $\mathrm{C} 11-\mathrm{C} 12-\mathrm{H} 12 \mathrm{~A}$ & 109.5 \\
\hline $\mathrm{C} 1-\mathrm{C} 2-\mathrm{H} 2$ & 120.9 & $\mathrm{C} 11-\mathrm{C} 12-\mathrm{H} 12 \mathrm{~B}$ & 109.5 \\
\hline $\mathrm{C} 3-\mathrm{C} 2-\mathrm{H} 2$ & 120.9 & $\mathrm{H} 12 \mathrm{~A}-\mathrm{C} 12-\mathrm{H} 12 \mathrm{~B}$ & 109.5 \\
\hline $\mathrm{C} 4-\mathrm{C} 3-\mathrm{C} 2$ & $120.4(3)$ & $\mathrm{C} 11-\mathrm{C} 12-\mathrm{H} 12 \mathrm{C}$ & 109.5 \\
\hline $\mathrm{C} 4-\mathrm{C} 3-\mathrm{H} 3$ & 119.8 & $\mathrm{H} 12 \mathrm{~A}-\mathrm{C} 12-\mathrm{H} 12 \mathrm{C}$ & 109.5 \\
\hline $\mathrm{C} 2-\mathrm{C} 3-\mathrm{H} 3$ & 119.8 & $\mathrm{H} 12 \mathrm{~B}-\mathrm{C} 12-\mathrm{H} 12 \mathrm{C}$ & 109.5 \\
\hline $\mathrm{C} 3-\mathrm{C} 4-\mathrm{C} 5$ & $121.0(3)$ & & \\
\hline $\mathrm{O} 2-\mathrm{S} 1-\mathrm{N} 2-\mathrm{C} 7$ & $-60.0(3)$ & $\mathrm{C} 4-\mathrm{C} 5-\mathrm{C} 6-\mathrm{C} 1$ & $-0.5(4)$ \\
\hline $\mathrm{O} 1-\mathrm{S} 1-\mathrm{N} 2-\mathrm{C} 7$ & $170.2(2)$ & $\mathrm{C} 2-\mathrm{C} 1-\mathrm{C} 6-\mathrm{C} 5$ & $-1.6(5)$ \\
\hline $\mathrm{C} 4-\mathrm{S} 1-\mathrm{N} 2-\mathrm{C} 7$ & $55.0(3)$ & $\mathrm{N} 1-\mathrm{C} 1-\mathrm{C} 6-\mathrm{C} 5$ & $178.0(3)$ \\
\hline $\mathrm{C} 6-\mathrm{C} 1-\mathrm{C} 2-\mathrm{C} 3$ & $2.4(4)$ & $\mathrm{C} 8-\mathrm{N} 3-\mathrm{C} 7-\mathrm{N} 4$ & $-1.6(5)$ \\
\hline $\mathrm{N} 1-\mathrm{C} 1-\mathrm{C} 2-\mathrm{C} 3$ & $-177.1(3)$ & $\mathrm{C} 8-\mathrm{N} 3-\mathrm{C} 7-\mathrm{N} 2$ & $178.8(3)$ \\
\hline
\end{tabular}




$\begin{array}{ll}\mathrm{C} 1-\mathrm{C} 2-\mathrm{C} 3-\mathrm{C} 4 & -1.3(4) \\ \mathrm{C} 2-\mathrm{C} 3-\mathrm{C} 4-\mathrm{C} 5 & -0.8(5) \\ \mathrm{C} 2-\mathrm{C} 3-\mathrm{C} 4-\mathrm{S} 1 & 176.6(2) \\ \mathrm{O} 2-\mathrm{S} 1-\mathrm{C} 4-\mathrm{C} 3 & -9.6(3) \\ \mathrm{O} 1-\mathrm{S} 1-\mathrm{C} 4-\mathrm{C} 3 & 121.6(2) \\ \mathrm{N} 2-\mathrm{S} 1-\mathrm{C} 4-\mathrm{C} 3 & -127.1(3) \\ \mathrm{O} 2-\mathrm{S} 1-\mathrm{C} 4-\mathrm{C} 5 & 167.8(2) \\ \mathrm{O} 1-\mathrm{S} 1-\mathrm{C} 4-\mathrm{C} 5 & -61.1(3) \\ \mathrm{N} 2-\mathrm{S} 1-\mathrm{C} 4-\mathrm{C} 5 & 50.3(3) \\ \mathrm{C} 3-\mathrm{C} 4-\mathrm{C} 5-\mathrm{C} 6 & 1.7(5) \\ \mathrm{S} 1-\mathrm{C} 4-\mathrm{C} 5-\mathrm{C} 6 & -175.7(2)\end{array}$

$\mathrm{C} 10-\mathrm{N} 4-\mathrm{C} 7-\mathrm{N} 3$

$0.8(4)$

$\mathrm{C} 10-\mathrm{N} 4-\mathrm{C} 7-\mathrm{N} 2$

$-179.6(3)$

$\mathrm{S} 1-\mathrm{N} 2-\mathrm{C} 7-\mathrm{N} 3$

$15.1(4)$

$\mathrm{S} 1-\mathrm{N} 2-\mathrm{C} 7-\mathrm{N} 4$

$-164.5(2)$

$\mathrm{C} 7-\mathrm{N} 3-\mathrm{C} 8-\mathrm{C} 9$

$1.1(5)$

N3-C8-C9-C10

$-0.1(5)$

$\mathrm{C} 7-\mathrm{N} 4-\mathrm{C} 10-\mathrm{C} 9$

$0.5(4)$

$\mathrm{C} 8-\mathrm{C} 9-\mathrm{C} 10-\mathrm{N} 4$

$-0.8(5)$

$64.5(3)$

$-57.7(3)$

$-176.1(3)$

Hydrogen-bond geometry $(\AA, \stackrel{\circ}{)})$

\begin{tabular}{lllll}
\hline$D-\mathrm{H} \cdots A$ & $D-\mathrm{H}$ & $\mathrm{H} \cdots A$ & $D \cdots A$ & $D-\mathrm{H} \cdots A$ \\
\hline $\mathrm{N} 1-\mathrm{H} 1 N \cdots \mathrm{O} 5^{\mathrm{i}}$ & $0.91(1)$ & $2.05(1)$ & $2.929(4)$ & $164(3)$ \\
$\mathrm{N} 1-\mathrm{H} 2 N \cdots \mathrm{O} 4^{4 i}$ & $0.90(1)$ & $2.17(2)$ & $2.980(4)$ & $149(3)$ \\
$\mathrm{N} 1-\mathrm{H} 2 N \cdots \mathrm{O} 3^{\text {iii }}$ & $0.90(1)$ & $2.26(3)$ & $2.705(3)$ & $110(2)$ \\
$\mathrm{N} 1-\mathrm{H} 3 N \cdots \mathrm{O} 5$ & $0.91(1)$ & $1.89(1)$ & $2.801(3)$ & $176(3)$ \\
$\mathrm{N} 2-\mathrm{H} 4 N \cdots \mathrm{N} 4^{\text {iv }}$ & $0.89(3)$ & $1.99(3)$ & $2.882(4)$ & $174(3)$ \\
\hline
\end{tabular}

Symmetry codes: (i) $x,-y+1 / 2, z+1 / 2$; (ii) $x+1, y, z$; (iii) $x+1,-y+1 / 2, z+1 / 2$; (iv) $-x+1,-y+1,-z+1$.

(VIII) 4-[(Pyrimidin-2-yl)sulfamoyl]anilinium 4-hydroxybenzenesulfonate dihydrate

Crystal data

$\mathrm{C}_{10} \mathrm{H}_{11} \mathrm{~N}_{4} \mathrm{O}_{2} \mathrm{~S}^{+} \cdot \mathrm{C}_{6} \mathrm{H}_{5} \mathrm{O}_{4} \mathrm{~S}^{-} \cdot 2 \mathrm{H}_{2} \mathrm{O}$

$M_{r}=460.48$

Monoclinic, $P 2_{1} / n$

Hall symbol: -P 2 yn

$a=18.5872(5) \AA$

$b=5.9733(2) \AA$

$c=18.6850(5) \AA$

$\beta=104.858(3)^{\circ}$

$V=2005.17(10) \AA^{3}$

$Z=4$

Data collection

Oxford Diffraction Xcalibur E diffractometer

Radiation source: fine-focus sealed tube Graphite monochromator

$\omega$ scans

Absorption correction: multi-scan

(CrysAlis PRO; Oxford Diffraction, 2010)

$T_{\min }=0.968, T_{\max }=1.000$

Refinement

Refinement on $F^{2}$

Least-squares matrix: full

$R\left[F^{2}>2 \sigma\left(F^{2}\right)\right]=0.048$

$w R\left(F^{2}\right)=0.113$
$F(000)=960$

$D_{\mathrm{x}}=1.525 \mathrm{Mg} \mathrm{m}^{-3}$

Mo $K \alpha$ radiation, $\lambda=0.71073 \AA$

Cell parameters from 3724 reflections

$\theta=3.3-29.5^{\circ}$

$\mu=0.32 \mathrm{~mm}^{-1}$

$T=123 \mathrm{~K}$

Fragment, light yellow

$0.20 \times 0.12 \times 0.05 \mathrm{~mm}$

9653 measured reflections

4695 independent reflections

3638 reflections with $I>2 \sigma(I)$

$R_{\text {int }}=0.029$

$\theta_{\text {max }}=28.0^{\circ}, \theta_{\min }=3.3^{\circ}$

$h=-24 \rightarrow 24$

$k=-7 \rightarrow 6$

$l=-21 \rightarrow 24$

$S=1.03$

4695 reflections

307 parameters

6 restraints 
Primary atom site location: structure-invariant direct methods

Secondary atom site location: difference Fourier map

Hydrogen site location: inferred from neighbouring sites
$\mathrm{H}$ atoms treated by a mixture of independent and constrained refinement

$w=1 /\left[\sigma^{2}\left(F_{\mathrm{o}}^{2}\right)+(0.0407 P)^{2}+1.8769 P\right]$ where $P=\left(F_{\mathrm{o}}^{2}+2 F_{\mathrm{c}}{ }^{2}\right) / 3$

$(\Delta / \sigma)_{\max }<0.001$

$\Delta \rho_{\max }=0.80$ e $\AA^{-3}$

$\Delta \rho_{\min }=-0.44{\mathrm{e} \AA^{-3}}^{-3}$

\section{Special details}

Experimental. Absorption correction: CrysAlisPro, Oxford Diffraction Ltd., Version 1.171.34.40 (release 27-08-2010 CrysAlis171 .NET) (compiled Aug 27 2010,11:50:40) Empirical absorption correction using spherical harmonics, implemented in SCALE3 ABSPACK scaling algorithm.

Geometry. All s.u.'s (except the s.u. in the dihedral angle between two 1.s. planes) are estimated using the full covariance matrix. The cell s.u.'s are taken into account individually in the estimation of s.u.'s in distances, angles and torsion angles; correlations between s.u.'s in cell parameters are only used when they are defined by crystal symmetry. An approximate (isotropic) treatment of cell s.u.'s is used for estimating s.u.'s involving l.s. planes.

Refinement. Refinement of $F^{2}$ against ALL reflections. The weighted $R$-factor $w R$ and goodness of fit $S$ are based on $F^{2}$, conventional $R$-factors $R$ are based on $F$, with $F$ set to zero for negative $F^{2}$. The threshold expression of $F^{2}>2 \sigma\left(F^{2}\right)$ is used only for calculating $R$-factors $(\mathrm{gt})$ etc. and is not relevant to the choice of reflections for refinement. $R$-factors based on $F^{2}$ are statistically about twice as large as those based on $F$, and $R$ - factors based on ALL data will be even larger.

Fractional atomic coordinates and isotropic or equivalent isotropic displacement parameters $\left(\AA^{2}\right)$

\begin{tabular}{|c|c|c|c|c|}
\hline & $x$ & $y$ & $z$ & $U_{\text {iso }} * / U_{\text {eq }}$ \\
\hline S1 & $0.65276(3)$ & $0.85852(10)$ & 0.14183 & $0.01721(14)$ \\
\hline $\mathrm{S} 2$ & $0.04915(3)$ & $0.16965(10)$ & $0.11296(3)$ & $0.02095(15)$ \\
\hline $\mathrm{O} 1$ & $0.67885(9)$ & $0.6329(3)$ & $0.14790(9)$ & $0.0228(4)$ \\
\hline $\mathrm{O} 2$ & $0.69403(9)$ & $1.0264(3)$ & $0.11393(9)$ & $0.0220(4)$ \\
\hline $\mathrm{O} 1 \mathrm{~W}$ & $0.74103(10)$ & $1.3663(3)$ & $0.49790(10)$ & $0.0294(4)$ \\
\hline $\mathrm{O} 3$ & $0.24507(11)$ & $0.2558(3)$ & $0.41461(10)$ & 0.0307 (4) \\
\hline $\mathrm{O} 2 \mathrm{~W}$ & $0.38462(10)$ & $0.1557(3)$ & $0.45248(10)$ & $0.0253(4)$ \\
\hline $\mathrm{O} 4$ & $0.09613(10)$ & $0.1048(3)$ & $0.06496(9)$ & $0.0245(4)$ \\
\hline $\mathrm{O} 5$ & $0.01741(10)$ & $0.3925(3)$ & $0.09516(10)$ & $0.0296(4)$ \\
\hline O6 & $-0.00589(11)$ & $0.0033(3)$ & $0.11685(10)$ & $0.0361(5)$ \\
\hline N1 & $0.60917(12)$ & $1.1632(4)$ & $0.43098(11)$ & $0.0184(4)$ \\
\hline N2 & $0.56951(11)$ & $0.8768(3)$ & $0.08478(11)$ & $0.0192(4)$ \\
\hline N3 & $0.45276(10)$ & $0.7612(3)$ & $0.02182(10)$ & $0.0184(4)$ \\
\hline N4 & $0.51699(11)$ & $0.5802(3)$ & $0.13473(11)$ & $0.0239(5)$ \\
\hline $\mathrm{C} 1$ & $0.62078(12)$ & $1.0873(4)$ & $0.36057(12)$ & $0.0167(5)$ \\
\hline $\mathrm{C} 2$ & $0.64836(13)$ & $0.8753(4)$ & $0.35556(13)$ & $0.0216(5)$ \\
\hline $\mathrm{H} 2$ & 0.6598 & 0.7791 & 0.3974 & $0.026^{*}$ \\
\hline $\mathrm{C} 3$ & 0.65925 (13) & $0.8042(4)$ & $0.28801(13)$ & $0.0205(5)$ \\
\hline H3 & 0.6779 & 0.6584 & 0.2831 & $0.025^{*}$ \\
\hline $\mathrm{C} 4$ & $0.64263(12)$ & $0.9487(4)$ & $0.22827(12)$ & $0.0153(4)$ \\
\hline $\mathrm{C} 5$ & $0.61737(13)$ & $1.1644(4)$ & $0.23418(13)$ & $0.0201(5)$ \\
\hline H5 & 0.6084 & 1.2637 & 0.1931 & $0.024 *$ \\
\hline C6 & 0.60549 (13) & $1.2328(4)$ & $0.30103(13)$ & $0.0200(5)$ \\
\hline H6 & 0.5870 & 1.3787 & 0.3060 & $0.024 *$ \\
\hline $\mathrm{C} 7$ & $0.51075(12)$ & $0.7306(4)$ & $0.08101(12)$ & $0.0166(5)$ \\
\hline $\mathrm{C} 8$ & $0.39652(13)$ & $0.6169(4)$ & $0.01531(13)$ & $0.0211(5)$ \\
\hline
\end{tabular}




\begin{tabular}{lllll} 
H8 & 0.3544 & 0.6301 & -0.0259 & $0.025^{*}$ \\
C9 & $0.39741(14)$ & $0.4498(4)$ & $0.06624(14)$ & $0.0253(5)$ \\
H9 & 0.3577 & 0.3457 & 0.0605 & $0.030^{*}$ \\
C10 & $0.45878(14)$ & $0.4411(4)$ & $0.12597(14)$ & $0.0270(6)$ \\
H10 & 0.4599 & 0.3308 & 0.1629 & $0.032^{*}$ \\
C11 & $0.10841(13)$ & $0.1923(4)$ & $0.20342(12)$ & $0.0184(5)$ \\
C12 & $0.16396(14)$ & $0.0356(4)$ & $0.22862(14)$ & $0.0249(5)$ \\
H12 & 0.1699 & -0.0849 & 0.1975 & $0.030^{*}$ \\
C13 & $0.21102(14)$ & $0.0540(4)$ & $0.29930(14)$ & $0.0258(5)$ \\
H13 & 0.2494 & -0.0528 & 0.3166 & $0.031^{*}$ \\
C14 & $0.20137(13)$ & $0.2292(4)$ & $0.34422(13)$ & $0.0228(5)$ \\
C15 & $0.14535(15)$ & $0.3837(5)$ & $0.31933(15)$ & $0.0313(6)$ \\
H15 & 0.1387 & 0.5028 & 0.3507 & $0.038^{*}$ \\
C16 & $0.09883(15)$ & $0.3653(4)$ & $0.24877(14)$ & $0.0290(6)$ \\
H16 & 0.0603 & 0.4719 & 0.2316 & $0.035^{*}$ \\
H1N & $0.6528(17)$ & $1.235(5)$ & $0.4588(16)$ & $0.032(8)^{*}$ \\
H2N & $0.5995(17)$ & $1.043(6)$ & $0.4561(17)$ & $0.045(9)^{*}$ \\
H3N & $0.5680(19)$ & $1.260(5)$ & $0.4221(17)$ & $0.046(9)^{*}$ \\
H4N & $0.5636(16)$ & $0.987(5)$ & $0.0532(16)$ & $0.034(8)^{*}$ \\
H1H & $0.292(2)$ & $0.181(7)$ & $0.419(2)$ & $0.088(15)^{*}$ \\
H1W & $0.742(2)$ & $1.482(4)$ & $0.5266(16)$ & $0.071(13)^{*}$ \\
H2W & $0.7630(18)$ & $1.420(5)$ & $0.4655(14)$ & $0.065(12)^{*}$ \\
H3W & $0.4165(14)$ & $0.081(4)$ & $0.4344(17)$ & $0.051(10)^{*}$ \\
H4W & $0.4001(19)$ & $0.295(2)$ & $0.452(2)$ & $0.078(13)^{*}$ \\
& & & & \\
\hline
\end{tabular}

Atomic displacement parameters $\left(\AA^{2}\right)$

\begin{tabular}{lllllll}
\hline & $U^{11}$ & $U^{22}$ & $U^{33}$ & $U^{12}$ & $U^{13}$ & $U^{23}$ \\
\hline S1 & $0.0145(3)$ & $0.0194(3)$ & $0.0167(3)$ & $0.0008(2)$ & $0.0023(2)$ & $-0.0025(2)$ \\
S2 & $0.0188(3)$ & $0.0259(3)$ & $0.0179(3)$ & $-0.0040(2)$ & $0.0044(2)$ & $-0.0013(2)$ \\
O1 & $0.0218(9)$ & $0.0222(9)$ & $0.0228(8)$ & $0.0042(7)$ & $0.0029(7)$ & $-0.0054(7)$ \\
O2 & $0.0187(8)$ & $0.0270(9)$ & $0.0205(8)$ & $-0.0046(7)$ & $0.0056(7)$ & $-0.0020(7)$ \\
O1W & $0.0253(10)$ & $0.0363(11)$ & $0.0280(10)$ & $-0.0046(8)$ & $0.0093(8)$ & $-0.0074(9)$ \\
O3 & $0.0248(10)$ & $0.0409(11)$ & $0.0237(9)$ & $0.0013(9)$ & $0.0016(8)$ & $-0.0093(8)$ \\
O2W & $0.0226(9)$ & $0.0245(10)$ & $0.0273(9)$ & $0.0003(8)$ & $0.0037(7)$ & $0.0032(8)$ \\
O4 & $0.0312(10)$ & $0.0257(9)$ & $0.0182(8)$ & $0.0000(8)$ & $0.0091(7)$ & $0.0001(7)$ \\
O5 & $0.0229(9)$ & $0.0340(10)$ & $0.0305(9)$ & $0.0065(8)$ & $0.0041(8)$ & $0.0040(8)$ \\
O6 & $0.0373(11)$ & $0.0453(12)$ & $0.0258(9)$ & $-0.0218(9)$ & $0.0082(8)$ & $-0.0038(9)$ \\
N1 & $0.0186(10)$ & $0.0190(10)$ & $0.0185(10)$ & $-0.0004(9)$ & $0.0062(8)$ & $-0.0008(9)$ \\
N2 & $0.0166(10)$ & $0.0211(10)$ & $0.0169(9)$ & $-0.0022(8)$ & $-0.0012(8)$ & $0.0041(8)$ \\
N3 & $0.0156(9)$ & $0.0193(10)$ & $0.0193(9)$ & $-0.0011(8)$ & $0.0027(8)$ & $-0.0014(8)$ \\
N4 & $0.0217(11)$ & $0.0232(10)$ & $0.0255(11)$ & $-0.0026(9)$ & $0.0035(9)$ & $0.0072(9)$ \\
C1 & $0.0127(10)$ & $0.0186(11)$ & $0.0180(11)$ & $-0.0005(9)$ & $0.0027(8)$ & $-0.0024(9)$ \\
C2 & $0.0248(12)$ & $0.0208(12)$ & $0.0185(11)$ & $0.0048(10)$ & $0.0041(9)$ & $0.0051(10)$ \\
C3 & $0.0238(12)$ & $0.0149(11)$ & $0.0224(12)$ & $0.0048(9)$ & $0.0050(10)$ & $0.0000(9)$ \\
C4 & $0.0121(10)$ & $0.0172(11)$ & $0.0159(10)$ & $0.0004(9)$ & $0.0021(8)$ & $-0.0007(9)$ \\
C5 & $0.0237(12)$ & $0.0186(11)$ & $0.0180(11)$ & $0.0045(10)$ & $0.0053(9)$ & $0.0035(9)$ \\
C6 & $0.0262(13)$ & $0.0135(11)$ & $0.0214(11)$ & $0.0048(9)$ & $0.0079(10)$ & $0.0003(9)$
\end{tabular}




\begin{tabular}{lllllll} 
C7 & $0.0161(11)$ & $0.0159(11)$ & $0.0179(11)$ & $0.0010(9)$ & $0.0043(9)$ & $-0.0017(9)$ \\
C8 & $0.0175(11)$ & $0.0232(12)$ & $0.0218(12)$ & $-0.0007(10)$ & $0.0038(9)$ & $-0.0037(10)$ \\
C9 & $0.0202(12)$ & $0.0225(12)$ & $0.0339(14)$ & $-0.0061(10)$ & $0.0079(10)$ & $0.0011(11)$ \\
C10 & $0.0249(13)$ & $0.0244(13)$ & $0.0314(13)$ & $-0.0019(11)$ & $0.0070(11)$ & $0.0087(11)$ \\
C11 & $0.0169(11)$ & $0.0211(12)$ & $0.0174(11)$ & $-0.0024(9)$ & $0.0050(9)$ & $-0.0028(9)$ \\
C12 & $0.0254(13)$ & $0.0248(13)$ & $0.0252(12)$ & $0.0035(11)$ & $0.0075(10)$ & $-0.0079(10)$ \\
C13 & $0.0207(12)$ & $0.0280(13)$ & $0.0277(13)$ & $0.0066(11)$ & $0.0042(10)$ & $0.0002(11)$ \\
C14 & $0.0198(12)$ & $0.0286(13)$ & $0.0194(11)$ & $-0.0058(10)$ & $0.0042(9)$ & $-0.0064(10)$ \\
C15 & $0.0320(15)$ & $0.0299(14)$ & $0.0301(14)$ & $0.0051(12)$ & $0.0046(11)$ & $-0.0141(11)$ \\
C16 & $0.0292(14)$ & $0.0272(14)$ & $0.0280(13)$ & $0.0079(11)$ & $0.0026(11)$ & $-0.0056(11)$ \\
\hline
\end{tabular}

Geometric parameters $\left(\AA,{ }^{\circ}\right)$

\begin{tabular}{|c|c|c|c|}
\hline $\mathrm{S} 1-\mathrm{O} 1$ & $1.4271(17)$ & $\mathrm{C} 1-\mathrm{C} 6$ & $1.383(3)$ \\
\hline $\mathrm{S} 1-\mathrm{O} 2$ & $1.4390(17)$ & $\mathrm{C} 2-\mathrm{C} 3$ & $1.395(3)$ \\
\hline $\mathrm{S} 1-\mathrm{N} 2$ & $1.6422(19)$ & $\mathrm{C} 2-\mathrm{H} 2$ & 0.9500 \\
\hline $\mathrm{S} 1-\mathrm{C} 4$ & $1.758(2)$ & $\mathrm{C} 3-\mathrm{C} 4$ & $1.382(3)$ \\
\hline $\mathrm{S} 2-\mathrm{O} 6$ & $1.4413(19)$ & $\mathrm{C} 3-\mathrm{H} 3$ & 0.9500 \\
\hline $\mathrm{S} 2-\mathrm{O} 4$ & $1.4557(18)$ & $\mathrm{C} 4-\mathrm{C} 5$ & $1.386(3)$ \\
\hline $\mathrm{S} 2-\mathrm{O} 5$ & 1.4597 (19) & $\mathrm{C} 5-\mathrm{C} 6$ & $1.385(3)$ \\
\hline $\mathrm{S} 2-\mathrm{C} 11$ & $1.770(2)$ & $\mathrm{C} 5-\mathrm{H} 5$ & 0.9500 \\
\hline $\mathrm{O} 1 \mathrm{~W}-\mathrm{H} 1 \mathrm{~W}$ & $0.873(10)$ & $\mathrm{C} 6-\mathrm{H} 6$ & 0.9500 \\
\hline $\mathrm{O} 1 \mathrm{~W}-\mathrm{H} 2 \mathrm{~W}$ & $0.874(10)$ & $\mathrm{C} 8-\mathrm{C} 9$ & $1.376(3)$ \\
\hline $\mathrm{O} 3-\mathrm{C} 14$ & $1.366(3)$ & $\mathrm{C} 8-\mathrm{H} 8$ & 0.9500 \\
\hline $\mathrm{O} 3-\mathrm{H} 1 \mathrm{H}$ & $0.96(4)$ & $\mathrm{C} 9-\mathrm{C} 10$ & $1.377(3)$ \\
\hline $\mathrm{O} 2 \mathrm{~W}-\mathrm{H} 3 \mathrm{~W}$ & $0.875(10)$ & $\mathrm{C} 9-\mathrm{H} 9$ & 0.9500 \\
\hline $\mathrm{O} 2 \mathrm{~W}-\mathrm{H} 4 \mathrm{~W}$ & $0.880(10)$ & $\mathrm{C} 10-\mathrm{H} 10$ & 0.9500 \\
\hline $\mathrm{N} 1-\mathrm{C} 1$ & $1.459(3)$ & $\mathrm{C} 11-\mathrm{C} 16$ & $1.376(3)$ \\
\hline $\mathrm{N} 1-\mathrm{H} 1 \mathrm{~N}$ & $0.95(3)$ & $\mathrm{C} 11-\mathrm{C} 12$ & $1.384(3)$ \\
\hline $\mathrm{N} 1-\mathrm{H} 2 \mathrm{~N}$ & $0.90(3)$ & $\mathrm{C} 12-\mathrm{C} 13$ & $1.389(3)$ \\
\hline $\mathrm{N} 1-\mathrm{H} 3 \mathrm{~N}$ & $0.94(3)$ & $\mathrm{C} 12-\mathrm{H} 12$ & 0.9500 \\
\hline $\mathrm{N} 2-\mathrm{C} 7$ & $1.386(3)$ & $\mathrm{C} 13-\mathrm{C} 14$ & $1.382(4)$ \\
\hline $\mathrm{N} 2-\mathrm{H} 4 \mathrm{~N}$ & $0.87(3)$ & $\mathrm{C} 13-\mathrm{H} 13$ & 0.9500 \\
\hline $\mathrm{N} 3-\mathrm{C} 8$ & $1.336(3)$ & $\mathrm{C} 14-\mathrm{C} 15$ & $1.380(4)$ \\
\hline $\mathrm{N} 3-\mathrm{C} 7$ & $1.344(3)$ & $\mathrm{C} 15-\mathrm{C} 16$ & $1.383(3)$ \\
\hline $\mathrm{N} 4-\mathrm{C} 7$ & $1.330(3)$ & C15-H15 & 0.9500 \\
\hline $\mathrm{N} 4-\mathrm{C} 10$ & $1.340(3)$ & $\mathrm{C} 16-\mathrm{H} 16$ & 0.9500 \\
\hline $\mathrm{C} 1-\mathrm{C} 2$ & $1.378(3)$ & & \\
\hline $\mathrm{O} 1-\mathrm{S} 1-\mathrm{O} 2$ & $119.01(10)$ & $\mathrm{C} 5-\mathrm{C} 4-\mathrm{S} 1$ & $118.46(17)$ \\
\hline $\mathrm{O} 1-\mathrm{S} 1-\mathrm{N} 2$ & $111.03(10)$ & $\mathrm{C} 6-\mathrm{C} 5-\mathrm{C} 4$ & $118.8(2)$ \\
\hline $\mathrm{O} 2-\mathrm{S} 1-\mathrm{N} 2$ & $102.68(10)$ & $\mathrm{C} 6-\mathrm{C} 5-\mathrm{H} 5$ & 120.6 \\
\hline $\mathrm{O} 1-\mathrm{S} 1-\mathrm{C} 4$ & $109.32(10)$ & $\mathrm{C} 4-\mathrm{C} 5-\mathrm{H} 5$ & 120.6 \\
\hline $\mathrm{O} 2-\mathrm{S} 1-\mathrm{C} 4$ & $108.18(10)$ & $\mathrm{C} 1-\mathrm{C} 6-\mathrm{C} 5$ & $119.5(2)$ \\
\hline $\mathrm{N} 2-\mathrm{S} 1-\mathrm{C} 4$ & $105.72(10)$ & $\mathrm{C} 1-\mathrm{C} 6-\mathrm{H} 6$ & 120.2 \\
\hline $\mathrm{O} 6-\mathrm{S} 2-\mathrm{O} 4$ & $113.15(11)$ & $\mathrm{C} 5-\mathrm{C} 6-\mathrm{H} 6$ & 120.2 \\
\hline $\mathrm{O} 6-\mathrm{S} 2-\mathrm{O} 5$ & $112.94(12)$ & $\mathrm{N} 4-\mathrm{C} 7-\mathrm{N} 3$ & $127.5(2)$ \\
\hline $\mathrm{O} 4-\mathrm{S} 2-\mathrm{O} 5$ & $111.94(11)$ & $\mathrm{N} 4-\mathrm{C} 7-\mathrm{N} 2$ & $118.2(2)$ \\
\hline
\end{tabular}




\begin{tabular}{|c|c|c|c|}
\hline $\mathrm{O} 6-\mathrm{S} 2-\mathrm{C} 11$ & $106.29(11)$ & $\mathrm{N} 3-\mathrm{C} 7-\mathrm{N} 2$ & $114.3(2)$ \\
\hline $\mathrm{O} 4-\mathrm{S} 2-\mathrm{C} 11$ & $106.50(11)$ & $\mathrm{N} 3-\mathrm{C} 8-\mathrm{C} 9$ & $122.3(2)$ \\
\hline $\mathrm{O} 5-\mathrm{S} 2-\mathrm{C} 11$ & $105.31(11)$ & $\mathrm{N} 3-\mathrm{C} 8-\mathrm{H} 8$ & 118.8 \\
\hline $\mathrm{H} 1 \mathrm{~W}-\mathrm{O} 1 \mathrm{~W}-\mathrm{H} 2 \mathrm{~W}$ & $101(2)$ & $\mathrm{C} 9-\mathrm{C} 8-\mathrm{H} 8$ & 118.8 \\
\hline $\mathrm{C} 14-\mathrm{O} 3-\mathrm{H} 1 \mathrm{H}$ & $109(3)$ & $\mathrm{C} 8-\mathrm{C} 9-\mathrm{C} 10$ & $116.6(2)$ \\
\hline $\mathrm{H} 3 \mathrm{~W}-\mathrm{O} 2 \mathrm{~W}-\mathrm{H} 4 \mathrm{~W}$ & $103(2)$ & $\mathrm{C} 8-\mathrm{C} 9-\mathrm{H} 9$ & 121.7 \\
\hline $\mathrm{C} 1-\mathrm{N} 1-\mathrm{H} 1 \mathrm{~N}$ & 109.4 (17) & $\mathrm{C} 10-\mathrm{C} 9-\mathrm{H} 9$ & 121.7 \\
\hline $\mathrm{C} 1-\mathrm{N} 1-\mathrm{H} 2 \mathrm{~N}$ & $109(2)$ & $\mathrm{N} 4-\mathrm{C} 10-\mathrm{C} 9$ & $123.4(2)$ \\
\hline $\mathrm{H} 1 \mathrm{~N}-\mathrm{N} 1-\mathrm{H} 2 \mathrm{~N}$ & $109(3)$ & $\mathrm{N} 4-\mathrm{C} 10-\mathrm{H} 10$ & 118.3 \\
\hline $\mathrm{C} 1-\mathrm{N} 1-\mathrm{H} 3 \mathrm{~N}$ & 109.5 (19) & $\mathrm{C} 9-\mathrm{C} 10-\mathrm{H} 10$ & 118.3 \\
\hline $\mathrm{H} 1 \mathrm{~N}-\mathrm{N} 1-\mathrm{H} 3 \mathrm{~N}$ & $111(3)$ & $\mathrm{C} 16-\mathrm{C} 11-\mathrm{C} 12$ & $120.1(2)$ \\
\hline $\mathrm{H} 2 \mathrm{~N}-\mathrm{N} 1-\mathrm{H} 3 \mathrm{~N}$ & $109(3)$ & $\mathrm{C} 16-\mathrm{C} 11-\mathrm{S} 2$ & $119.87(19)$ \\
\hline $\mathrm{C} 7-\mathrm{N} 2-\mathrm{S} 1$ & $126.17(17)$ & $\mathrm{C} 12-\mathrm{C} 11-\mathrm{S} 2$ & $120.04(18)$ \\
\hline $\mathrm{C} 7-\mathrm{N} 2-\mathrm{H} 4 \mathrm{~N}$ & $118.7(19)$ & $\mathrm{C} 11-\mathrm{C} 12-\mathrm{C} 13$ & $120.2(2)$ \\
\hline $\mathrm{S} 1-\mathrm{N} 2-\mathrm{H} 4 \mathrm{~N}$ & $115(2)$ & $\mathrm{C} 11-\mathrm{C} 12-\mathrm{H} 12$ & 119.9 \\
\hline $\mathrm{C} 8-\mathrm{N} 3-\mathrm{C} 7$ & $115.5(2)$ & $\mathrm{C} 13-\mathrm{C} 12-\mathrm{H} 12$ & 119.9 \\
\hline $\mathrm{C} 7-\mathrm{N} 4-\mathrm{C} 10$ & $114.5(2)$ & $\mathrm{C} 14-\mathrm{C} 13-\mathrm{C} 12$ & $119.3(2)$ \\
\hline $\mathrm{C} 2-\mathrm{C} 1-\mathrm{C} 6$ & $121.7(2)$ & $\mathrm{C} 14-\mathrm{C} 13-\mathrm{H} 13$ & 120.4 \\
\hline $\mathrm{C} 2-\mathrm{C} 1-\mathrm{N} 1$ & $119.4(2)$ & $\mathrm{C} 12-\mathrm{C} 13-\mathrm{H} 13$ & 120.4 \\
\hline $\mathrm{C} 6-\mathrm{C} 1-\mathrm{N} 1$ & $118.9(2)$ & $\mathrm{O} 3-\mathrm{C} 14-\mathrm{C} 15$ & $117.2(2)$ \\
\hline $\mathrm{C} 1-\mathrm{C} 2-\mathrm{C} 3$ & $118.9(2)$ & $\mathrm{O} 3-\mathrm{C} 14-\mathrm{C} 13$ & $122.3(2)$ \\
\hline $\mathrm{C} 1-\mathrm{C} 2-\mathrm{H} 2$ & 120.5 & $\mathrm{C} 15-\mathrm{C} 14-\mathrm{C} 13$ & $120.4(2)$ \\
\hline $\mathrm{C} 3-\mathrm{C} 2-\mathrm{H} 2$ & 120.5 & $\mathrm{C} 14-\mathrm{C} 15-\mathrm{C} 16$ & $120.1(2)$ \\
\hline $\mathrm{C} 4-\mathrm{C} 3-\mathrm{C} 2$ & $119.1(2)$ & $\mathrm{C} 14-\mathrm{C} 15-\mathrm{H} 15$ & 120.0 \\
\hline $\mathrm{C} 4-\mathrm{C} 3-\mathrm{H} 3$ & 120.4 & $\mathrm{C} 16-\mathrm{C} 15-\mathrm{H} 15$ & 120.0 \\
\hline $\mathrm{C} 2-\mathrm{C} 3-\mathrm{H} 3$ & 120.4 & $\mathrm{C} 11-\mathrm{C} 16-\mathrm{C} 15$ & $119.9(2)$ \\
\hline $\mathrm{C} 3-\mathrm{C} 4-\mathrm{C} 5$ & $121.8(2)$ & $\mathrm{C} 11-\mathrm{C} 16-\mathrm{H} 16$ & 120.1 \\
\hline $\mathrm{C} 3-\mathrm{C} 4-\mathrm{S} 1$ & $119.76(17)$ & $\mathrm{C} 15-\mathrm{C} 16-\mathrm{H} 16$ & 120.1 \\
\hline $\mathrm{O} 1-\mathrm{S} 1-\mathrm{N} 2-\mathrm{C} 7$ & $42.5(2)$ & $\mathrm{S} 1-\mathrm{N} 2-\mathrm{C} 7-\mathrm{N} 4$ & $10.8(3)$ \\
\hline $\mathrm{O} 2-\mathrm{S} 1-\mathrm{N} 2-\mathrm{C} 7$ & $170.77(19)$ & $\mathrm{S} 1-\mathrm{N} 2-\mathrm{C} 7-\mathrm{N} 3$ & $-169.56(17)$ \\
\hline $\mathrm{C} 4-\mathrm{S} 1-\mathrm{N} 2-\mathrm{C} 7$ & $-75.9(2)$ & $\mathrm{C} 7-\mathrm{N} 3-\mathrm{C} 8-\mathrm{C} 9$ & $0.8(3)$ \\
\hline $\mathrm{C} 6-\mathrm{C} 1-\mathrm{C} 2-\mathrm{C} 3$ & $-1.7(4)$ & $\mathrm{N} 3-\mathrm{C} 8-\mathrm{C} 9-\mathrm{C} 10$ & $1.6(4)$ \\
\hline $\mathrm{N} 1-\mathrm{C} 1-\mathrm{C} 2-\mathrm{C} 3$ & $180.0(2)$ & $\mathrm{C} 7-\mathrm{N} 4-\mathrm{C} 10-\mathrm{C} 9$ & $0.6(4)$ \\
\hline $\mathrm{C} 1-\mathrm{C} 2-\mathrm{C} 3-\mathrm{C} 4$ & $0.5(4)$ & $\mathrm{C} 8-\mathrm{C} 9-\mathrm{C} 10-\mathrm{N} 4$ & $-2.3(4)$ \\
\hline $\mathrm{C} 2-\mathrm{C} 3-\mathrm{C} 4-\mathrm{C} 5$ & $1.8(4)$ & $\mathrm{O} 6-\mathrm{S} 2-\mathrm{C} 11-\mathrm{C} 16$ & $-99.8(2)$ \\
\hline $\mathrm{C} 2-\mathrm{C} 3-\mathrm{C} 4-\mathrm{S} 1$ & $-177.68(18)$ & $\mathrm{O} 4-\mathrm{S} 2-\mathrm{C} 11-\mathrm{C} 16$ & $139.3(2)$ \\
\hline $\mathrm{O} 1-\mathrm{S} 1-\mathrm{C} 4-\mathrm{C} 3$ & $-0.2(2)$ & $\mathrm{O} 5-\mathrm{S} 2-\mathrm{C} 11-\mathrm{C} 16$ & $20.3(2)$ \\
\hline $\mathrm{O} 2-\mathrm{S} 1-\mathrm{C} 4-\mathrm{C} 3$ & $-131.21(19)$ & $\mathrm{O} 6-\mathrm{S} 2-\mathrm{C} 11-\mathrm{C} 12$ & $79.8(2)$ \\
\hline $\mathrm{N} 2-\mathrm{S} 1-\mathrm{C} 4-\mathrm{C} 3$ & $119.37(19)$ & $\mathrm{O} 4-\mathrm{S} 2-\mathrm{C} 11-\mathrm{C} 12$ & $-41.1(2)$ \\
\hline $\mathrm{O} 1-\mathrm{S} 1-\mathrm{C} 4-\mathrm{C} 5$ & $-179.72(17)$ & $\mathrm{O} 5-\mathrm{S} 2-\mathrm{C} 11-\mathrm{C} 12$ & $-160.1(2)$ \\
\hline $\mathrm{O} 2-\mathrm{S} 1-\mathrm{C} 4-\mathrm{C} 5$ & $49.3(2)$ & $\mathrm{C} 16-\mathrm{C} 11-\mathrm{C} 12-\mathrm{C} 13$ & $-1.0(4)$ \\
\hline $\mathrm{N} 2-\mathrm{S} 1-\mathrm{C} 4-\mathrm{C} 5$ & $-60.1(2)$ & $\mathrm{S} 2-\mathrm{C} 11-\mathrm{C} 12-\mathrm{C} 13$ & $179.4(2)$ \\
\hline $\mathrm{C} 3-\mathrm{C} 4-\mathrm{C} 5-\mathrm{C} 6$ & $-2.9(4)$ & $\mathrm{C} 11-\mathrm{C} 12-\mathrm{C} 13-\mathrm{C} 14$ & $0.5(4)$ \\
\hline $\mathrm{S} 1-\mathrm{C} 4-\mathrm{C} 5-\mathrm{C} 6$ & $176.64(18)$ & $\mathrm{C} 12-\mathrm{C} 13-\mathrm{C} 14-\mathrm{O} 3$ & $179.2(2)$ \\
\hline $\mathrm{C} 2-\mathrm{C} 1-\mathrm{C} 6-\mathrm{C} 5$ & $0.6(4)$ & $\mathrm{C} 12-\mathrm{C} 13-\mathrm{C} 14-\mathrm{C} 15$ & $0.3(4)$ \\
\hline $\mathrm{N} 1-\mathrm{C} 1-\mathrm{C} 6-\mathrm{C} 5$ & $179.0(2)$ & $\mathrm{O} 3-\mathrm{C} 14-\mathrm{C} 15-\mathrm{C} 16$ & $-179.6(3)$ \\
\hline $\mathrm{C} 4-\mathrm{C} 5-\mathrm{C} 6-\mathrm{C} 1$ & $1.6(4)$ & $\mathrm{C} 13-\mathrm{C} 14-\mathrm{C} 15-\mathrm{C} 16$ & $-0.7(4)$ \\
\hline
\end{tabular}




\section{supporting information}

$\begin{array}{llll}\mathrm{C} 10-\mathrm{N} 4-\mathrm{C} 7-\mathrm{N} 3 & 2.2(4) & \mathrm{C} 12-\mathrm{C} 11-\mathrm{C} 16-\mathrm{C} 15 & 0.7 \text { (4) } \\ \mathrm{C} 10-\mathrm{N} 4-\mathrm{C} 7-\mathrm{N} 2 & -178.2(2) & \mathrm{S} 2-\mathrm{C} 11-\mathrm{C} 16-\mathrm{C} 15 & -179.7(2) \\ \mathrm{C} 8-\mathrm{N} 3-\mathrm{C} 7-\mathrm{N} 4 & -2.9(4) & \mathrm{C} 14-\mathrm{C} 15-\mathrm{C} 16-\mathrm{C} 11 & 0.1(4) \\ \mathrm{C} 8-\mathrm{N} 3-\mathrm{C} 7-\mathrm{N} 2 & 177.5(2) & & \end{array}$

Hydrogen-bond geometry $\left(\AA,{ }^{\circ}\right)$

\begin{tabular}{|c|c|c|c|c|}
\hline$D-\mathrm{H} \cdots A$ & $D-\mathrm{H}$ & $\mathrm{H} \cdots A$ & $D^{\cdots} A$ & $D-\mathrm{H} \cdots A$ \\
\hline $\mathrm{N} 1-\mathrm{H} 1 N \cdots \mathrm{O} 1 W$ & $0.95(3)$ & $1.80(3)$ & $2.733(3)$ & $170(3)$ \\
\hline $\mathrm{N} 1-\mathrm{H} 2 N^{\cdots} \cdot \mathrm{O} 2 W^{\mathrm{i}}$ & $0.90(3)$ & $2.04(3)$ & $2.873(3)$ & $154(3)$ \\
\hline $\mathrm{N} 1-\mathrm{H} 2 N \cdots \mathrm{O} 5^{\mathrm{ii}}$ & $0.90(3)$ & $2.32(3)$ & $2.793(3)$ & $113(2)$ \\
\hline $\mathrm{N} 1-\mathrm{H} 3 N \cdots \mathrm{O}^{6 i i}$ & $0.94(3)$ & $1.88(3)$ & $2.780(3)$ & $159(3)$ \\
\hline $\mathrm{N} 2-\mathrm{H} 4 N \cdots \mathrm{N} 3^{\text {iv }}$ & $0.87(3)$ & $2.03(3)$ & $2.897(3)$ & $179(3)$ \\
\hline $\mathrm{O} 3-\mathrm{H} 1 H \cdots \mathrm{O} 2 W$ & $0.96(4)$ & $1.69(4)$ & $2.578(3)$ & $153(4)$ \\
\hline $\mathrm{O} 1 W-\mathrm{H} 1 W \cdots \mathrm{O} 3^{v}$ & $0.87(1)$ & $1.89(1)$ & $2.760(3)$ & $173(3)$ \\
\hline $\mathrm{O} 1 W-\mathrm{H} 2 W \cdots \mathrm{O} 2^{\mathrm{vi}}$ & $0.87(1)$ & $1.96(1)$ & $2.834(3)$ & $175(3)$ \\
\hline $\mathrm{O} 2 W-\mathrm{H} 3 W \cdots \mathrm{O} 5^{\mathrm{vii}}$ & $0.88(1)$ & $1.85(1)$ & $2.722(3)$ & $172(3)$ \\
\hline $\mathrm{O} 2 W-\mathrm{H} 4 W \cdots \mathrm{O} 4^{\mathrm{ii}}$ & $0.88(1)$ & $1.88(1)$ & $2.737(2)$ & $163(4)$ \\
\hline
\end{tabular}

Symmetry codes: (i) $-x+1,-y+1,-z+1$; (ii) $-x+1 / 2, y+1 / 2,-z+1 / 2$; (iii) $-x+1 / 2, y+3 / 2,-z+1 / 2$; (iv) $-x+1,-y+2,-z$; (v) $-x+1,-y+2,-z+1$; (vi) $-x+3 / 2$, $y+1 / 2,-z+1 / 2 ;$ (vii) $-x+1 / 2, y-1 / 2,-z+1 / 2$. 